\title{
Autocrine vitamin D signaling switches off pro-inflammatory programs of $T_{H} 1$ cells
}

\author{
Daniel Chauss ${ }^{1,20}$, Tilo Freiwald ${ }^{1,2,20}$, Reuben McGregor ${ }^{1,3,20}$, Bingyu Yan ${ }^{4,20}$, Luopin Wang ${ }^{5,20}$, \\ Estefania Nova-Lamperti ${ }^{6}{ }^{6}$, Dhaneshwar Kumar ${ }^{1,5}$, Zonghao Zhang7, Heather Teague ${ }^{8}$, Erin E. West ${ }^{9}$, \\ Kevin M. Vannella ${ }^{10,11}$, Marcos J. Ramos-Benitez ${ }^{10,11}$, Jack Bibby ${ }^{9}$, Audrey Kelly ${ }^{12}$, Amna Malik ${ }^{13}$, \\ Alexandra F. Freeman ${ }^{14}$, Daniella M. Schwartz ${ }^{15}$, Didier Portilla ${ }^{1,16}$, Daniel S. Chertow ${ }^{10,11}$, Susan John ${ }^{12}$, \\ Paul Lavender ${ }^{12}$, Claudia Kemper ${ }^{9}{ }^{9,17}$, Giovanna Lombardi ${ }^{12}{ }^{12}$, Nehal N. Mehta ${ }^{8}$, Nichola Cooper ${ }^{13}$, \\ Michail S. Lionakis ${ }^{18}{ }^{18}$, Arian Laurence ${ }^{19}$, Majid Kazemian $\mathbb{1}^{4,5,21 凶}$ and Behdad Afzali $\mathbb{1}^{1,21 凶}$
}

The molecular mechanisms governing orderly shutdown and retraction of $\mathrm{CD4}^{+}$type 1 helper $T\left(T_{H} 1\right)$ cell responses remain poorly understood. Here we show that complement triggers contraction of $T_{H} 1$ responses by inducing intrinsic expression of the vitamin D (VitD) receptor and the VitD-activating enzyme CYP27B1, permitting $T$ cells to both activate and respond to VitD. VitD then initiated the transition from pro-inflammatory interferon- $\gamma^{+} T_{H} 1$ cells to suppressive interleukin-10 ${ }^{+}$cells. This process was primed by dynamic changes in the epigenetic landscape of $\mathrm{CD4}^{+} \mathrm{T}$ cells, generating super-enhancers and recruiting several transcription factors, notably c-JUN, STAT3 and BACH2, which together with VitD receptor shaped the transcriptional response to VitD. Accordingly, VitD did not induce interleukin-10 expression in cells with dysfunctional BACH2 or STAT3. Bronchoalveolar lavage fluid $\mathrm{CD4}^{+} \mathrm{T}$ cells of patients with COVID-19 were $\mathrm{T}_{\mathrm{H}}$ 1-skewed and showed de-repression of genes downregulated by VitD, from either lack of substrate (VitD deficiency) and/or abnormal regulation of this system.

A substantial number of patients with COVID-19 develop severe and life-threatening hyper-inflammation and acute respiratory distress syndrome (ARDS). Mortality from severe COVID-19 remains high, in part due to the limited range of specific immunomodulatory therapies available. Survivors, and those with milder disease, may lose significant tissue function from persistent inflammation and fibrosis, causing chronic lung disease. The efficacy of dexamethasone in reducing mortality indicates the importance of inflammation to disease severity ${ }^{1}$. Improved understanding of the basic mechanisms of COVID-19 will aid rational drug design to reduce morbidity and mortality.

Pro-inflammatory immune responses are necessary for pathogen clearance but cause severe tissue damage if not shut down in a timely manner ${ }^{2}$. The complement system is instrumental in pathogen clearance via recruitment and activation of immune cells ${ }^{3}$. In brief, complement $(\mathrm{C}) 3$, a pro-enzyme, is activated in response to pathogen- or danger-sensing (the lectin pathway), immune complexes (classical pathway) or altered self (alternative pathway) to generate active $\mathrm{C} 3 \mathrm{a}$ and $\mathrm{C} 3 \mathrm{~b}$ fragments, which recruit and activate immune cells and instigate activation of downstream complement components ${ }^{4}$. Complement activation is a pathophysiological feature of ARDS of many etiologies ${ }^{5}$ and mediates acute lung injury driven by respiratory viruses ${ }^{6}$. Circulating concentrations of activated complement fragments are high in COVID-19, correlate with severity and are independently associated with mortality ${ }^{7,8}$. Polymorphisms in complement regulators are, likewise, risk factors for poor outcomes 9 . Animal models of other beta-coronaviruses have indicated complement as part of a pathologic signature of

IImmunoregulation Section, Kidney Diseases Branch, National Institute of Diabetes and Digestive and Kidney Diseases (NIDDK), National Institutes of Health, Bethesda, MD, USA. ${ }^{2}$ Medic Clinic III, Department of Nephrology, University Hospital Frankfurt, Goethe-University, Frankfurt, Germany. ${ }^{3}$ Department of Molecular Medicine and Pathology, School of Medical Sciences, The University of Auckland, Auckland, New Zealand. ${ }^{4}$ Department of Biochemistry, Purdue University, West Lafayette, IN, USA. ${ }^{5}$ Department of Computer Science, Purdue University, West Lafayette, IN, USA. ${ }^{6}$ Molecular and Translational Immunology Laboratory, Department of Clinical Biochemistry and Immunology, Faculty of Pharmacy, Universidad de Concepcion, Concepcion, Chile. ${ }^{7}$ Department of Agricultural and Biological Engineering, Purdue University, West Lafayette, IN, USA. ${ }^{8}$ Laboratory of Inflammation \& Cardiometabolic Diseases, Cardiovascular Branch, National Heart, Lung, and Blood Institute (NHLBI), National Institutes of Health, Bethesda, MD, USA. ${ }^{9}$ Complement and Inflammation Research Section, National Heart, Lung, and Blood Institute (NHLBI), National Institutes of Health, Bethesda, MD, USA. ${ }^{10}$ Laboratory of Immunoregulation, National Institute of Allergy and Infectious Diseases (NIAID), National Institutes of Health, Bethesda, MD, USA. "'Emerging Pathogens Section, Critical Care Medicine Department, Clinical Center, National Institutes of Health, Bethesda, MD, USA. ${ }^{12}$ Peter Gorer Department of Immunobiology, School of Immunology and Microbial Sciences, Faculty of Life Sciences and Medicine, King's College London, London, UK. ${ }^{13}$ Department of Medicine, Imperial College London, London, UK. ${ }^{14}$ Laboratory of Clinical Immunology \& Microbiology, National Institute of Allergy and Infectious Diseases (NIAID), National Institutes of Health, Bethesda, MD, USA. ${ }^{15}$ Laboratory of Allergic Diseases, National Institute of Allergy and Infectious Diseases (NIAID), National Institutes of Health, Bethesda, MD, USA. ${ }^{16}$ Division of Nephrology and the Center for Immunity, Inflammation and Regenerative Medicine, University of Virginia, Charlottesville, VA, USA. ${ }^{17}$ Institute for Systemic Inflammation Research, University of Lübeck, Lübeck, Germany. ${ }^{18} \mathrm{Fungal}$ Pathogenesis Section, Laboratory of Clinical Immunology and Microbiology, National Institute of Allergy and Infectious Diseases (NIAID), National Institutes of Health, Bethesda, MD, USA. ${ }^{19}$ Nuffield Department of Medicine, University of Oxford, Oxford, UK. ${ }^{20}$ These authors contributed equally: Daniel Chauss, Tilo Freiwald, Reuben McGregor, Bingyu Yan, Luopin Wang. ${ }^{21}$ These authors jointly supervised this work: Majid Kazemian, Behdad Afzali. $\bowtie$ e-mail: kazemian@purdue.edu; behdad.afzali@nih.gov 
a

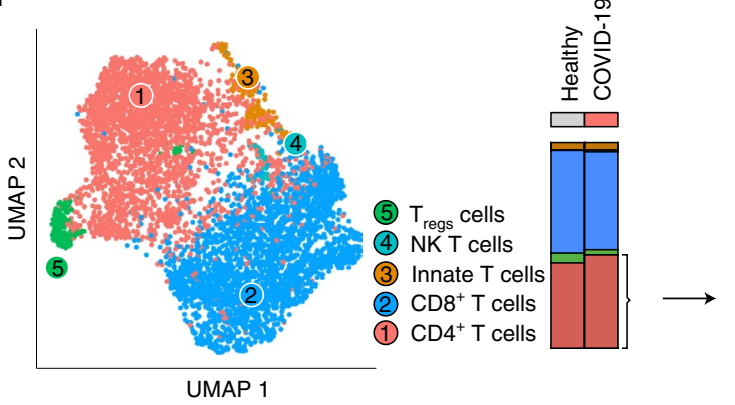

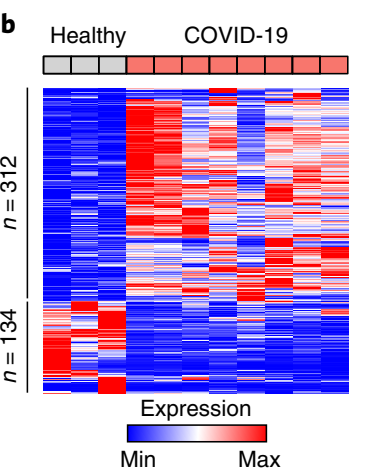

C

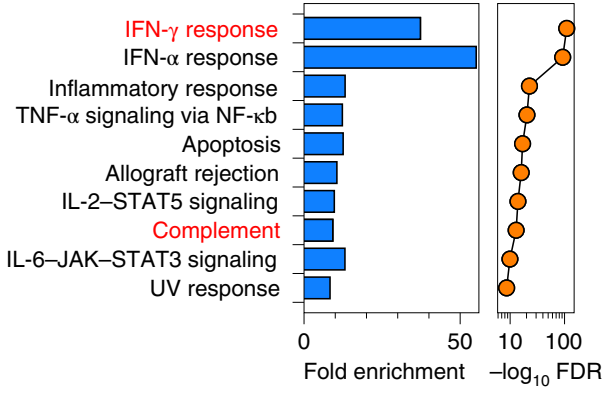

d

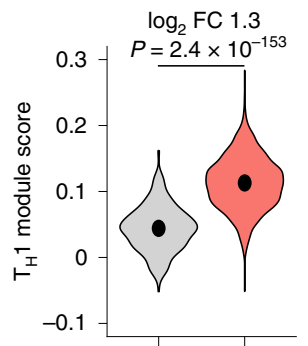

Healthy

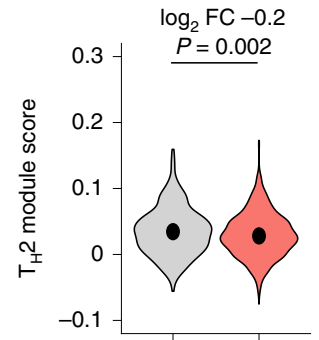

COVID-19

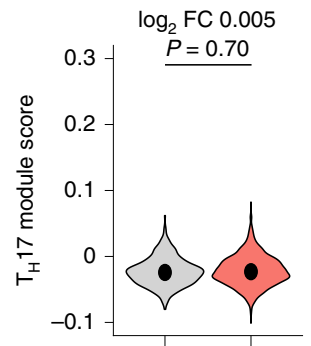

e Healthy COVID-19

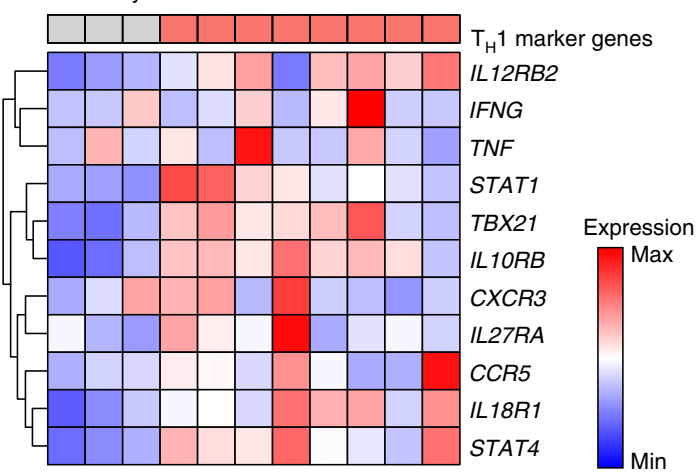

Fig. 1 | COVID-19 CD4+ helper $\mathbf{T}$ cells are $\mathbf{T}_{\mathbf{H}} 1$ skewed. $\mathbf{a}$, Uniform manifold approximation and projection (UMAP) of scRNA-seq showing sub-clustering of T cells from BALF of $n=8$ patients with COVID-19 and $n=3$ healthy controls. Stack bars (right) show cumulative cellularities across samples in patients and controls. Dot plot of marker genes for these clusters are shown in Extended Data Fig. 1c. NK, natural killer. b,c, Heat map showing DEGs (at least 1.5-fold change in either direction at Bonferroni adjusted $P<0.05$ using two-sided Wilcoxon rank-sum test) between helper T cells of $n=8$ patients with COVID-19 and $n=3$ healthy controls (b) and enrichment of those DEGs in Hallmark MSigDB gene sets (c). NF, nuclear factor; TNF, tumor necrosis factor. False discovery rate (FDR)-corrected $P$ values in c are from hypergeometric tests. Highlighted in red in $\mathbf{c}$ are Hallmark IFN- $\gamma$ response and complement pathways. d, Violin plots showing expressions of $T_{H} 1-, T_{H} 2-$ and $T_{H} 17$-specific genes, respectively, summarized as module scores, in BALF helper $T$ cells of patients with COVID-19 and healthy controls. Medians are indicated. Exact $P$ values have been calculated using two-tailed Wilcoxon tests. FC, fold change. e, Heat map showing mean expression of classic $T_{H} 1$ marker genes in BALF helper T cells of patients with COVID-19 and healthy controls. Data are sourced from GSE145926 and GSE122960.

lung injury that can be ameliorated by complement inhibition ${ }^{10}$. Emerging clinical trial evidence, from small numbers of treated patients, also points to potential benefits of complement targeting in COVID-19 (ref. ${ }^{11}$ ).

The complement system is both hepatocyte-derived and serum-effective, but also expressed and biologically active within cells. Notably, activated $\mathrm{CD}^{+} \mathrm{T}$ cells process $\mathrm{C} 3$ intracellularly to $\mathrm{C} 3 \mathrm{a}$ and C3b via cathepsin L (CTSL)-mediated cleavage ${ }^{12}$. We have recently shown that SARS-CoV2-infected respiratory epithelial cells express and process C3 intracellularly via a cell-intrinsic enzymatic system to $\mathrm{C} 3 \mathrm{a}$ and $\mathrm{C} 3 \mathrm{~b}^{13}$. This represents a source of local complement within SARS-CoV2-infected lungs, where plasma-derived complement is likely to be absent, and signifies the lung epithelial lining as a complement-rich microenvironment. Excessive complement and IFN- $\gamma$-associated responses are both known drivers of tissue injury and immunopathogenesis ${ }^{14,15}$. On $\mathrm{CD}^{+}{ }^{+} \mathrm{T}$ cells, C3b binds CD46, its canonical receptor, to sequentially drive $\mathrm{T}_{\mathrm{H}} 1$ differentiation followed by their shut down, represented by initial production of interferon (IFN) $-\gamma$ alone, then IFN- $\gamma$ together with interleukin (IL)-10, followed by IL-10 alone ${ }^{16}$. Expression of IL-10 by $\mathrm{T}_{\mathrm{H}} 1$ cells is a critical regulator of $\mathrm{T}_{\mathrm{H}} 1$-associated inflammation ${ }^{2}$. However, the exact molecular mechanisms governing orderly regulation of $\mathrm{T}_{\mathrm{H}} 1$ responses culminating in IL-10 expression remain poorly understood and may be critical in the recovery phase of COVID-19 and other $\mathrm{T}_{\mathrm{H}} 1$-mediated inflammatory diseases.

VitD is a fat-soluble pro-hormone carefully regulated by enzymatic activation and inactivation. Most VitD is synthesized in the skin on exposure to ultraviolet B (UVB) radiation from sunlight, then undergoes sequential hydroxylation to 25(OH)VitD and $1,25(\mathrm{OH})_{2} \mathrm{VitD}$, classically in the liver and kidneys, respectively. VitD has immunomodulatory functions, hence, VitD deficiency is associated with adverse outcomes in both infectious ${ }^{17}$ and autoimmune diseases ${ }^{18}$. There are compelling epidemiological associations between incidence and severity of COVID-19 and VitD deficiency/insufficiency ${ }^{19}$, but the molecular mechanisms remains unknown.

We found $\mathrm{T}_{\mathrm{H}} 1$-skewed $\mathrm{CD}^{+} \mathrm{T}$ cell responses in bronchoalveolar lavage fluid (BALF) of patients with COVID-19. As this is a complement-rich microenvironment, we investigated the molecular mechanisms governing orderly shutdown of $T_{H} 1$ responses induced by CD46 engagement. We found that CD46 induces a cell-intrinsic VitD signaling system, enabling $\mathrm{T}$ cells to both fully activate and respond to VitD. This process was primed by epigenetic remodeling and recruitment of four key transcription factors (TFs), VitD receptor (VDR), c-JUN, STAT3 and BACH2. Last, we examined these pathways in $\mathrm{CD}^{+} \mathrm{T}$ cells from the BALF of patients infected with SARS-CoV2 and found it to be impaired. 


\section{Results}

COVID-19 CD4 ${ }^{+}$cells show $\mathrm{T}_{\mathrm{H}} 1$ and complement signatures. We analyzed single-cell RNA-sequencing (scRNA-seq) data from the BALF and peripheral blood mononuclear cells (PBMCs) of patients with COVID-19 and healthy controls (GSE145926, GSE122960 and GSE150728). Because immunity to both SARS-CoV1 and MERS-CoV is mediated by, among other cells, IFN- $\gamma$-producing $\mathrm{CD}^{+}$memory $\mathrm{T}$ cells ${ }^{20}$ and development of $\mathrm{T}_{\mathrm{H}} 1$-polarized responses in SARS-CoV2 infection ${ }^{21}$ is suspected to contribute to pathogenic hyper-inflammation, we focused our analyses on $\mathrm{CD}^{+} \mathrm{T}$ cells. $\mathrm{T}$ cell populations within BALF (Extended Data $\mathrm{F}$ ig. 1a,b) comprised five major sub-clusters, including $\mathrm{CD}^{+}$ helper $\mathrm{T}$ cells, according to well-characterized markers (Fig. 1a and Extended Data Fig. 1c). Although the proportion of $\mathrm{T}$ cells that were $\mathrm{CD}^{+}$did not differ between patients and controls (Fig. 1a and Extended Data Fig. 1d), 312 genes were upregulated and 134 genes were downregulated in patients' $\mathrm{CD} 4^{+} \mathrm{T}$ cells (Fig. $1 \mathrm{~b}$ and Supplementary Table 1a). These differentially expressed genes (DEGs) were enriched in noteworthy biological pathways, including IFN- $\gamma$ response and complement (Fig. 1c and Supplementary Table 1b). Examination of transcriptional programs by module score indicated that $\mathrm{CD} 4^{+} \mathrm{T}$ cells in patients were preferentially polarized toward $\mathrm{T}_{\mathrm{H}} 1$, as opposed to type 2 helper $\mathrm{T}$ $\left(\mathrm{T}_{\mathrm{H}} 2\right)$ cells or the $\mathrm{T}_{\mathrm{H}} 17$ subset of helper $\mathrm{T}$ cell lineages (Fig. $1 \mathrm{~d}$ ). Consistently, expression of core $\mathrm{T}_{\mathrm{H}} 1$ marker genes were higher in patients (Fig. 1e).

Enrichment of complement pathway (Fig. 1c) was notable because (1) we recently identified complement as one of the most highly induced pathways in lung $\mathrm{CD}^{+}{ }^{+} \mathrm{T}_{\text {cells }}{ }^{22}$; (2) SARS-CoV2 potently induces complement, especially complement factor 3 (C3), from respiratory epithelial cells ${ }^{13}$; (3) COVID-19 lungs are a complement-rich microenvironment ${ }^{23}$; and (4) $\mathrm{CD}^{+} \mathrm{T}$ lymphocytes in COVID-19 lungs have a CD46-activated signature ${ }^{13}$. Because CD46 drives both $\mathrm{T}_{\mathrm{H}} 1$ differentiation and shutdown, characterized by IFN- $\gamma$ and IL-10 expression, respectively ${ }^{16}$, we determined the state of $\mathrm{T}_{\mathrm{H}} 1$ cells in COVID-19 BALF. IL10 mRNA was dropped out in scRNA-seq, but detectable in bulk RNA-seq from BALF (Extended Data Fig. 1e). Consistently we observed significant enrichment of $\mathrm{T}_{\mathrm{H}} 1$-related genes in patient cells compared to controls, but $\sim$ fivefold lower IL10 (Extended Data Fig. 1e). Similar examination within scRNA-seq of PBMCs (Extended Data Fig. 2a,b) did not show meaningful differences in $T_{H} 1, T_{H} 2$ or $T_{H} 17$ lineage genes (Extended Data Fig. 2c). Collectively, these data indicated the $\mathrm{T}_{\mathrm{H}} 1$ program and complement signature as features of helper $\mathrm{T}$ cells at the site of pulmonary inflammation where virus-specific $\mathrm{T}$ cells may be concentrated ${ }^{24}$ and are consistent the notion that COVID-19 $\mathrm{T}_{\mathrm{H}} 1$ cells were in the inflammatory phase of their lifecycle compared to healthy controls.
Complement induces an autocrine $\mathrm{T}$ cell VitD shutdown program. Prolonged and/or hyper- $\mathrm{T}_{\mathrm{H}} 1$ activity is pathogenic ${ }^{14,15}$. To discover how shutdown of $\mathrm{T}_{\mathrm{H}} 1$ cells could be accelerated, we explored how complement regulates $\mathrm{T}_{\mathrm{H}} 1$ shutdown in healthy cells. CD46, engaged by environmental or intracellularly generated $\mathrm{C} 3 \mathrm{~b}$, works co-operatively with $\mathrm{T}$ cell receptor signaling to drive $\mathrm{T}_{\mathrm{H}} 1$ differentiation then subsequent shutdown ${ }^{16}$. Thus, $\mathrm{T}$ cells activated with anti-CD3 and anti-CD46 produce IFN- $\gamma$, then co-produce IL-10 before shutting down IFN- $\gamma$ to produce only IL-10 (ref. ${ }^{16}$ ) (Fig. 2a). T cells secreting neither cytokine (IFN- $\gamma$ IL-10- cells) are also appropriately activated as they upregulate activation markers (CD25 and CD69; Extended Data Fig. 3a,b) and proliferate (Extended Data Fig. 3c,d). Because this CD46 system is not present in mouse $\mathrm{T}$ cells, we explored its function in human $\mathrm{CD} 4^{+} \mathrm{T}$ lymphocytes. Unless specified otherwise, we used regulatory $\mathrm{T}\left(\mathrm{T}_{\text {reg }}\right)$ cell helper $\mathrm{T}$ cell-depleted $\mathrm{CD} 4^{+}$helper $\mathrm{T}$ cells $\left(\mathrm{CD} 4{ }^{+} \mathrm{CD} 25^{-}\right)$throughout. After anti-CD3+anti-CD46 activation, we flow-sorted cells from each quadrant by surface cytokine capture (Fig. 2a) and performed transcriptome analysis (Extended Data Fig. 4a-c). Comparing transcriptomes of IFN- $\gamma^{+} \mathrm{IL}-10^{-}$, IFN $-\gamma^{+}$IL- $10^{+}$and IFN- $\gamma^{-}$IL- $10^{+}$against IFN- $\gamma^{-}$IL- $10^{-}$helper T cells, $\sim 2,000$ DEGs were in common (Fig. 2b, Extended Data Fig. 4d and Supplementary Table 1c,d). These were enriched for proteins whose molecular function pertained to TF biology (Extended Data Figs. 2c and $4 \mathrm{e}, \mathrm{f}$ and Supplementary Table 1e), indicating that a key role of CD46 is to regulate TFs. In total, 24 TFs were induced by CD46 in cytokine producing $\mathrm{CD}^{+}$cells (Fig. 2d), including VDR (Fig. 2d). VDR was notable for two reasons. First, independent prediction of TFs regulating DEGs of BALF CD4 ${ }^{+} \mathrm{T}$ cells and lung biopsies of COVID-19 versus healthy donors returned VDR among the top candidates (Fig. 2e and Supplementary Table 1f). Second, CYP27B1 was concurrently induced in the transcriptome data (Fig. 2d). CYP27B1 is the $1 \alpha$-hydroxylase catalyzing the final activation of VitD, converting $25(\mathrm{OH}) \mathrm{VitD}$ to biologically active $1,25(\mathrm{OH})_{2}$ VitD. Inducible expression of CYP27B1 and VDR in helper T cells indicated the likely presence of an autocrine/paracrine loop, whereby $\mathrm{T}$ cells can both activate and respond to VitD. Although both are described in activated $\mathrm{T}$ cells ${ }^{25,26}$, the molecular mechanism and consequences are unknown. Anti-CD3 + anti-CD28 stimulation of $\mathrm{T}$ cells activates $\mathrm{C} 3$ processing intracellularly by CTSL, generating autocrine $\mathrm{C} 3 \mathrm{~b}$ to ligate $\mathrm{CD} 46$ on the cell surface ${ }^{12}$, which in turn enhances $\mathrm{C} 3$ processing to further generate $\mathrm{C} 3 \mathrm{~b}^{12}$. To establish that $C Y P 27 B 1$ and VDR are induced by complement, we confirmed that anti-CD3 + anti-CD28 and anti-CD3 + anti-CD46 both stimulate these genes in T cells and that this effect was nullified by a cell-permeable CTSL inhibitor, which blocks intracellularly generated C3b (Fig. 2f). Similarly, T cells from CD46-deficient patients did not upregulate CYP27B1 or VDR with either stimulus (Fig. 2g).

Fig. 2 | VDR and CYP27B1 are induced by complement and predicted as regulators of the $T_{H} 1$ program in COVID-19. a, Representative flow cytometry showing IFN- $\gamma$ and IL-10 in CD4 ${ }^{+}$helper T cells activated with anti-CD3 + anti-CD46 and the four quadrants (A, B, C and D) from which cells were flow-sorted for transcriptome analysis. Live and single cells are pre-gated. $\mathbf{b}$, Venn diagram showing number of $D E G s( \pm \geq 1.5$-fold at unadjusted $P<0.05$ using analysis of variance (ANOVA)) comparing cells in quadrants B, C and D against A, respectively ( $n=4$ experiments). c, Enrichment of Gene Ontology molecular function terms in shared DEGs (intersect of Venn diagram in b), ranked by statistical significance. Marked are terms corresponding to TF activity. $\mathbf{d}$, Heat map of induced TFs in anti-CD3 + anti-CD46-activated helper T cells at each stage of the lifecycle shown in $\mathbf{a}$. Highlighted are VDR and expression of CYP27B1. e, EnrichR-predicted ENCODE and ChIP enrichment analysis TFs regulating the DEGs between COVID-19 versus healthy donor helper T cells (top) and lung biopsies (bottom). Shown are Benjamini-Hochberg adjusted $P$ values from hypergeometric tests. f, VDR (left) and CYP27B1 (right) $\mathrm{mRNA}$, in helper T cells activated or not, as indicated, with or without cathepsin L inhibitor (CTSL inh.) ( $n=5$ experiments). $\mathbf{g}$, VDR (left) and CYP27B1 (right) mRNA in helper T cells of a patient with CD46-deficiency, activated or not, as indicated ( $n=3$ experiments). h,i, Representative flow cytometry $(\mathbf{h})$ and cumulative data from $n=6$ independent experiments (i) showing IFN- $\gamma$ and IL-10 in helper T cells activated with anti-CD3 + anti-CD46 with or without carrier, active $(1,25(\mathrm{OH}) 2 \mathrm{D} 3)$ or inactive $(25(\mathrm{OH}) \mathrm{D} 3) \mathrm{VitD}$. j, IL10 in helper T cells activated with anti-CD3 + anti-CD46 with or without inactive (25(OH)D3) VitD, with siRNA targeting VDR, CTSL or CYP27B1 or non-targeting (NT) siRNA ( $n=5$ experiments). Data in a-d are from GSE119416. Data in e (top) are from GSE145926 and GSE122960. Data in e (bottom) are from GSE147507. Data in $\mathbf{g}$ are from microarrays ${ }^{54}$. Bars in $\mathbf{f}, \mathbf{g}$,i show mean + s.e.m. Box plots in $\mathbf{j}$ show median value and the range extends from minimum to maximum. All statistical tests are two-sided. ${ }^{\star} P<0.05{ }^{\star \star} P<0.01$ ${ }^{\star \star \star} P<0.001^{\star \star \star \star} P<0.0001$ by ANOVA. 
Collectively, these data indicated that CD46 ligation by complement induces both the enzyme and receptor to enable helper $\mathrm{T}$ cells to fully activate and respond to VitD.

As proof of principle that this autocrine/paracrine intracellular VitD system is involved in $\mathrm{T}_{\mathrm{H}} 1$ shutdown, we stimulated $\mathrm{CD}^{+}{ }^{+} \mathrm{T}$ cells with anti-CD3 + anti-CD46 with or without active $\left(1,25(\mathrm{OH})_{2}\right)$ or inactive $(25(\mathrm{OH}))$ VitD. Both forms of VitD significantly repressed IFN- $\gamma$ and increased IL-10 (Fig. 2h,i), indicating that the cells had acquired the ability to activate and respond to VitD. We cultured similarly stimulated cells with inactive VitD and silenced CTSL, VDR or CYP27B1 using siRNA and assessed IL10 transcription. A significant reduction in IL10 was evident on silencing any of these components (Fig. $2 \mathrm{j}$ and Extended Data Fig. 5), indicating that they are all required for producing IL-10. Altogether, these data indicated existence of a complement-induced intracellular autocrine/paracrine VitD system promoting T cell shutdown.

VitD induces IL-10 via autocrine/paracrine IL-6/STAT3. We next investigated the effects of $\mathrm{VitD}$ on $\mathrm{CD}^{+} \mathrm{T}$ cells. In all subsequent experiments cells were activated with anti-CD3 and anti-CD28 (as this stimulus also signals through CD46 (ref. ${ }^{12}$ )) and we used active $1,25(\mathrm{OH})_{2}$ VitD. We confirmed that VDR protein was induced by T cell activation (Extended Data Fig. 6a), VitD-enhanced VDR expression ${ }^{27}$ (Extended Data Fig. 6a) and VitD-bound VDR

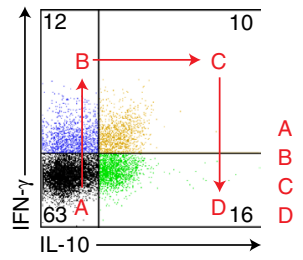

Cell sorting and transcriptome

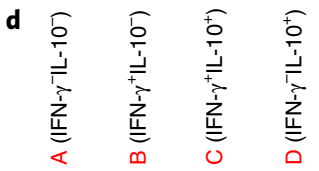
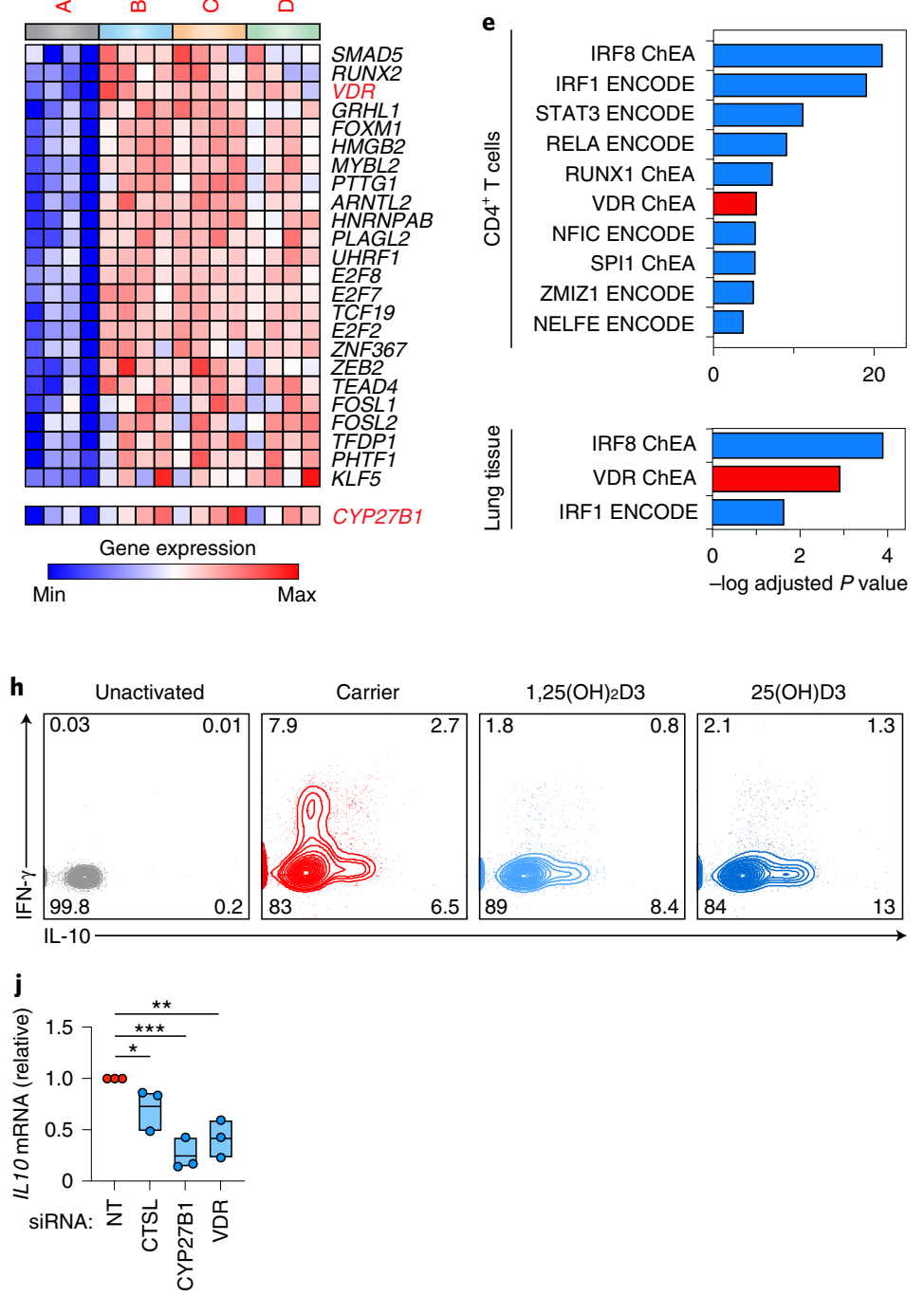

- $\log$ adjusted $P$ value b

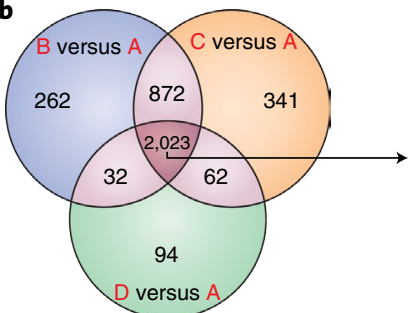

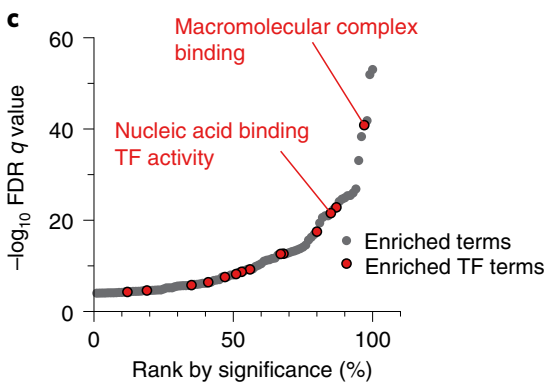
$\square$ Unactivated
Anti-CD3 + anti-CD28
Anti-CD3 + anti-CD46
Anti-CD3 + anti-CD28 + CTSL inh.
Anti-CD3 + anti-CD46 + CTSL inh.
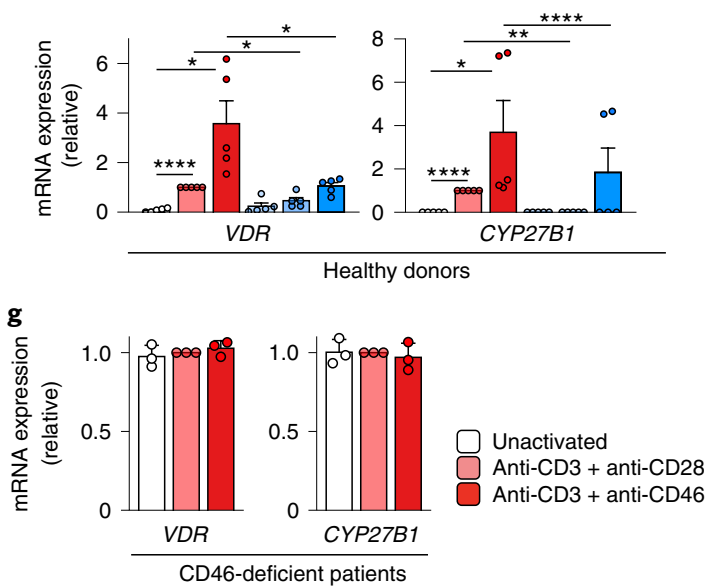

i

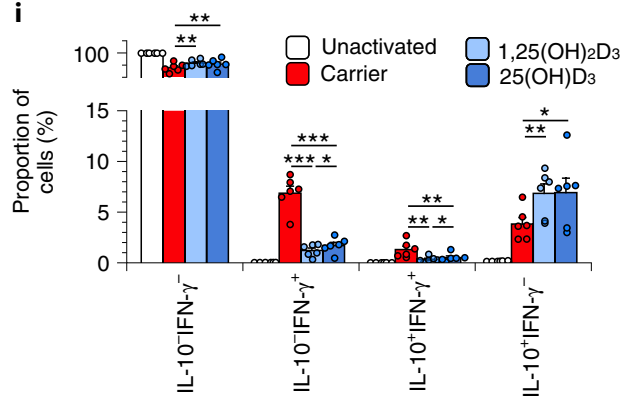


translocated to the nucleus (Extended Data Fig. 6b,c). VitD upregulated 262 genes and downregulated 128 genes in helper T cells (Fig. $3 a$ and Supplementary Table 2a,b), which was not due to alterations in cell proliferation or death (Extended Data Fig. 6d). Classical VitD-regulated genes, including CTLA4, CD38 and CYP24A1, were induced and both type 1 (IFNG) and type 17 (IL17A, IL17F, IL22 and IL26) cytokines were repressed (Fig. 3b,c), consistent with previous reports ${ }^{28}$. Genes induced by VitD included two cytokines, IL10 and IL6 and several TFs, including JUN, BACH2 and STAT3 (Fig. 3b,c). As IL-10-producing CD4 ${ }^{+}$FoxP3- $\mathrm{T}_{\text {reg }}$ type 1 ( $\left.\operatorname{Tr} 1\right)$ cells can be induced from naive helper $\mathrm{T}$ cells by $\mathrm{VitD}^{29}$, we noted that genes induced/repressed by VitD were not enriched in $\operatorname{Tr} 1$ cell signature genes (Extended Data Fig. 6e) and did not exhibit the archetypal

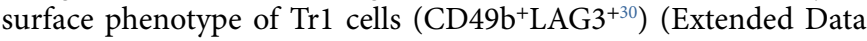
Fig. 6f). Similarly, FOXP3, the master TF of natural $\mathrm{T}_{\text {reg }}$ cells, was not upregulated by VitD (Supplementary Table $2 \mathrm{~b}$ ). Genes regulated by VitD were most significantly enriched for cytokines (Extended Data Fig. 6g). Unexpectedly, IL6, usually a pro-inflammatory cytokine, was the most highly induced gene in the hierarchy of cytokines regulated by VitD (Extended Data Fig. 7a). We confirmed repression of IFN- $\gamma$ and IL-17 and induction of IL-10 and IL- 6 proteins in VitD-treated helper T cells (Extended Data Fig. 7b) and noted a strong dose-response relationship between VitD concentrations and these effects (Fig. 3d). To confirm again the autocrine/paracrine VitD activation system at the protein level (Extended Data Fig. 5c), we observed repression of IFN- $\gamma$ and IL-17 and induction of IL-10 and IL- 6 by helper T cells treated with only inactive $25(\mathrm{OH})$ VitD, indicating intracellular conversion of $25(\mathrm{OH}) \mathrm{VitD}$ to active 1,25(OH $)_{2}$ VitD (Extended Data Fig. 7d).

IL-6 is a pleiotropic cytokine expressed by most stromal and immune cells ${ }^{31}$. Yet it is not commonly attributed to $\mathrm{CD}^{+} \mathrm{T}$ cells. We established that IL6 mRNA and protein were produced by $\mathrm{T}$ cells and induced by VitD (Extended Data Fig. 7e) and that VitD treatment significantly increased intracellular expression of IL-6 (Extended Data Fig. 7f). In these experiments, there was a strong correlation between IL- 6 and IL-10 produced in response to VitD (Fig. 3e), suggesting a potential causal relationship. Accordingly, we stimulated helper T cells with VitD, with or without tocilizumab, an IL-6 receptor (IL-6R)-blocking antibody used clinically to treat IL-6-dependent cytokine release syndrome, including that seen in COVID-19 (ref. ${ }^{32}$ ). Tocilizumab significantly impaired IL-10 produced by VitD, indicating that IL-6R signaling induced by autocrine/paracrine IL- 6 promotes IL-10 in VitD-treated helper T cells (Fig. 3f). IL-6 can cooperate with IL-27 (ref. ${ }^{33}$ ) or IL-21 (ref. ${ }^{34}$ ) to promote IL-10 production in mouse T cells. However, both these cytokines were repressed by VitD in our transcriptomic analyses (Supplementary Table 2b), so it did not seem likely that these cytokines cooperate with IL-6 to induce IL-10 in this setting.
Addition of exogenous IL-6 without VitD did not induce IL-10 (Extended Data Fig. 7g) but increased pro-inflammatory IL-17 (Extended Data Fig. 7h), as has been reported ${ }^{35}$. These data indicate that pro-inflammatory functions of IL-6 may be restricted or averted by the production of anti-inflammatory IL-10 in the presence of VitD in human helper T cells (Fig. 3f).

Genes differentially expressed by VitD were enriched in cytokine signaling pathways, which are commonly mediated through phosphorylation of signal-dependent TFs, including JAK-STATs (Extended Data Fig. 6g). We therefore carried out a phospho-kinase protein array using lysates of carrier and VitD-treated cells. Five proteins showed significant differences in phosphorylation between carrier and VitD, most notably c-JUN and STAT3, both of which were significantly more phosphorylated (Fig. 3g,h and Extended Data Fig. 7i). Immunoblotting confirmed induction of both STAT3 protein and STAT3 phosphorylation by VitD (Fig. 3i and Extended Data Fig. 7j). IL-6 potently drives STAT3 activation by phosphorylation $^{31}$. STAT3 phosphorylation induced by VitD was abrogated by blockade of the IL-6R with tocilizumab, indicating that VitD-induced IL-6 is responsible for STAT3 phosphorylation (Fig. $3 \mathrm{i}$ and Extended Data Fig. 7j). Conversely, STAT3 protein expression was dependent on VitD but independent of IL-6 signaling, as IL-6R blockade did not reverse its induction by VitD (Fig. 3i and Extended Data Fig. 7j). By contrast, both c-JUN expression and phosphorylation were dependent on VitD and mostly independent of IL-6 (Fig. $3 \mathrm{i}$ and Extended Data Fig. 7j). As VitD-induced STAT3 phosphorylation was mediated by IL-6, we investigated whether STAT3 drives IL-10 produced by VitD. Both a cell-permeable STAT3 inhibitor and knockdown of STAT3 by siRNA significantly impaired IL-10 produced in response to VitD (Fig. 3j,k and Extended Data Fig. 7k,l). Likewise, VitD failed to produce significant IL-10 from helper T cells of patients with hyper-IgE syndrome, which have dominant negative STAT3 mutations and are unable to transduce STAT3 signaling normally (Fig. 3j). Collectively, these data established that VitD induces STAT3 and IL-6 and autocrine/paracrine IL-6R engagement phosphorylates STAT3, which promotes production of IL-10.

\section{VitD alters epigenetics and recruits c-JUN, STAT3 and BACH2.} VitD-bound VDR interacts with histone acetyl transferases, transcriptional co-activators, co-repressors and chromatin remodeling complexes to modulate transcription. We explored the effects of VitD on $\mathrm{T}$ cell epigenetic landscapes, using memory $\mathrm{CD}^{+} \mathrm{T}$ cells (these cells express VDR without requiring pre-activation). We profiled genome-wide histone 3 lysine 27 acetylation (H3K27Ac), a marker of active regions of the genome, using cleavage under target and release using nuclease (CUT\&RUN) in VitD and carrier-treated cells. VitD induced dynamic changes in histone acetylation genome-wide (Fig. 4a,b), indicating significant changes

Fig. 3 | VitD induces IL-10 in helper T cells by enhancing IL-6-STAT3 signal transduction. a, Number of DEGs between VitD and carrier-treated helper T cells $( \pm \geq 1$.5-fold change at FDR $<0.05)$. b. Scatter-plot showing mRNA expression (RPKM) of genes in helper T cells treated with VitD or carrier. VitD-induced and -repressed genes are depicted in red and blue, respectively. Noteworthy genes are annotated (black), including classical VitD-induced genes (orange); $n=3$ independent biological experiments. c, Heat map showing expression of select genes from $\mathbf{b}$. d, Dose-response of indicated cytokines from helper T cells treated for $72 \mathrm{~h}$ with VitD. Stars indicate statistically significant changes in comparison to $0 \mathrm{nM}$ of $\mathrm{VitD} ; n=3$ experiments. e, Pearson correlation between IL- 6 and IL-10 concentrations in culture supernatants of VitD-treated helper T cells. Shown is the correlation line, plus 95\% confidence interval. f, IL-10 concentrations in supernatants of helper T cells cultured with VitD, with and without tocilizumab; $n=3$ experiments. g, Representative image from $n=2$ experiments of a phospho-kinase array (array of 43 kinases in duplicate spots) carried out on 3-d lysates of carrier- or VitD-treated s. Location of STAT3 phosphorylated at lysine 705, c-JUN phosphorylated at serine 63 and reference spot (to which all spots are normalized) are indicated. $\mathbf{h}$, Heat map showing normalized phosphorylation values of differentially phosphorylated proteins following VitD treatment (Extended Data Fig. 7i) in $n=2$ donors. $\mathbf{i}$, Immunoblots of lysates of helper T cells treated with carrier or VitD with and without, tocilizumab (Toc) at the concentrations shown. Shown are representative images from $n=3$ experiments (quantified in Extended Data Fig. 7j). j, IL-10 production from helper T cells cultured with carrier or VitD, with or without a STAT3 inhibitor (STAT3i). Genotype of cells (WT, STAT3 wild type; DN, STAT3 dominant negative) is indicated; $n=3$ experiments. Each dot represents an individual donor. k, IL-10 production from helper T cells transfected with control siRNA or siRNA targeting STAT3; $n=5$ experiments. Unless indicated, all cells were activated with anti-CD3 + anti-CD28. Cumulative data in d,f,j,k depict mean + s.e.m. All statistical tests are two-sided. ${ }^{\star} P<0.05,{ }^{\star \star} P<0.01,{ }^{\star \star \star} P<0.001,{ }^{\star \star \star \star} P<0.0001$ by one-way $(\mathbf{d}, \mathbf{j}, \mathbf{k})$ and two-way ANOVA (f). 
in enhancer architecture. By $48 \mathrm{~h}$ after VitD treatment, $\sim 25,000$ and $\sim 21,000 \mathrm{H} 3 \mathrm{~K} 27 \mathrm{Ac}$ peaks were induced and repressed, respectively (Fig. 4c,d and Supplementary Table 3a). Loci of genes transcriptionally induced by VitD showed increased histone acetylation and those repressed by VitD had reduced histone acetylation (Fig. 4c). The size of these peaks was significantly affected by VitD, leading to generation of new super-enhancer (SE) architectures and promotion of existing SEs (Fig. 4e). SEs are complex regulatory domains consisting of collections of enhancers critical for regulating genes of particular importance to cell identity and risk of genetic disease $^{36,37}$. VitD-modified SEs included those associated with $B A C H 2, S T A T 3, I L 10$ and other genes induced by VitD (Fig. 4e and Supplementary Table $3 \mathrm{~b}$ ). To identify potential TFs recruited to these loci, we carried out TF DNA motif finding at H3K27Ac peak loci induced by VitD. The top enriched motifs were VDR, AP-1 family members, notably c-JUN and BACH2, and STAT3 (Fig. 4f). All three of these were TFs transcriptionally induced by VitD (Fig. 3b,c) and two of these, c-JUN and STAT3, were also a
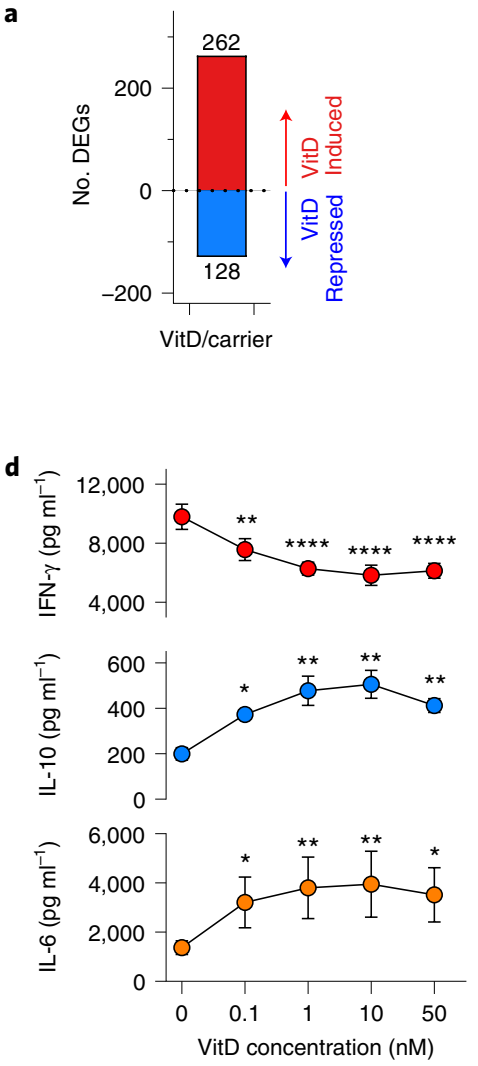

g

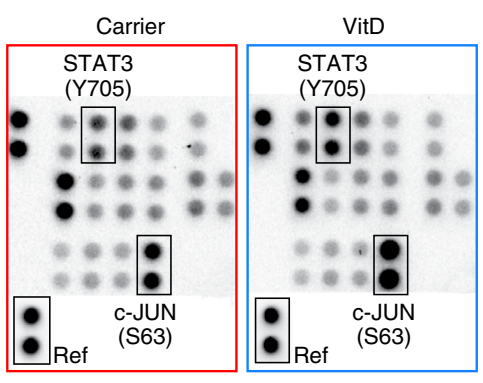

h
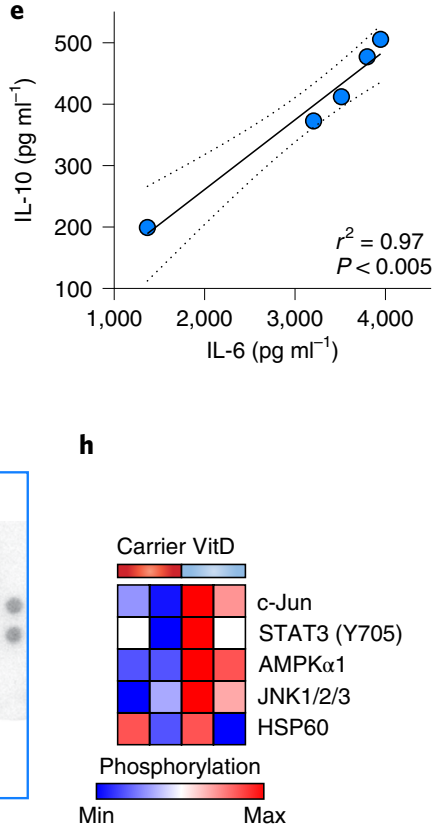
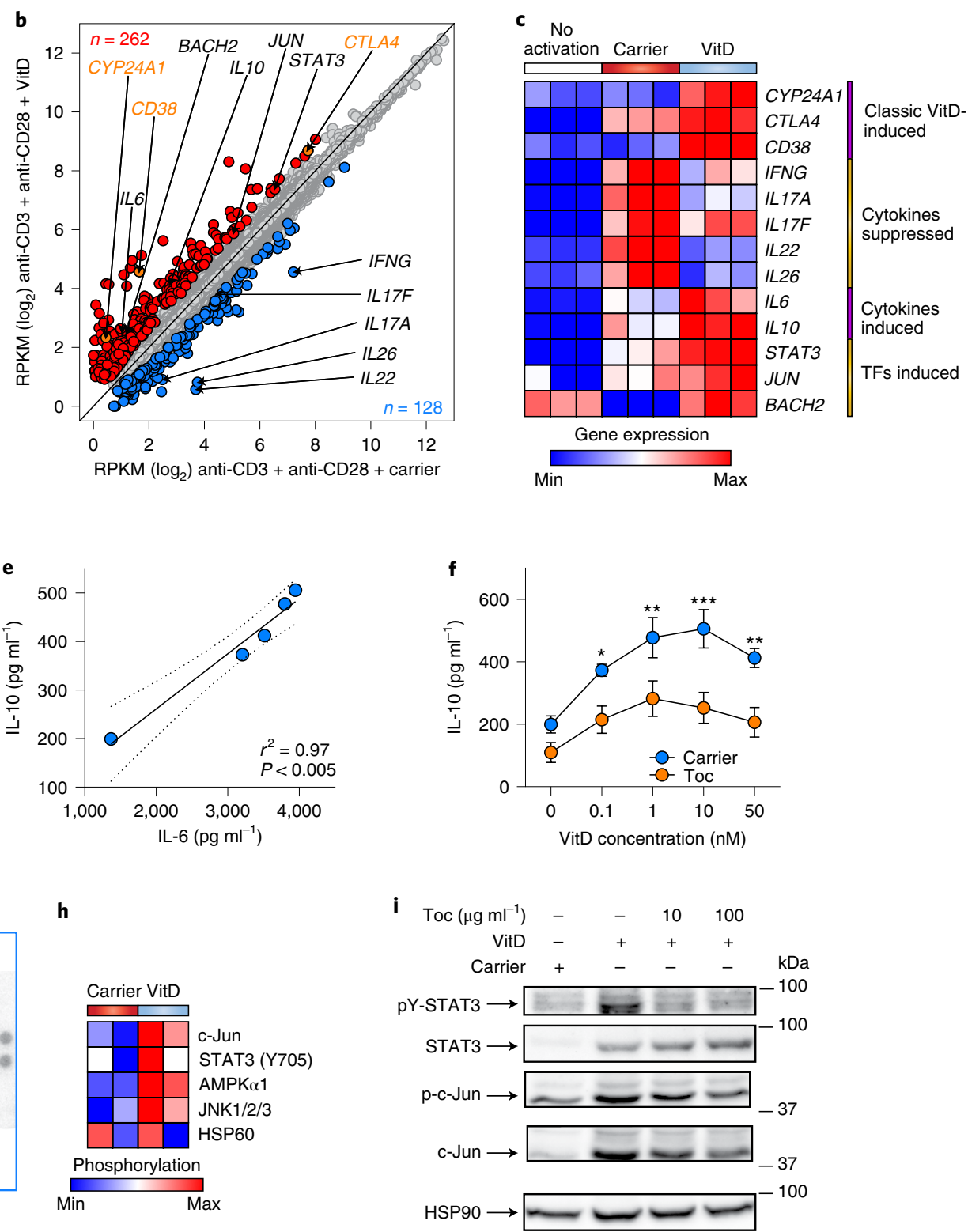

j

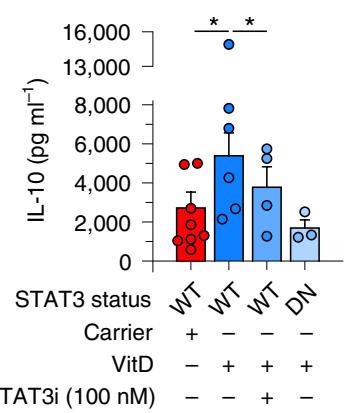

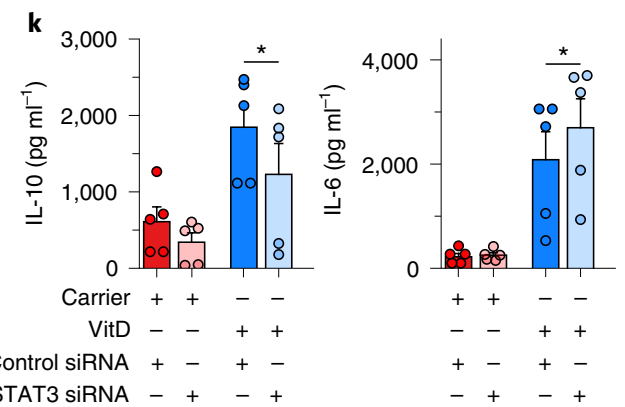


a
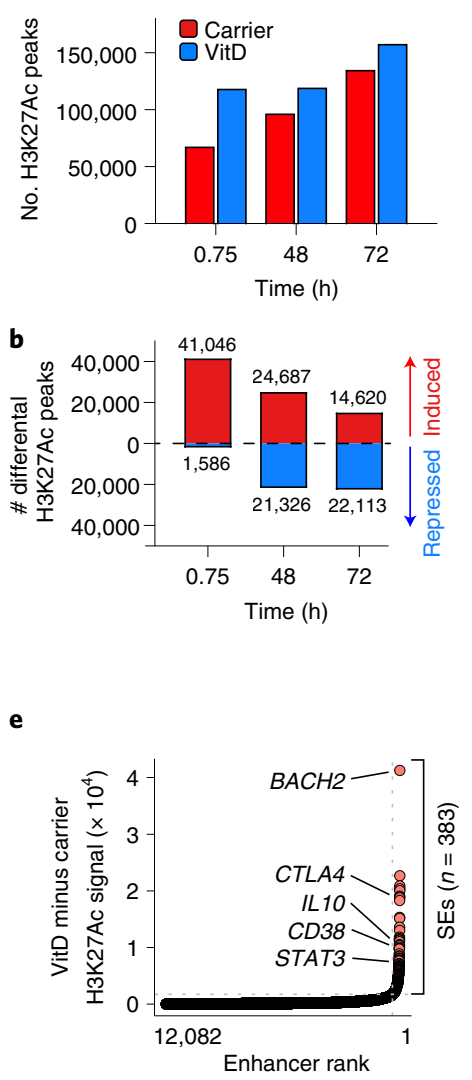

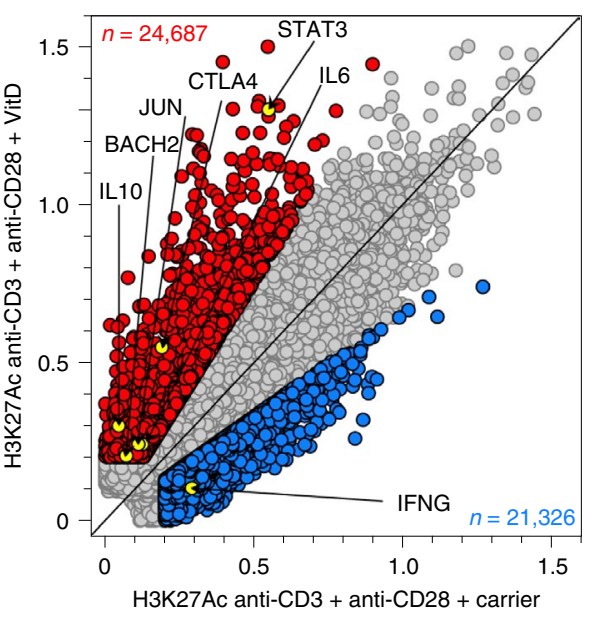

$\mathbf{f}$ d

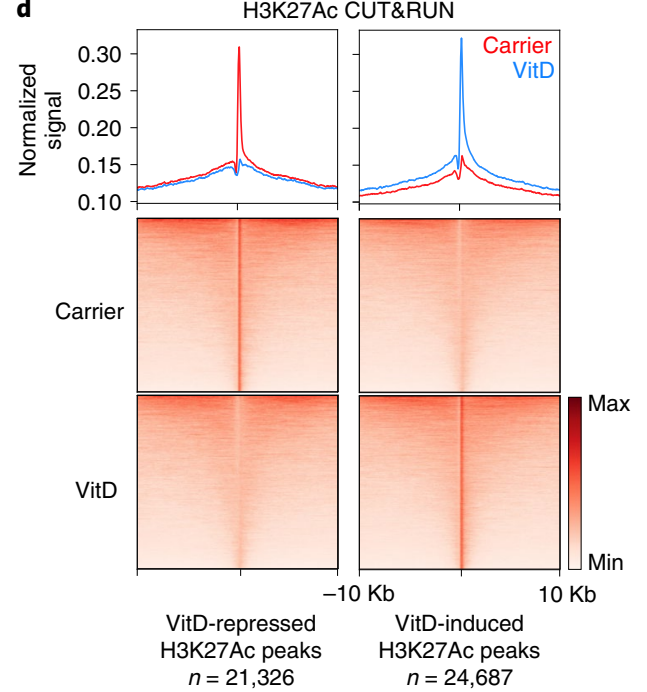

Fig. 4 | VitD reshapes epigenetic landscape of helper T cells. a, Genome-wide H3K27Ac CUT\&RUN peaks 45 min, $48 \mathrm{~h}$ and $72 \mathrm{~h}$ after VitD or carrier-treatment of helper T cells. b. Differential H3K27Ac peaks (signal $\geq 0.2, \geq 1.5 \times \mathrm{FC}$ ) after VitD or carrier-treatment of helper T cells at the indicated time points. c, Scatter-plot showing H3K27Ac CUT\&RUN peak signal intensities $48 \mathrm{~h}$ after VitD or carrier-treatment of helper T cells. Indicated are VitD-induced peaks (red) and VitD-repressed peaks (blue). Highlighted are select peaks at loci of interest. Data show a representative example from $n=2$ independent experiments. d, Heat maps showing H3K27Ac signal at VitD-repressed and VitD-induced peaks (bottom) and histograms showing normalized signals in carrier and VitD-treated cells (48h) (top). e, Ranked order of H3K27Ac-loaded enhancers induced by VitD in helper T cells after $48 \mathrm{~h}$. SEs are indicated. Marked are the relative positions, ranked according to signal intensity (higher indicates greater signal intensity), of enhancers attributed to selected genes. f, Enriched TF DNA motifs at H3K27Ac peak loci induced by VitD. Shown are TF families on the left and representative TF members enriched in the data on the right. Unless indicated, all in vitro T cell experiments depicted in Fig. 4 have been activated with anti-CD3 + anti-CD28.

post-transcriptionally more phosphorylated after VitD (Fig. 3g,h). Thus, we reasoned that they are likely to play an important role in gene regulation by VitD.

We carried out c-JUN CUT\&RUN and VDR, STAT3 and BACH2 cleavage under target and tagmentation (CUT\&Tag) in VitD- or carrier-treated cells (Extended Data Fig. 8a and Supplementary Table 4) to identify genome-wide distribution of these TFs. Genomewide binding of all four TFs was increased after VitD (Fig. 5a) and they were recruited to H3K27Ac peak loci induced by VitD (Fig. 5b and Extended Data Fig. 8b). VDR, c-JUN, STAT3 and BACH2 each bound $\sim 20-40 \%$ of genes differentially expressed by VitD, significantly higher than other loci in the genome (Fig. $5 \mathrm{c}$ ). Indeed, $\sim 60 \%$ of DEGs were bound by at least one of these TFs (Fig. 5d), half of which were bound by more than one (Fig. $5 \mathrm{e}$ ). Genes bound by two or more TF included STAT3, IL10 and BACH2 (Fig. 5f), indicating the complexity of gene regulation downstream of VitD exposure and interaction of multiple TFs within a gene regulatory network (Fig. 5g). Collectively, these data showed reshaping of the epigenome by VitD, generating new and augmenting existing enhancers and recruitment of transcriptional regulators to these loci to modify transcriptional output. BACH2, IL10 and STAT3 were exemplars of loci at which VDR binding generated new SEs, to which c-JUN, BACH2 and STAT3 were recruited (Fig. 5h and Extended Data Fig. 8c,d) and transcriptional output was increased (Fig. 3b,c).

We noted that STAT3 is directly bound by VDR (Fig. $5 f$,g and Extended Data Fig. 8d), but we did not find a CUT\&Tag VDR peak proximal to the IL6 locus. This may be because IL6 is a lower affinity target, has a distal VDR binding site or because VDR binds this site earlier than the time point at which we carried out CUT\&Tag. Thus, because ChIP-qPCR is more sensitive than genome-wide techniques when applied to individual loci, we performed qPCR for STAT3 and IL6 promoters, as well as CYP24A1 (a positive control locus), in anti-VDR ChIP fragments (Fig. 5i). We found strong enrichment of CYP24A1 and STAT3 promoters and moderate enrichment of the IL6 promoter in anti-VDR ChIP fragments, indicating that these loci are all directly bound by VDR and that 
VDR binding at IL6 is at lower affinity than the other two loci (Fig. 5i). In summary, these data indicated VitD-induced dynamic changes in genome-wide enhancer architecture and recruitment of several TFs to loci that could explain $\sim 60 \%$ of the VitD-dependent variance in gene expression.

BACH2 regulates the VitD response in $\mathrm{CD}^{+} \mathrm{T}$ cells. $\mathrm{BACH} 2$ is a critical immunoregulatory $\mathrm{TF}^{38,39}$. We confirmed that VitD induces $\mathrm{BACH} 2$ protein expression in helper $\mathrm{T}$ cells (Fig. 6a). Psoriatic skin is rich in $\mathrm{CD}^{+} \mathrm{T}$ cells, psoriasis severity is associated with low active VitD levels ${ }^{40}$ and is frequently treated successfully with $V_{\text {VitD }}{ }^{41}$. We therefore performed confocal imaging on the dermis of patients with psoriasis treated, or not, with VitD. VitD treatment significantly increased numbers of $\mathrm{BACH} 2^{+}$cells and greater numbers of intranuclear foci of $\mathrm{BACH} 2$, compared to untreated skin (Fig. 6b-e), confirming that $\mathrm{BACH} 2$ is induced by VitD in vivo. Genes regulated by VitD were $\sim$ two- to threefold enriched for $\mathrm{BACH} 2$ binding than those not regulated by VitD (Fig. 6f,g). Indeed, $\mathrm{BACH} 2$-induced genes and $\mathrm{BACH} 2$-repressed genes were more highly expressed in VitD-treated and carrier-treated cells, respectively (Extended Data Fig. 9a,b), indicating a $\mathrm{BACH} 2$ signature in VitD-regulated genes. Among the most highly enriched in the leading edge of BACH2-induced genes was the IL-6 receptor $\alpha$-chain (IL6R) (Extended Data Fig. 9a,c). These observations suggested that a significant proportion of VitD-driven transcription is BACH2-dependent. Thus, we compared transcriptomes of VitD-treated helper $\mathrm{T}$ cells from a healthy control $\left(B A C H 2^{\mathrm{WT} / \mathrm{WT}}\right)$ to those from a $B A C H 2$ haploinsufficient patient $\left(B A C H 2^{\mathrm{WT} / \mathrm{L} 24 \mathrm{P}}\right)^{36}$. VitD-induced genes were significantly enriched in the transcriptomes of wild-type cells (Fig. $6 \mathrm{~h}$ and Supplementary Table 5a), indicating that normal $\mathrm{BACH} 2$ concentrations are required for appropriate regulation of VitD-induced genes (Extended Data Fig. 9e). The same pattern of enrichment was not found for VitD-repressed genes (Extended Data Fig. 9d), potentially indicating that half-normal concentrations of $\mathrm{BACH} 2$ are sufficient for repression of VitD targets. Thus, some, but not all, type 1 and type 17 inflammatory cytokines (IFNG and IL17F, but not IL17A) were repressed by VitD in BACH2 haploinsufficient cells (Extended Data Fig. 9e and Supplementary Table 5b).

To better understand the VitD transcriptional regulatory network, we integrated VitD up- and downregulated genes in $B A C H 2^{\mathrm{WT} / \mathrm{WT}}$ and $B A C H 2^{\mathrm{WT} / \mathrm{L} 24 \mathrm{P}} \mathrm{CD} 4^{+} \mathrm{T}$ cells together with TF binding from CUT\&Tag and CUT\&RUN (Fig. 6i). The 75\% and $66 \%$ of genes normally up- and downregulated by VitD, respectively, were not regulated by VitD in $\mathrm{BACH} 2$ haploinsufficient cells. Of these, $28 \%$ and $14 \%$, respectively, were directly bound by $\mathrm{BACH} 2$ (Fig. 6j) and functionally annotated as cytokine and cytokinecytokine receptor signaling genes, including IL6R and IL10 (Fig. 6k). As noted, VitD promoted IL-10 via IL-6 signaling through IL-6R and STAT3 (Fig. 3f-j). Despite higher basal messenger RNA, IL6 was still induced by VitD when BACH2 levels were sub-normal, but IL10 was not, signifying that the IL-6-STAT3-IL-10 axis was disrupted in BACH2 haploinsufficiency (Fig. 6i-1 and Extended Data Fig. 9e). Indeed, IL6R was not appropriately induced when $\mathrm{BACH} 2$ levels were sub-normal (Fig. 6i-1 and Extended Data Fig. 9e). In animals, the IL6r gene is a direct genomic target of Bach2 and Bach2 knockout status significantly impairs expression of Il6r (Extended Data Fig. 9f). Similar binding of this gene by BACH2 was evident in human cells (Fig. $6 \mathrm{~m}$ ), presumably explaining why $\mathrm{BACH} 2$ haploinsufficiency impaired IL6R expression. As BACH2 also binds IL10 (Fig. 5h), it is likely that BACH2 deficiency also contributes directly to lower VitD-induced IL10 transcription. Collectively, these data indicate that $\mathrm{BACH} 2$ is a VitD-induced protein regulating a significant portion of the VitD transcriptome, with a key role in mechanisms that stimulate expression of IL-10.

VitD is predicted to retract the $T_{H} 1$ program in COVID-19. There is compelling epidemiological association between incidence and severity of COVID-19 and VitD deficiency/insufficiency ${ }^{19}$, but the molecular mechanisms remain unknown. Given preferential $\mathrm{T}_{\mathrm{H}} 1$ polarization in COVID-19 BALF (Fig. 1d and Extended Data Fig. 1e), we hypothesized that VitD could be mechanistically important for hyper-inflammation in COVID-19 and may be a therapeutic option. We studied expression of VitD-regulated genes (Fig. 3a and Supplementary Table 2b) in COVID-19 BALF helper T cells. Expression of VitD-repressed genes, summarized as module score, was significantly higher in patient helper $\mathrm{T}$ cells than healthy controls (Fig. 7a). This was further corroborated by gene set enrichment analysis (GSEA) showing that genes more highly expressed in patient compared to control cells were enriched in VitD-repressed genes (Fig. 7b). On a per-cell basis the VitD-repressed module score correlated strongly with the $\mathrm{T}_{\mathrm{H}} 1$ score in BALF helper $\mathrm{T}$ cells (Fig. 7c), indicating a reciprocal relationship between $\mathrm{T}_{\mathrm{H}} 1$ genes and VitD-repressed genes. By contrast, VitD-induced genes were not substantially different (Extended Data Fig. 10a), nor were VitD-regulated genes different in PBMCs of patients compared to healthy donors (Extended Data Fig. 10b). To assess the ability of the $\mathrm{T}_{\mathrm{H}} 1$ and VitD-repressed gene signatures to distinguish patient from healthy BALF helper T cells on a per-cell basis, we constructed receiver operating characteristic (ROC) curves of the module scores. The area under the curve (AUC) for the two scores were 0.91 and 0.80 , respectively $(P<0.00001$ for both) (Fig. $7 \mathrm{~d})$. Thus, both $\mathrm{T}_{\mathrm{H}} 1$ and VitD-repressed gene signatures were strong features of COVID-19 BALF helper T cells. To contextualize these performances, we calculated AUCs for module scores of every gene set in Hallmark and canonical pathways curated by the Molecular Signatures Database (MSigDB) $(n=2,279$ gene sets). The top performing sets were IFN responses, as expected. The performance of the VitD-repressed gene set was within the top $1 \%$ of all gene sets (Fig. 7e and Supplementary Table 6), indicating that this was among the very best performing gene sets. We then predicted significant drugs (among $n=461$ ) potentially able to counteract genes upregulated in COVID-19 BALF helper T cells. Five of the top ten most significant drugs were steroids and two were VitD

Fig. 5 | VitD recruits keyTFs to shape transcriptional output. a, Histograms (top) and heat maps of c-JUN, VDR, STAT3 and BACH2-bound loci in carrier and VitD-treated helper T cells, centered on the peaks. $P$ values by two-sample Kolmogorov-Smirnov test comparing carrier to VitD are shown. b. Histograms of c-JUN, VDR, STAT3 and BACH2-bound loci centered on VitD-induced H3K27Ac peaks. P values by two-sample Kolmogorov-Smirnov test comparing carrier to VitD are shown. c, Proportion of genes differentially expressed after VitD treatment (DEGs from $n=3$ experiments) bound by each indicated TF. ${ }^{\star \star \star \star} P<0.0001$ by two-sided Fisher exact test compared to all genes. $\mathbf{d}$, Proportion of DEGs bound by $0,1,2,3$ or all 4 of the TFs profiled. e, Venn diagram showing overlap in TF binding between VitD DEGs. f, Heat map of DEGs showing binding of genes by c-JUN, VDR, STAT3 and BACH2. Select genes have been highlighted (right). g, Network diagram showing TF binding of genes differentially expressed after VitD treatment. Arrows join TFs to bound genes. Heat map scale indicates FC expression after VitD treatment compared to carrier. $\mathbf{h}$, Genome browser tracks at the BACH2 and IL10 loci showing H3K27Ac, c-JUN, BACH2, STAT3 and VDR binding in carrier and VitD-treated cells. Red and blue dots represent peaks in carrier and VitD-treated cells, respectively. SE denotes super-enhancer regions. Track heights are indicated on the left corner for each track. i, ChIP-qPCR for VDR or IgG in VitD-treated helper T cells from two donors. Anti-VDR ChIP fragments were probed by qPCR for enrichment of promoters of CYP24A1 (left), STAT3 (middle) and IL6 (right). Data are shown separately for each donor; bars show mean + s.e.m. of qPCR. All $P$ values are from two-sided tests. 
(alfacalcidol) (Fig. 7f and Supplementary Table 7). These were corroborated in independent datasets by GSEA, showing genes more highly expressed in lung biopsies or bulk BALF RNA-seq of COVID-19 compared to healthy control to be enriched in VitD-repressed genes (Fig. $7 \mathrm{~g}, \mathrm{~h}$ ). Significant numbers of genes modulated by VitD were shared by corticosteroid targets (Extended a
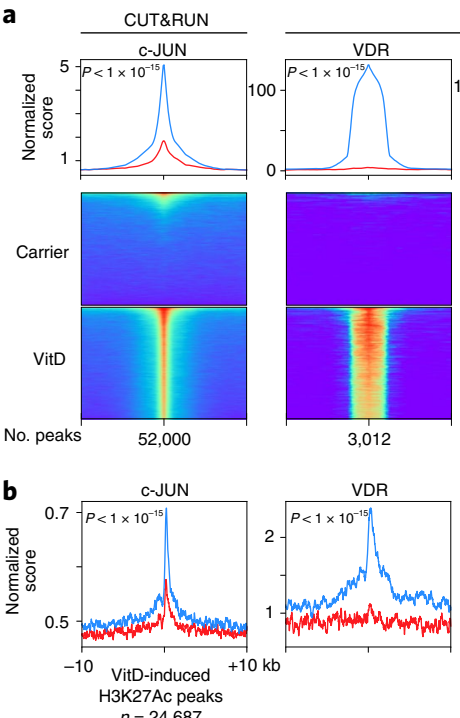

f

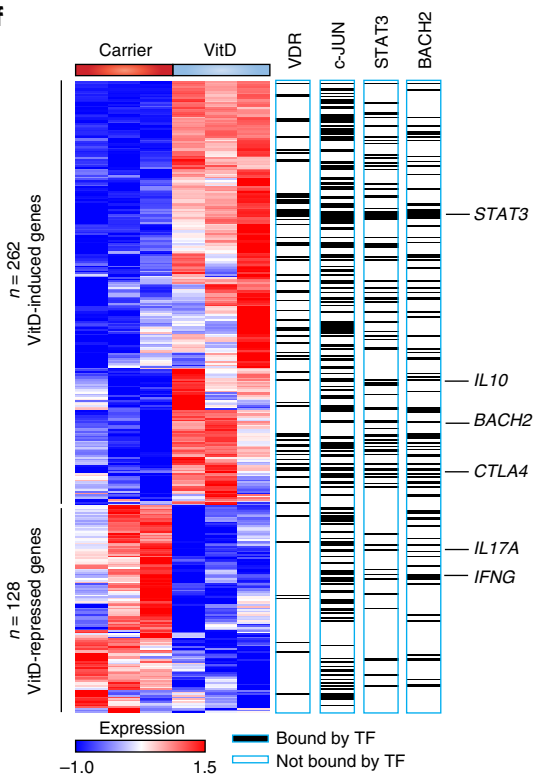

c

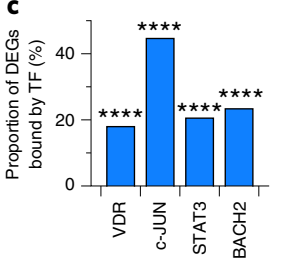

d
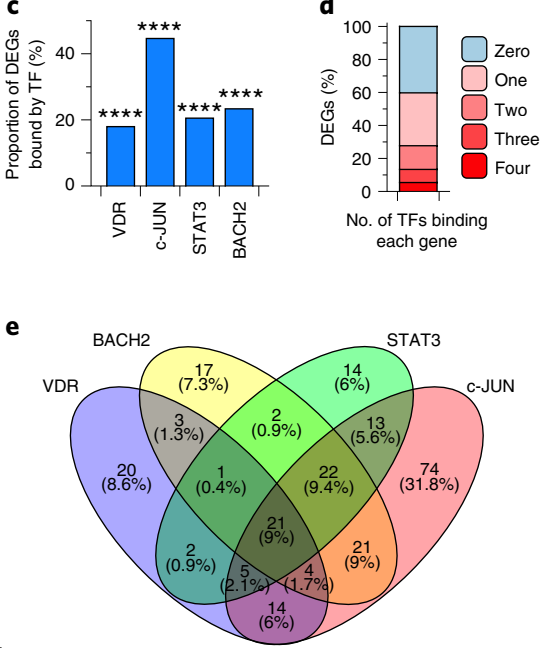

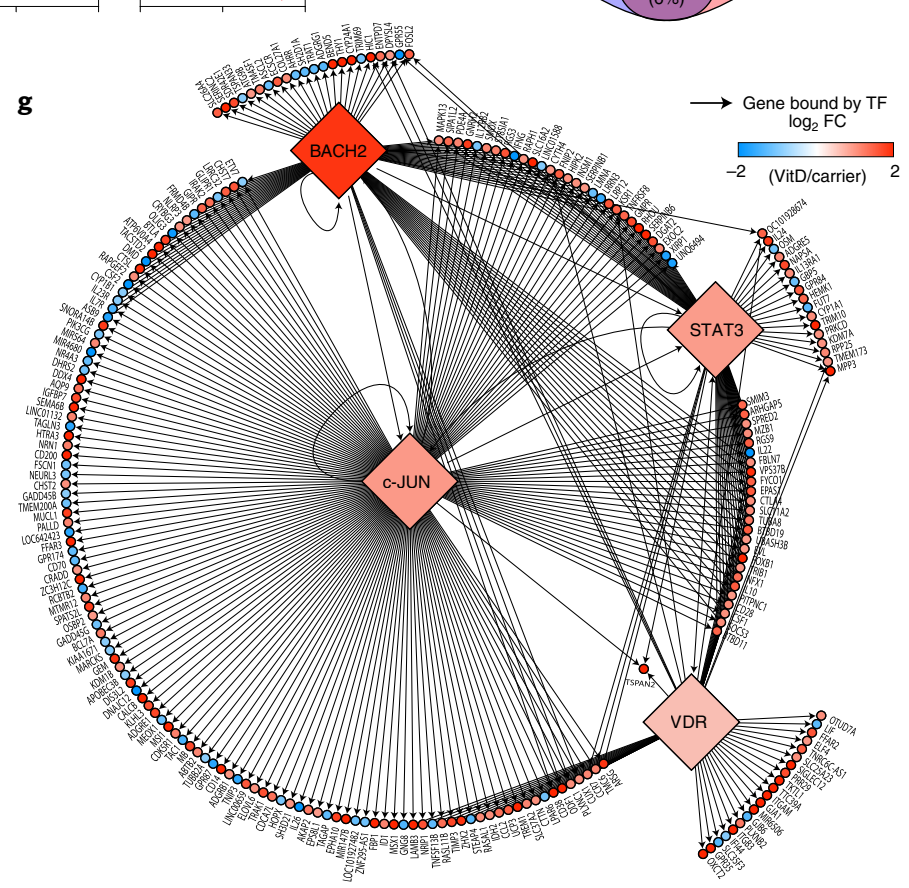

h
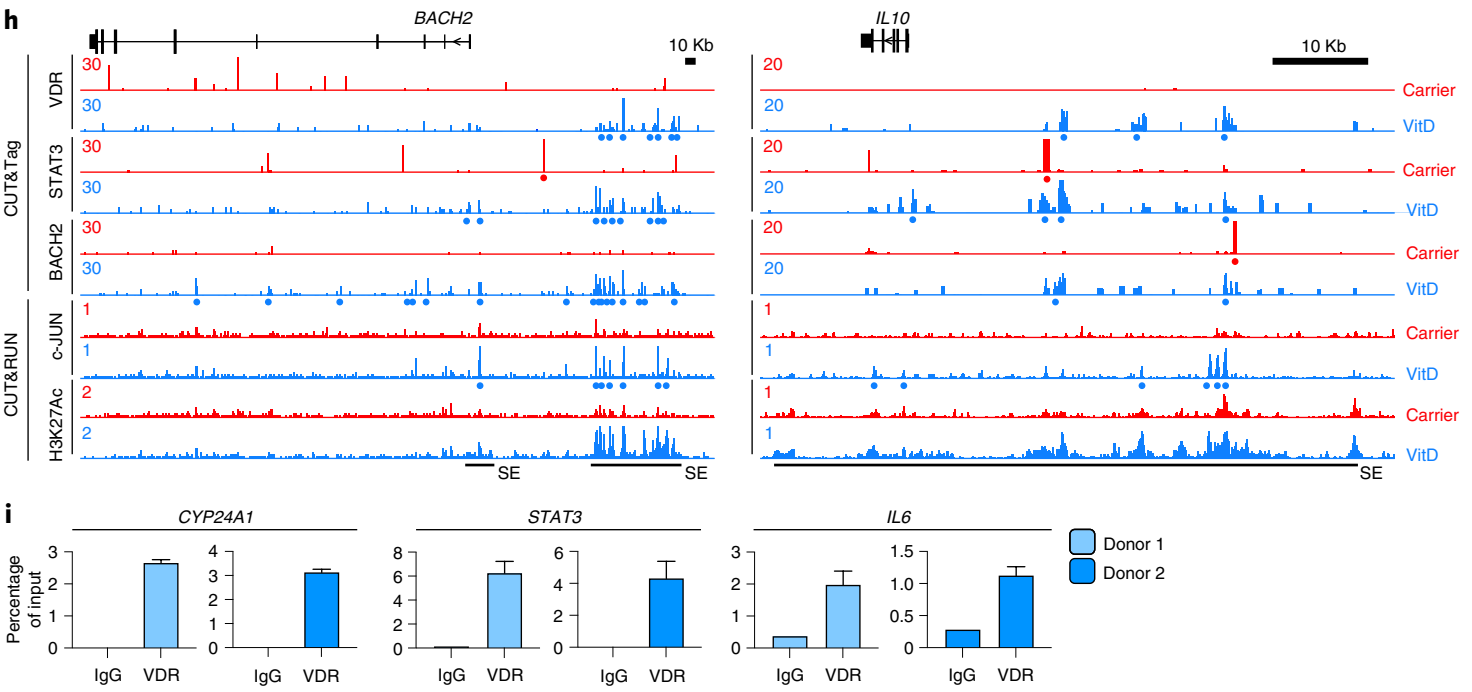

IgG VDR

IgG VDR 
Data Fig. 10c). Steroids, including dexamethasone, increase transcription of VDR so may show therapeutic synergism with VitD. Collectively, these data suggest that, in helper T cells from patients with severe COVID-19, the VitD-repressed gene set is de-repressed and that there may be clinical benefit from adding VitD to other immunomodulatory agents (Extended Data Fig. 10d).

\section{Discussion}

We showed that cell-intrinsic complement orchestrates an autocrine/paracrine autoregulatory VitD loop to initiate $\mathrm{T}_{\mathrm{H}} 1$ shutdown. VitD causes genome-wide epigenetic remodeling, induces and recruits TFs, including STAT3, c-JUN and BACH2, which collectively repress $T_{H} 1$ and $T_{H} 17$ programs and induce IL-10 via IL-6STAT3 signaling. This program is abnormal in lung helper T cells of patients with severe COVID-19, which show preferential $\mathrm{T}_{\mathrm{H}} 1$ skewing and could be potentially exploited therapeutically by using VitD as an adjunct treatment.

IFN- $\gamma$-producing airway helper $\mathrm{T}$ cells are key components of immunityto coronaviruses, including SARS-CoV1 and MERS-CoV ${ }^{20}$. $\mathrm{T}_{\mathrm{H}} 1$-polarized responses are also a feature of SARS-CoV2 in humans $^{24}$ and severe COVID-19 is accompanied by prolonged, exacerbated, circulating $\mathrm{T}_{\mathrm{H}} 1$ responses ${ }^{21}$. Complement receptor signaling is a driver of $\mathrm{T}_{\mathrm{H}} 1$ differentiation and required for effective antiviral responses ${ }^{12,42}$. C3 cleavage generates $\mathrm{C} 3 \mathrm{~b}$, which binds CD46 on T cells. We have previously shown that the lungs in COVID-19 are a complement-rich microenvironment, that local $\mathrm{CD}^{+} \mathrm{T}$ lymphocytes have a CD46-activated signature ${ }^{13}$ and show here that these $\mathrm{T}$ cells are $\mathrm{T}_{\mathrm{H}} 1$-polarized. Pro-inflammatory function is important for pathogen clearance, but a switch into IL-10 production is a natural component during successful transition into the $\mathrm{T}_{\mathrm{H}} 1$ shutdown program and reduces collateral damage ${ }^{16}$. Inability to produce IL-10 results in more efficient clearance of infections but severe tissue damage from uncontrolled $\mathrm{T}_{\mathrm{H}} 1$ responses results in death ${ }^{2}$. The benefits of remediating inflammatory pathways in severe COVID-19 is demonstrated by successful trials of dexamethasone, an immunosuppressive drug that reduces mortality ${ }^{1}$.

VitD has pleiotropic functions in the immune system, including antimicrobial as well as regulatory properties, which are cell- and context-dependent ${ }^{43,44}$. VitD deficiency is associated with higher prevalence and worse outcomes from infections, including influenza, tuberculosis and viral upper respiratory tract illnesses ${ }^{17}$, as well as autoimmune diseases, including type 1 diabetes, multiple sclerosis, rheumatoid arthritis and inflammatory bowel disease ${ }^{18}$. Helper T cells play key roles in all these diseases. Thus, understanding VitD biology in helper T cells has potential translational impact.

Our data indicate the complexities of TFs working within networks to regulate sets of genes. After VitD ligates VDR, c-JUN,
STAT3 and BACH2 are recruited to acetylated loci, shaping the transcriptional response to VitD. c-JUN is a member of the AP-1 basic leucine zipper family, primarily involved in DNA transcription $^{45}$. This TF was bound adjacent to $40 \%$ of VitD-regulated coding loci. $\mathrm{BACH} 2$ is a critical immunoregulatory $\mathrm{TF}^{38,39}$ downregulated in lesional versus non-lesional psoriatic skin ${ }^{46}$. Active VitD concentrations inversely correlate with severity of psoriasis ${ }^{40}$. We found that VitD treatment of psoriatic lesional skin upregulated $\mathrm{BACH} 2$ expression. Both haploinsufficiency and single nucleotide variants of $\mathrm{BACH} 2$ associate with monogenic and polygenic autoimmunity, respectively, in humans ${ }^{36,47}$. No BACH2 knockout humans have yet been identified, suggesting incompatibility of complete BACH2 deficiency with life. Indeed, Bach $2^{-l-}$ mice succumb to fatal autoimmunity. We found that loss of even $50 \%$ of the normal cellular concentration of $\mathrm{BACH} 2$ in the haploinsufficient state substantially altered ( $70 \%$ of) the VitD-regulated transcriptome. As only a proportion of these genes were directly $\mathrm{BACH} 2$-bound, it is probable that $\mathrm{BACH} 2$ is a requisite for normal recruitment and function of the other transcriptional regulators.

Both the incidence and severity of COVID-19 are epidemiologically associated with VitD deficiency/insufficiency ${ }^{19}$, but the molecular mechanisms remain unknown. We found a link between the inflammatory $\mathrm{T}_{\mathrm{H}} 1$ program and a VitD-repressed gene set. Attempts to study $\mathrm{CD} 4^{+} \mathrm{T}$ cells from the site of inflammation were unsuccessful due to the rapid apoptosis of patient cells, but our in silico analyses suggest either dysregulation of the VitD program in COVID-19 or that simple deficiency/insufficiency of substrate (VitD) might explain the epidemiological association.

IL-6 is a pleiotropic, often pro-inflammatory, cytokine. IL-6 is implicated in the COVID-19 'cytokine storm' and targeting of this cytokine specifically has proved beneficial to patients ${ }^{32}$. Our data suggest that pro-inflammatory IL-6 functions may be redirected to production of anti-inflammatory IL-10 by VitD in activated human helper $\mathrm{T}$ cells. VitD supplementation in children significantly increases serum IL-6 (and nonsignificantly increases IL-10) ${ }^{48}$ indicating that these observations may also occur in vivo. In the skin, where VitD concentrations are high, IL-6 overexpression protects from injurious stimuli or infection ${ }^{49}$ and IL-6-deficiency impairs wound healing ${ }^{50}$. Moreover, an adverse effect of anti-IL-6R for treating inflammatory arthritis is idiosyncratic development of psoriasis $^{51}$, indicating a tolerogenic role for IL-6 at this site. Thus, adjunct VitD therapy in severe COVID-19 could potentially divert pro-inflammatory and induce anti-inflammatory effects of IL-6, which may be an alternative to blocking IL-6R signaling.

These data identified the VitD pathway as a potential mechanism to accelerate shutdown of $\mathrm{T}_{\mathrm{H}} 1$ cells in severe COVID-19. From experience in other diseases, it is likely that VitD will be ineffective

Fig. 6 | BACH2 is an important regulator of the VitD response in helper T cells. a, BACH2 Immunoblot in VitD- and carrier-treated helper T cell lysates. Shown is a representative example from $n=3$ experiments. $\mathbf{b}-\mathbf{e}$, Representative dermal images of lesional skin from psoriasis patients with $(n=2)$ and without $(n=3)$ VitD supplementation stained for BACH2 (red) and CD3 (green), showing overview (left-most) and zoomed images (right three images) (b), number of nuclei per image (c), percentage of $\mathrm{BACH} 2^{+}$cells relative to nuclei frequency/image (d) and average number of $\mathrm{BACH} 2 \mathrm{foci}$ per cell (e). For c-e, 5-6 images were acquired for each sample and are shown as median values, with minimum, maximum, 25\% and $75 \%$ quartiles. $\mathbf{f , g}$, Pie charts comparing percentage of VitD-regulated genes bound and regulated by mouse (f) or human BACH2 ( $\mathbf{g}$ ) against all genes in the genome. The two-sided Fisher exact $P$ value is shown. $\mathbf{h}$, GSEA showing enrichment in VitD-induced genes comparing transcriptomes of VitD-treated wild-type control $\left(B A C H 2^{W T / W T}\right.$ ) with BACH2-haploinsufficient helper T cells (BACH2 ${ }^{W T / 24 P}$ ). Shown is the empirical $P$ value; NES, normalized enrichment score. $\mathbf{i}$, Sankey diagram showing the relationship between genes up- and downregulated by VitD in BACH2 BACH2WT/L24P compared to BACH2 ${ }^{\text {WT/WT }}$ helper T cells and the binding of those genes by c-JUN, VDR, BACH2 and STAT3. j, Pie charts comparing percentage of genes up- and downregulated by VitD in BACH2 sufficient cells that are differently regulated in $\mathrm{BACH} 2$ haploinsufficiency. The percentage of those genes bound by $\mathrm{BACH} 2$ are shown underneath. $\mathbf{k}$, Top three enriched MSigDB canonical pathways pertaining to the genes bound by BACH2 and differently regulated in BACH2-haploinsufficient cells. Indicated are three relevant genes contributing to enriched pathways. I, Heat map showing expression patterns of select VitD-regulated genes in the transcriptomes of carrier- and VitD-treated BACH2 ${ }^{\mathrm{WT} / \mathrm{WT}}$ and BACH2 ${ }^{\mathrm{WT} / L 24 \mathrm{P}}$ helper T cells. $\mathbf{m}$, Genome browser tracks at IL6R locus showing H3K27Ac, c-JUN, BACH2, STAT3 and VDR binding in carrier and VitD-treated cells. Red and blue dots represent peaks in carrier and VitD-treated cells, respectively. Track heights are indicated (left). In vitro T cell experiments depicted were activated with anti-CD3+ anti-CD28. NS, not significant; ${ }^{\star} P<0.01$; ${ }^{\star \star \star \star} P<0.0001$ by unpaired two-tailed $t$-test. 
as monotherapy. Combination therapy could potentially ameliorate significant adverse effects of other drugs, for example high-dose corticosteroids, including over-immunosuppression or metabolic side-effects. An important consideration of VitD therapy in COVID-19 is stimulation of IL-6 production from $\mathrm{CD}^{+} \mathrm{T}$ cells.
Although autocrine/paracrine IL-6 induces IL-10 in these cells, IL-6 could potentially have pro-inflammatory properties on other cells. These possibilities may be mitigated by adding VitD as an adjunct to other immunomodulators, such as corticosteroids or JAK inhibitors ${ }^{13}$. Of note, two randomized clinical trials with

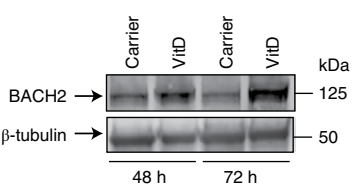

$\mathbf{f}$

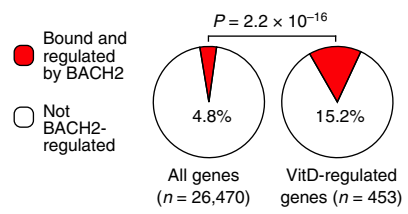

$\mathbf{g}$

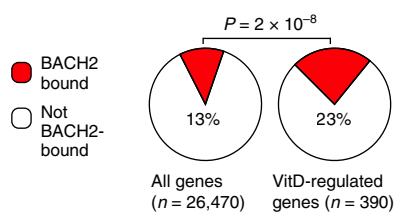

h

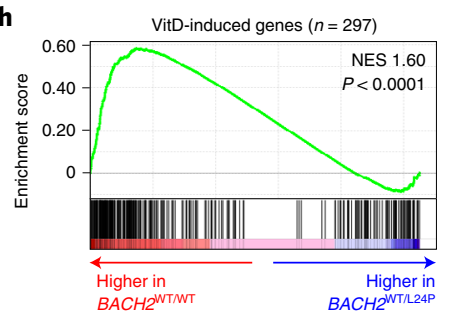

j

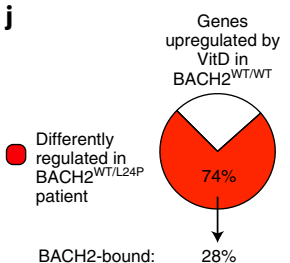

BACH2-bound:

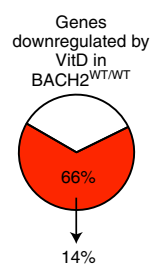

I

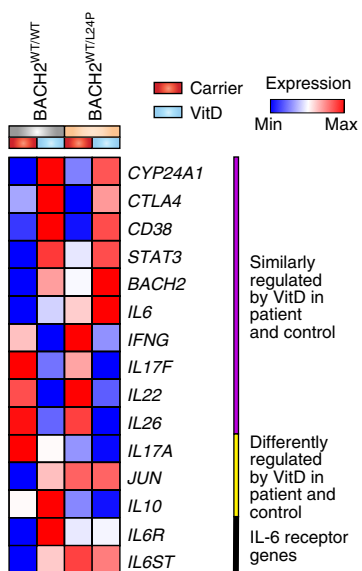

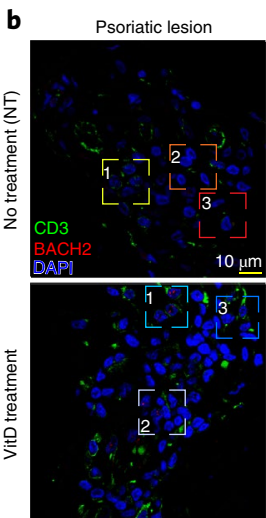
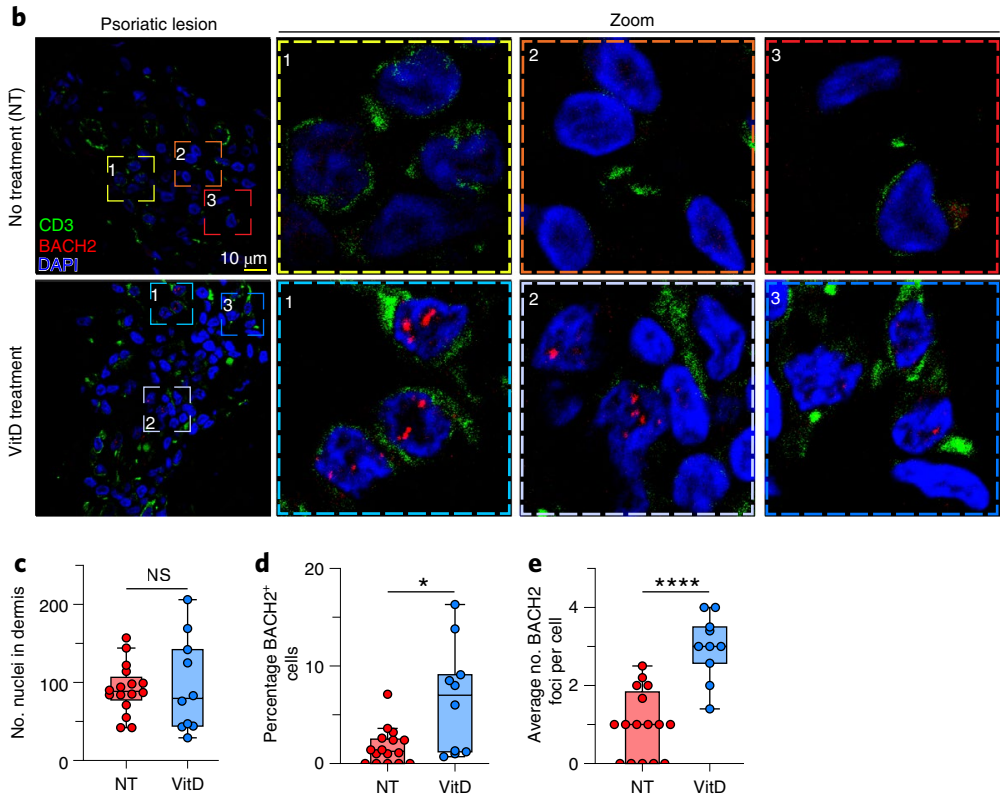

i

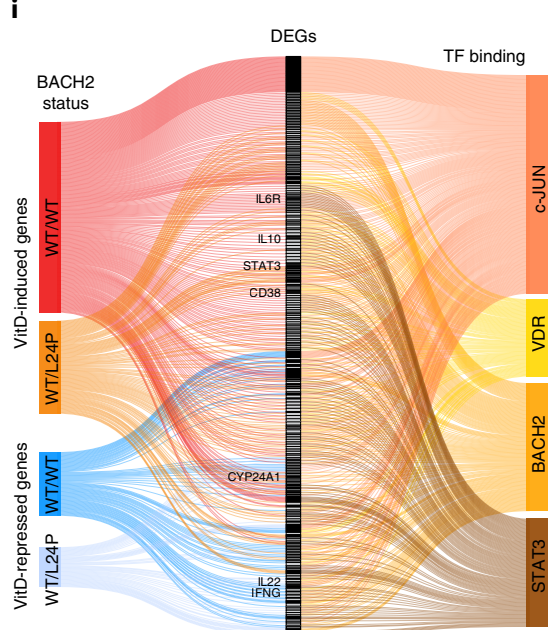

$\mathbf{k}$
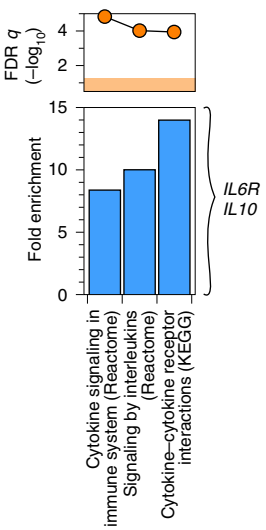

m

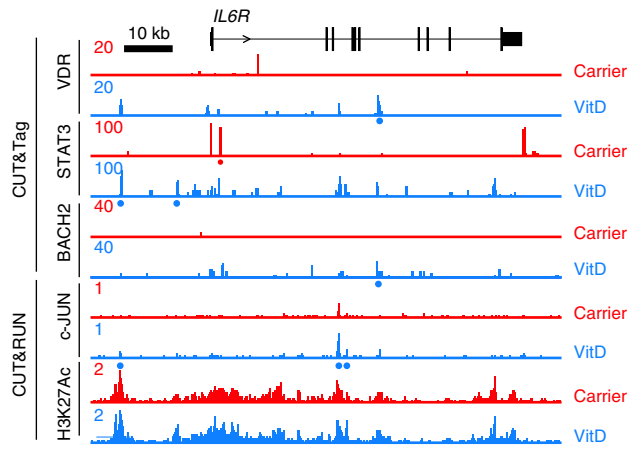



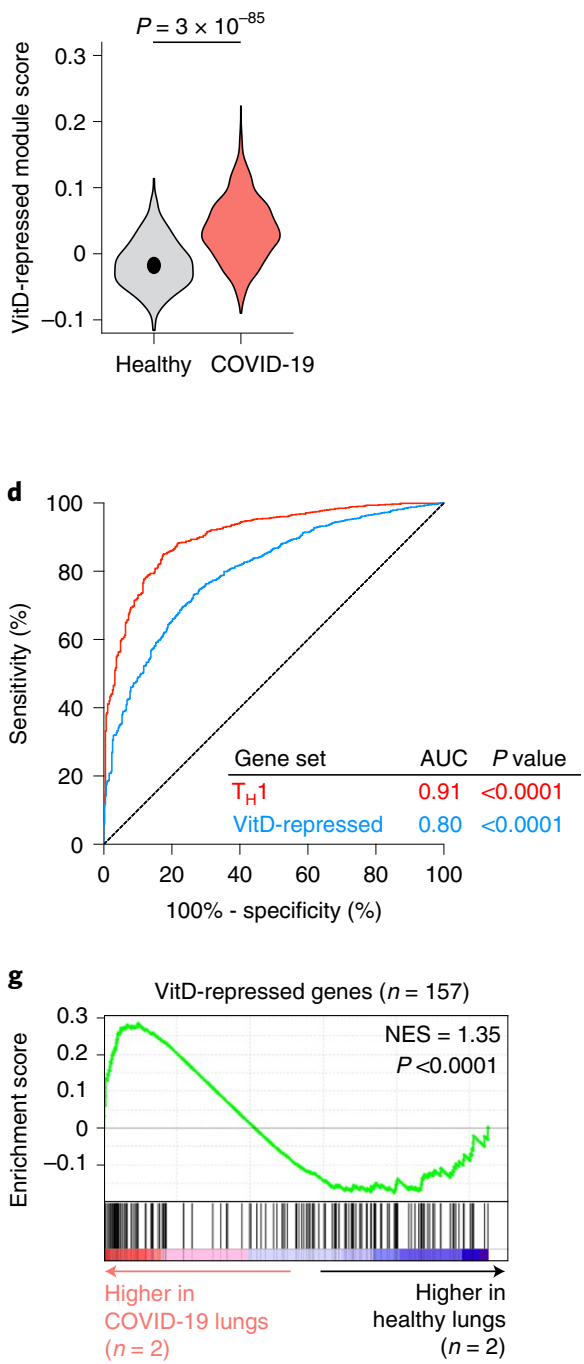
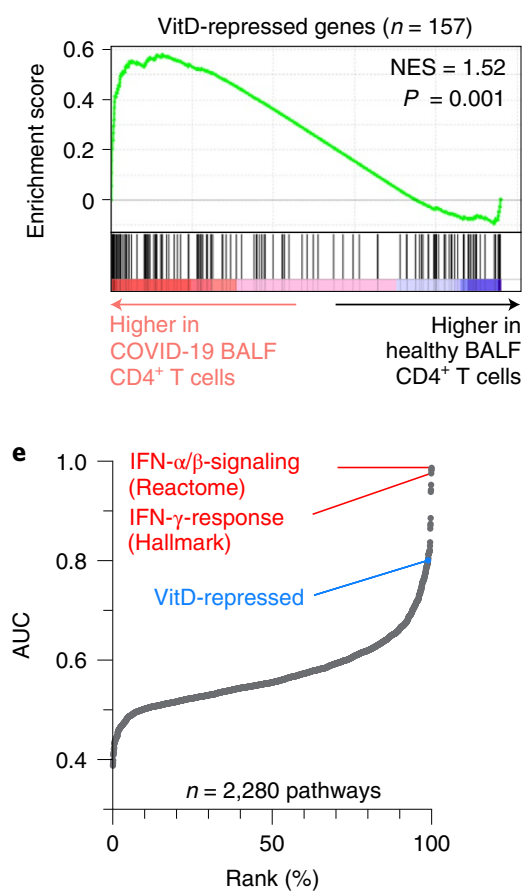

h

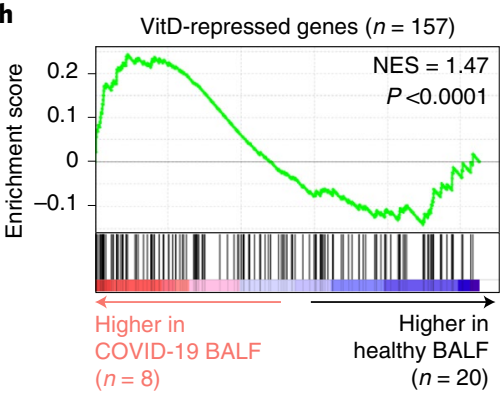

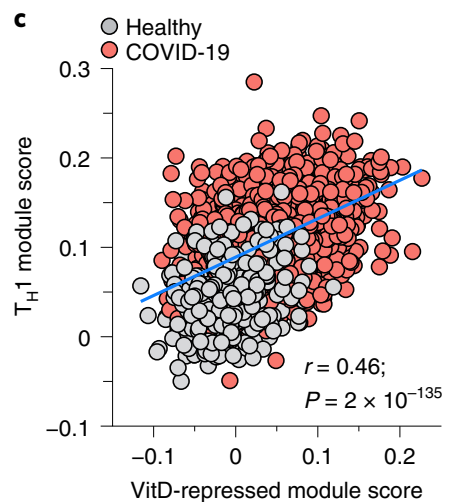

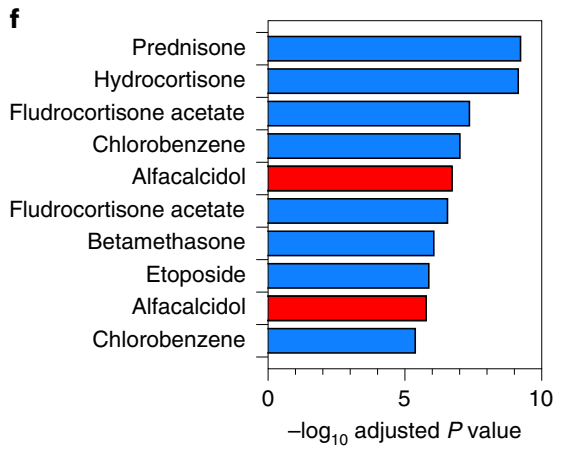

Fig. 7 | VitD is among the top predicted therapeutics to retract the $\mathbf{T}_{\mathrm{H}} \mathbf{1}$ program in SARS-CoV2-infected lungs. a, Violin plots showing expressions of VitD-repressed genes, summarized as module scores, in BALF helper T cells of patients with COVID-19 and healthy controls. Exact $P$ values in a have been calculated using two-tailed Wilcoxon tests. b, GSEA showing enrichment in VitD-repressed genes within genes more highly expressed in scRNA-seq CD4+ BALF T cells of patients with COVID-19 compared to healthy controls. c, Correlation between module scores of $\mathrm{T}_{H} 1$ genes and $\mathrm{VitD}$-repressed genes on a per-cell basis in BALF helper T cells of patients with COVID-19 and healthy controls. Pearson $r$ and exact $P$ values are shown. d, ROC curve, evaluating the performance of the $T_{H} 1$ and VitD-repressed module scores to distinguish BALF helper T cells of patients with COVID-19 from healthy controls. Shown are the AUC statistics and $P$ values. e, Analyses showing the performance of all MSigDB canonical and Hallmark gene sets to distinguish BALF helper T cells of patients with COVID-19 from healthy controls, ranked by AUC values. Marked are the top two performing gene sets in red and the position of the VitD-repressed gene set within the top $1 \%$ of all gene sets. f, Top ten drugs predicted (out of 461 significant drugs) to counteract genes induced in BALF helper T cells of patients with COVID-19 compared to healthy controls, ordered by adjusted $P$ value. Highlighted in red is alfacalcidol, a US Food and Drug Administration-approved active form of VitD. $\mathbf{g}, \mathbf{h}$, GSEA showing enrichment in VitD-repressed genes for genes more highly expressed in bulk RNA-seq lung biopsy specimens ( $\mathbf{g}$ ) and bulk RNA-seq BALF cells (h) of COVID-19 compared to healthy controls. Empirical $P$ values are shown for GSEA in $\mathbf{b}, \mathbf{g}$, $\mathbf{h}$. $P$ values in $\mathbf{d}$ are from the Mann-Whitney $U$-statistic. Data in a-f are from $n=8$ patients with COVID-19 and $n=3$ healthy controls, sourced from GSE145926 and GSE122960. Data in $\mathbf{g}, \mathbf{h}$ are from GSE147507 and HRA000143, respectively and $n$ numbers are indicated.

calcifediol, a VitD analog with high bioavailability not requiring hepatic 25-hydroxylation, comprising $>1,000$ patients together, reported reductions in risk of intensive-care unit admission or death when used in addition to standard care (odds ratio of 0.13 and 0.22 , respectively ${ }^{52,53}$ ). These findings are not necessarily specific to COVID-19, as VitD can protect against acute respiratory tract infections in general ${ }^{17}$.

In conclusion, we identified an autocrine/paracrine VitD loop permitting $\mathrm{T}_{\mathrm{H}} 1$ cells to both activate and respond to VitD as part of a shutdown program repressing IFN- $\gamma$ and enhancing IL-10.
These events involved significant epigenetic reshaping and recruitment of a network of key TFs. These pathways could potentially be exploited therapeutically to accelerate the shutdown program of hyper-inflammatory cells in patients with severe COVID-19.

\section{Online content}

Any methods, additional references, Nature Research reporting summaries, source data, extended data, supplementary information, acknowledgements, peer review information; details of author contributions and competing interests; and statements of 
data and code availability are available at https://doi.org/10.1038/ s41590-021-01080-3.

Received: 23 July 2020; Accepted: 26 October 2021;

Published online: 11 November 2021

\section{References}

1. RECOVERY Collaborative Group et al.Dexamethasone in hospitalized patients with COVID-19. New Engl. J. Med. 384, 693-704 (2020).

2. Gazzinelli, R. T. et al. In the absence of endogenous IL-10, mice acutely infected with Toxoplasma gondii succumb to a lethal immune response dependent on $\mathrm{CD}^{+} \mathrm{T}$ cells and accompanied by overproduction of IL-12, IFN- $\gamma$ and TNF- $\alpha$. J. Immunol. 157, 798-805 (1996).

3. Noris, M. \& Remuzzi, G. Overview of complement activation and regulation. Semin. Nephrol. 33, 479-492 (2013).

4. Merle, N. S., Church, S. E., Fremeaux-Bacchi, V. \& Roumenina, L. T. Complement system part I - molecular mechanisms of activation and regulation. Front. Immunol. 6, 262 (2015).

5. Robbins, R. A., Russ, W. D., Rasmussen, J. K. \& Clayton, M. M. Activation of the complement system in the adult respiratory distress syndrome 1-4. Am. Rev. Respir. Dis. 135, 651-658 (1987).

6. Ohta, R. et al. Serum concentrations of complement anaphylatoxins and proinflammatory mediators in patients with $2009 \mathrm{H} 1 \mathrm{~N} 1$ influenza. Microbiol. Immunol. 55, 191-198 (2011).

7. Carvelli, J. et al. Association of COVID-19 inflammation with activation of the C5a-C5aR1 axis. Nature https://doi.org/10.1038/s41586-020-2600-6 (2020).

8. Sinkovits, G. et al. Complement overactivation and consumption predicts in-hospital mortality in SARS-CoV-2 infection. Front. Immunol. 12, 663187 (2021).

9. Ramlall, V. et al. Immune complement and coagulation dysfunction in adverse outcomes of SARS-CoV-2 infection. Nat. Med. 26, 1609-1615 (2020).

10. Rockx, B. et al. Early upregulation of acute respiratory distress syndrome-associated cytokines promotes lethal disease in an aged-mouse model of severe acute respiratory syndrome coronavirus infection. J. Virol. 83, 7062-7074 (2009).

11. Mastaglio, S. et al. The first case of COVID-19 treated with the complement C3 inhibitor AMY-101. Clin. Immunol. 215, 108450 (2020).

12. Liszewski, M. K. et al. Intracellular complement activation sustains $\mathrm{T}$ cell homeostasis and mediates effector differentiation. Immunity $\mathbf{3 9}$, 1143-1157 (2013).

13. Yan, B. et al. SARS-CoV-2 drives JAK1/2-dependent local complement hyperactivation. Sci. Immunol. 6, eabg0833 (2021).

14. West, E. E., Kolev, M. \& Kemper, C. Complement and the regulation of T cell responses. Annu Rev. Immunol. 36, 309-338 (2018).

15. Break, T. J. et al. Aberrant type 1 immunity drives susceptibility to mucosal fungal infections. Science 371, eaay5731 (2021).

16. Cardone, J. et al. Complement regulator CD46 temporally regulates cytokine production by conventional and unconventional T cells. Nat. Immunol. 11, 862-871 (2010).

17. Yamshchikov, A., Desai, N., Blumberg, H., Ziegler, T. \& Tangpricha, V. Vitamin D for treatment and prevention of infectious diseases: a systematic review of randomized controlled trials. Endocr. Pr. 15, 438-449 (2009).

18. Holick, M. F. Vitamin D deficiency. N. Engl. J. Med. 357, 266-281 (2007).

19. Akbar, M. R., Wibowo, A., Pranata, R. \& Setiabudiawan, B. Low serum 25-hydroxyvitamin D (vitamin D) level is associated with susceptibility to COVID-19, severity, and mortality: a systematic review and meta-analysis. Front. Nutr. 8, 660420 (2021).

20. Zhao, J. et al. Airway memory $\mathrm{CD} 4\left(^{+}\right) \mathrm{T}$ cells mediate protective immunity against emerging respiratory coronaviruses. Immunity 44, 1379-1391 (2016).

21. Lucas, C. et al. Longitudinal analyses reveal immunological misfiring in severe COVID-19. Nature 584, 463-469 (2020).

22. Kolev, M. et al. Diapedesis-induced integrin signaling via LFA-1 facilitates tissue immunity by inducing intrinsic complement $\mathrm{C} 3$ expression in immune cells. Immunity 52, 513-527 (2020)

23. Magro, C. et al. Complement-associated microvascular injury and thrombosis in the pathogenesis of severe COVID-19 infection: a report of five cases. Transl. Res. 220, 1-13 (2020).

24. Grifoni, A. et al. Targets of T cell responses to SARS-CoV-2 coronavirus in humans with COVID-19 disease and unexposed individuals. Cell 181, 1489-1501 (2020).

25. Sigmundsdottir, H. et al. DCs metabolize sunlight-induced vitamin D3 to 'program' T cell attraction to the epidermal chemokine CCL27. Nat. Immunol. 8, 285-293 (2007)

26. Veldman, C. M., Cantorna, M. T. \& DeLuca, H. F. Expression of 1,25-dihydroxyvitamin D3 receptor in the immune system. Arch. Biochem. Biophys. 374, 334-338 (2000)
27. Chen, D.-J. et al. Altered microRNAs expression in T cells of patients with SLE involved in the lack of vitamin D. Oncotarget 8, 62099-62110 (2017).

28. Jeffery, L. E. et al. 1,25-Dihydroxyvitamin D3 and IL-2 combine to inhibit $\mathrm{T}$ cell production of inflammatory cytokines and promote development of regulatory T cells expressing CTLA-4 and FoxP3. J. Immunol. 183, 5458-5467 (2009).

29. Barrat, F. J. et al. In vitro generation of interleukin 10-producing regulatory $\mathrm{CD}^{+} \mathrm{T}$ cells is induced by immunosuppressive drugs and inhibited by $\mathrm{T}$ helper type $1\left(\mathrm{~T}_{\mathrm{H}} 1\right)$ - and $\mathrm{T}_{\mathrm{H}} 2$-inducing cytokines. J. Exp. Med. 195, 603-616 (2002).

30. Gagliani, N. et al. Coexpression of CD49b and LAG-3 identifies human and mouse T regulatory type 1 cells. Nat. Med. 19, 739-746 (2013).

31. Hunter, C. A. \& Jones, S. A. IL-6 as a keystone cytokine in health and disease. Nat. Immunol. 16, 448-457 (2015).

32. Abani, O. et al. Tocilizumab in patients admitted to hospital with COVID-19 (RECOVERY): a randomised, controlled, open-label, platform trial. Lancet 397, 1637-1645 (2021).

33. Stumhofer, J. S. et al. Interleukins 27 and 6 induce STAT3-mediated T cell production of interleukin 10. Nat. Immunol. 8, 1363-1371 (2007).

34. Jin, J.-O., Han, X. \& Yu, Q. Interleukin-6 induces the generation of IL-10-producing $\operatorname{Tr} 1$ cells and suppresses autoimmune tissue inflammation. J. Autoimmun. 40, 28-44 (2013).

35. Bettelli, E. et al. Reciprocal developmental pathways for the generation of pathogenic effector $\mathrm{T}_{\mathrm{H}} 17$ and regulatory $\mathrm{T}$ cells. Nature 441, 235-238 (2006).

36. Afzali, B. et al. BACH2 immunodeficiency illustrates an association between super-enhancers and haploinsufficiency. Nat. Immunol. 18, 813-823 (2017).

37. Whyte, W. A. et al. Master transcription factors and mediator establish super-enhancers at key cell identity genes. Cell 153, 307-319 (2013).

38. Roychoudhuri, R. et al. BACH2 represses effector programs to stabilize T(reg)-mediated immune homeostasis. Nature 498, 506-510 (2013).

39. Povoleri, G. A. M. et al. Human retinoic acid-regulated $\mathrm{CD} 161^{+}$regulatory $\mathrm{T}$ cells support wound repair in intestinal mucosa. Nat. Immunol. 19, 1403-1414 (2018).

40. Playford, M. P. et al. Serum active 1,25(OH)2D, but not inactive $25(\mathrm{OH}) \mathrm{D}$ vitamin D levels are associated with cardiometabolic and cardiovascular disease risk in psoriasis. Atherosclerosis 289, 44-50 (2019).

41. Armstrong, A. W. \& Read, C. Pathophysiology, clinical presentation, and treatment of psoriasis. JAMA 323, 1945-1960 (2020).

42. Ghannam, A., Fauquert, J.-L., Thomas, C., Kemper, C. \& Drouet, C. Human complement $\mathrm{C} 3$ deficiency: $\mathrm{T}_{\mathrm{H}} 1$ induction requires $\mathrm{T}$ cell-derived complement C3a and CD46 activation. Mol. Immunol. 58, 98-107 (2014).

43. Schedel, M. et al. $1,25 \mathrm{D} 3$ prevents $\mathrm{CD} 8^{+} \mathrm{Tc} 2$ skewing and asthma development through VDR binding changes to the Cypllal promoter. Nat. Commun. 7, 10213 (2016).

44. Song, J. et al. Vitamin D receptor restricts T helper 2-biased inflammation in the heart. Cardiovasc. Res. 114, 870-879 (2018).

45. Bohmann, D. et al. Human proto-oncogene c-jun encodes a DNA binding protein with structural and functional properties of transcription factor AP-1. Science 238, 1386-1392 (1987).

46. Tian, S. et al. Meta-analysis derived (MAD) transcriptome of psoriasis defines the 'core' pathogenesis of disease. PLoS ONE 7, e44274 (2012).

47. Franke, A. et al. Genome-wide meta-analysis increases to 71 the number of confirmed Crohn's disease susceptibility loci. Nat. Genet. 42, $1118-1125$ (2010).

48. Yegorov, S., Bromage, S., Boldbaatar, N. \& Ganmaa, D. Effects of vitamin D supplementation and seasonality on circulating cytokines in adolescents: analysis of data from a feasibility trial in Mongolia. Front. Nutr. 6, 166 (2019).

49. Turksen, K., Kupper, T., Degenstein, L., Williams, I. \& Fuchs, E. Interleukin 6: insights to its function in skin by overexpression in transgenic mice. Proc. Natl Acad. Sci. USA 89, 5068-5072 (1992).

50. Lin, Z., Kondo, T., Ishida, Y., Takayasu, T. \& Mukaida, N. Essential involvement of IL-6 in the skin wound-healing process as evidenced by delayed wound healing in IL-6-deficient mice. J. Leukoc. Biol. 73, 713-721 (2003)

51. Wendling, D., Letho-Gyselinck, H., Guillot, X. \& Prati, C. Psoriasis onset with tocilizumab treatment for rheumatoid arthritis. J. Rheumatol. 39, 657-657 (2012).

52. Nogues, X. et al. Calcifediol treatment and COVID-19-related outcomes. J. Clin. Endocrinol. Metab. 106, dgab405 (2021).

53. Alcala-Diaz, J. F. et al. Calcifediol treatment and hospital mortality due to COVID-19: a cohort study. Nutrients 13, 1760 (2021).

54. Kolev, M. et al. Complement regulates nutrient influx and metabolic reprogramming during $\mathrm{T}_{\mathrm{H}} 1$ cell responses. Immunity 42, 1033-1047 (2015).

Publisher's note Springer Nature remains neutral with regard to jurisdictional claims in published maps and institutional affiliations.

This is a U.S. government work and not under copyright protection in the U.S.; foreign copyright protection may apply 2021 


\section{Methods}

Ethics approvals. Human studies, conducted in accordance with the Declaration of Helsinki, were approved by the Institutional Review Board of Guy's Hospital (reference 09/H0707/86), National Institutes of Health (NIH) (approval nos. 7458, PACI, 13-H-0065 and 00-I-0159) and Imperial College London (approval no. 12/WA/0196 ICHTB HTA license number 12275 to project R14098). All patients provided informed written consent.

Human T cell isolation and culture. Human PBMCs were purified from anonymized leukodepletion cones (Blood Transfusion Service, NHS Blood and Transplantation) or healthy volunteer whole blood packs/buffy coats from the NIH Blood Bank and from fresh patient blood. Leukodepletion cones were diluted 1:4 with PBS and layered onto Lymphoprep (Axis-Shield). Whole blood packs/buffy coats were diluted 1:2 with PBS onto Lymphocyte Separation Medium (25-072-CV LSM, Corning) and centrifuged.

$\mathrm{CD} 4^{+} \mathrm{CD} 25^{-}$cells were used throughout, unless specified. $\mathrm{CD} 4^{+} \mathrm{T}$ cells were enriched using RosetteSep Human $\mathrm{CD}^{+}$enrichment cocktail (StemCell) with $\mathrm{CD} 4{ }^{+} \mathrm{CD} 25^{-}$cells obtained by depletion of CD25 $5^{+} \mathrm{T}$ cells using CD25-positive selection (CD25 microbeads II, Human, Miltenyi Biotec) followed by unlabeled cell collection. Human memory $\mathrm{CD}^{+} \mathrm{T}$ cells were used for RNA-seq, CUT\&RUN and CUT\&Tag experiments as these cells express the VDR. These cells were isolated from PBMCs using Miltenyi Memory CD4 ${ }^{+} \mathrm{T}$ cell Isolation Kit Human (130091893) or StemCell EasySep Human Memory CD4 ${ }^{+}$T Cell Enrichment kit (19157) or by flow sorting.

For flow sorting, bulk $\mathrm{CD} 4^{+} \mathrm{T}$ cells were enriched as above then stained with antibodies against CD4 (OKT4, Thermo Fisher Scientific), CD45RA (HI100, BioLegend), CD45RO (UCLH1, BD Biosciences) and CD25 (2A3, BD Biosciences) in MACS buffer at $4^{\circ} \mathrm{C}$ for $30 \mathrm{~min}$. CD $4^{+} \mathrm{CD} 25^{-} \mathrm{CD} 45 \mathrm{RO}^{+} \mathrm{CD} 45 \mathrm{RA}^{-}$memory cells were sorted into medium to $>99 \%$ purity by a FACSAria (BD Biosciences). Sorting strategy and representative post-sort purities are shown in Extended Data Fig. 10e.

Cells were cultured in X-VIVO-15 Serum-free Hematopoietic Cell Medium (04-418Q, Lonza) supplemented with $50 \mathrm{IU} \mathrm{ml}^{-1}$ penicillin, $50 \mu \mathrm{g} \mathrm{ml}^{-1}$ streptomycin and $2 \mathrm{mM}$ L-glutamine (Gibco), at $37^{\circ} \mathrm{C} 5 \% \mathrm{CO}_{2}$ in a non-tissue culture-treated 96-well U-bottom plate (Greiner) at a density of $10^{6}$ cells $\mathrm{ml}^{-1}$ in $200 \mu$ l. Cells were activated using Human T-Activator CD3/CD28 Dynabeads (11131D, Gibco, cell to bead ratio of $4: 1$ ). Where indicated, cells were plate-activated in non-tissue culture-treated 48-well plates (Greiner) pre-coated with anti-CD3 (OKT3, from Washington University hybridoma facility), anti-CD3 + anti-CD28 (CD28.2, Becton Dickinson) or anti-CD3 + anti-CD46 (TRA-2-10, a gift from J. P. Atkinson, Washington University), with or without $1 \mu \mathrm{M}$ cathepsin L inhibitor (ALX-260133-M001, Enzo Life Sciences), with IL-2 included (50 $\mathrm{U} \mathrm{ml}^{-1}$; PeproTech), in a total volume of $250 \mu \mathrm{l}$ at a density of $5 \times 10^{5}$ per well. Antibody-coated plates were prepared by diluting antibodies $\left(2 \mu \mathrm{g} \mathrm{ml}^{-1}\right)$ in $\mathrm{D}-\mathrm{PBS}+\mathrm{Ca}^{2+} \mathrm{Mg}^{2+}$ (Gibco) and adding to 48 -well plates overnight in a volume of $150 \mu \mathrm{l}$ per well at $4{ }^{\circ} \mathrm{C}$, subsequently plates were rinsed with PBS before cell suspension addition and centrifugation (swinging-bucket rotor, $300 \mathrm{~g}, 3 \mathrm{~min}$, room temperature). Cells were additionally cultured with 1 $\alpha, 25$-dihydroxyvitamin D3 (BML-DM200-0050, Enzo Life Sciences) or 25-dihydroxyvitamin D3 (BML-DM100-0001, Enzo Life Sciences), both reconstituted in $99.8 \%$ ethanol (Sigma-Aldrich), used at $10 \mathrm{nM}$ unless indicated, with identically diluted ethanol used as carrier control. IL-6 (BioLegend) and tocilizumab (a gift from C. Roberts in L. Taams' laboratory) were used in functional experiments.

Flow cytometry. Cells were stained $30 \mathrm{~min}$ at $4^{\circ} \mathrm{C}$ in a final volume of $100 \mu \mathrm{l}$ staining buffer with antibodies against CD4 (OKT4, Thermo Fisher Scientific), IL-6 (MQ2-13A5, BioLegend), LAG-3 (3DS223H, Thermo Fisher Scientific), CD49b (P1H5, Thermo Fisher Scientific) with 4,6-diamidino-2-phenylindole (DAPI) or LIVE/DEAD Fixable Aqua/Violet (Invitrogen) to exclude dead cells. For intracellular staining, cells were treated with the spiked addition of Phorbol 12-myristate 13-acetate (PMA) (50 $\mathrm{ng} \mathrm{ml}^{-1}$, Sigma-Aldrich), ionomycin (1 $\mu \mathrm{g} \mathrm{ml}^{-1}$, Sigma-Aldrich), GolgiStop (1×, BD Biosciences) and brefeldin A $(1 \times, \mathrm{BD}$ Biosciences) to medium then cultured $5 \mathrm{~h}$, subsequently washed in PBS, surface stained, treated with Cytofix/Cytoperm (eBioscience) and subsequent Perm/ Wash buffer (eBioscience) steps before incubation with intracellular antibodies for $30 \mathrm{~min}$ at $4^{\circ} \mathrm{C}$. Samples were washed and acquired on a LSRFortessa (BD Biosciences) or the Attune NxT Flow Cytometer (Invitrogen) within $24 \mathrm{~h}$ and analyzed using FlowJo v.9.9.6. Cell proliferation analysis was carried out by CFSE dilution in polyclonally activated $\mathrm{CD} 4^{+} \mathrm{CD} 25^{-} \mathrm{T}$ cells with carrier or VitD after $72 \mathrm{~h}$

Cell proliferation and activation markers were assessed in purified $\mathrm{CD} 4^{+}$cells with cellTrace Violet (CTV; C34556, Invitrogen) and surface staining against CD62L-FITC (MEL-14, BioLegend), CD69-PE-Cy7 (FN50, Invitrogen) or CD25-PE-Cy7 (BC96, Invitrogen) and LIVE/DEAD fixable near-IR (Invitrogen).

Cytokine measurement. Supernatants from 96-well plates were aliquoted and stored at $-20^{\circ} \mathrm{C}$. Cytokines were quantified by the Human/Mouse $\mathrm{T}_{\mathrm{H}} 1 / \mathrm{T}_{\mathrm{H}} 2 /$ $\mathrm{T}_{\mathrm{H}} 17$ Cytometric Bead Array (CBA) (BD Biosciences) using a FACSCanto II (BD Biosciences), analyzed by FCAP Array v.3.0 (BD Biosciences). Alternatively, we used the LEGENDplex Human Inflammation Panel 1 (13-plex) (740808,
BioLegend) with the Attune NxT Flow Cytometer. Cytokine assays were analyzed by FlowJo v.9.

For intracellular cytokine detection, the IFN- $\gamma / \mathrm{IL}-10$ Secretion Assay Detection kit (Miltenyi, 130-090-761) was used on live cells after $72 \mathrm{~h}$.

Immunoblotting. Cell extracts were prepared by lysis in RIPA (Thermo Fisher Scientific) including 1:250 diluted Protease Inhibitor Cocktail Set III (Calbiochem) and $5 \% 2$-mercaptoethanol, denatured at $95^{\circ} \mathrm{C}$ for $5 \mathrm{~min}$. Protein concentration was quantified using the Quick Start Bradford Protein Assay (Bio-Rad). Proteins resolved by SDS-PAGE on $10 \%$ Tris-glycine (Invitrogen) were transferred onto polyvinylidene fluoride membranes (Millipore) via a XCell II Blot Module (Thermo Fisher Scientific). Immunoblotting was performed with blocking in PBS $+10 \% \mathrm{w} / \mathrm{v}$ Blotting-Grade Blocker (1706404, Bio-Rad) for VDR, STAT3 and c-Jun or 3\% w/v BSA (Sigma-Aldrich) for pSTAT3 and phospho-c-Jun made up in PBS $+0.1 \% \mathrm{v} / \mathrm{v}$ Tween 20 (Sigma-Aldrich) then incubated overnight with antibodies against VDR (1:100 dilution, D-6, Santa Cruz), pSTAT3 (1:2,000 dilution, D3A7, Cell Signaling Technologies), STAT3 (1:1,000 dilution, 124H6, Cell Signaling Technologies), phospho-c-Jun (1:1,000 dilution, S63, R\&D Systems) or c-Jun (1:2,000 dilution, L70B11, Cell Signaling Technologies), followed by $2 \mathrm{~h}$ incubation with HRP-conjugated anti-mouse or anti-rabbit antibodies (1:1,000 dilution, TrueBlot, Rockland), then washed with PBS $+0.5 \% \mathrm{v} / \mathrm{v}$ Tween 20 . Targets were detected using ECL-Plus (Thermo Fisher Scientific), visualized by ImageQuant LAS 4000 mini (GE Healthcare) and quantified (Image Studio Lite, LI-COR).

Nuclear and cytoplasmic extracts were isolated (NE-PER Nuclear and Cytoplasmic Extraction, Thermo Fisher Scientific) and purity was determined by immunoblotting against HSP90 (1:2,000 dilution, C45G5, Cell Signaling Technologies) and H2A.X (1:1,000 dilution, D17A3, Cell Signaling Technologies).

For BACH2, snap-frozen cell pellets of $6 \times 10^{5}$ cells were resuspended directly in Laemmli $+5 \% 2$-mercaptoethanol (Bio-Rad) and denatured $\left(95^{\circ} \mathrm{C}\right.$ for $\left.5 \mathrm{~min}\right)$, cooled at room temperature, loaded and resolved at $120 \mathrm{~V}$ on a Criterion TGX Gel (Bio-Rad). Gel-bound proteins were transferred to nitrocellulose (Trans-Blot Turbo Midi, Bio-Rad) via a Trans-Blot Turbo (Bio-Rad). Membrane blocking occurred (PBS Odyssey Blocking Buffer, LI-COR) before addition of BACH2 (D3T3G, Cell Signaling Technologies) antibody in blocking buffer (1:5,000 dilution) then rotated overnight at $4{ }^{\circ} \mathrm{C}$. Membranes were washed three times (PBS $+0.1 \%$ Tween 20$)$ and exposed to IRDye 800CW goat anti-rabbit IgG secondary antibody (926-32211, LI-COR) in blocking buffer (1:5,000 dilution) for $1 \mathrm{~h}$ at room temperature. After three washes, antibody signals were visualized (Odyssey CLx, LI-COR). The membrane was re-probed with anti- $\beta$-tubulin diluted in blocking buffer ( 2146 , Cell Signaling Technologies, 1:1,000 dilution) and quantified (Image Studio v.5.2, LI-COR).

Human phospho-kinase antibody array. Proteome Profiler Human Phospho-Kinase Array (R\&D Systems) was carried out on VitD- or carrier-treated $\mathrm{CD} 4^{+}$cells. Cells were lysed in manufacturer's buffer, protein concentrations quantified by Quick Start Bradford Protein Assay (Bio-Rad) and samples adjusted to $800 \mu \mathrm{g} \mathrm{ml}^{-1}$ with lysis buffer. Then, $334 \mu \mathrm{l}$ was loaded per membrane and signals were amplified by Chemi-Reagent Mix imaged (ImageQuant LAS $4000 \mathrm{mini}$ ) and quantified (Image Studio Lite). Reference spots were used to normalize signals across membranes.

VDR and DAPI colocalization. VDR and DAPI colocalization was performed in $\mathrm{CD}^{+} \mathrm{T}$ cells stained with VDR antibody (1:250 dilution, D-6), anti-mouse-AlexaFluor647 (1:400 dilution, clone poly4053, BioLegend) and DAPI. Images were acquired via ImageStreamX running Inspire and VDR to DAPI colocalization assessed (IDEAS v.3.0, Amnis). A minimum of $1 \times 10^{5}$ events were acquired per sample.

Quantitative PCR. The $2-4 \times 10^{6}$ cells were lysed in $350 \mu$ Trizol (Life Technologies) and RNA extracted by Direct-zol Miniprep (Zymo Research) with on-column genomic DNA digest. RNA was quantified by spectrophotometry (NanoDrop) and reverse transcribed (qPCRBIO cDNA Synthesis, PCR Biosystems). qPCR in 384-well plates with SYBR Green PCR Master Mix (Life Technologies) was used with the ViiA7 real-time PCR system (Life Technologies). Reactions were carried out in triplicate with $18 s$ and UBC (geNorm 6 gene kit, ge-SY-6, PrimerDesign UK) as a reference alongside IL6 detection (Hs_IL6_1_SG QuantiTect Primer Assay, QIAGEN). TaqMan probes (Applied Biosystems) for CYP27B1 (Hs01096154_m1), VDR (Hs01045843_m1), IFNG (Hs00989291_m1), IL10 (Hs00961622_m1), CTSL (Hs00964650_m1), CYP27B1 (Hs01096154_m1) and 18S (Hs99999901_s1) were utilized on reverse-transcribed RNA (iScript, Bio-Rad) with TaqMan Master Mix (Thermo Fisher Scientific). The comparative Ct method was used for analysis using Viia7 software producing a Ct (relative quantity compared to a reference sample).

Inhibition and siRNAs. For STAT3 siRNA, memory $\mathrm{CD} 4^{+} \mathrm{T}$ cells were sorted and rested overnight in culture medium. Then, $5 \times 10^{6}$ cells were washed twice with PBS and nucleofected with 10 nM STAT3 Silencer Select siRNA (s745, sense GCACCUUCCUGCUAAGAUUTT and antisense 
AAUCUUAGCAGGAAGGUGCCT) or Silencer Select Negative Control (Thermo Fisher Scientific) using the Amaxa Human T Cell Nucleofector kit (Nucleofector 2b Device, U-014 program, Lonza). Nucleofected cells were cultured in medium for $6 \mathrm{~h}$ followed by dead cell removal (Lymphoprep, Axis-Shield). Then, $2 \times 10^{6}$ recovered cells were activated with anti-CD3/CD28 beads for $72 \mathrm{~h}$ and knockdown was assessed by immunoblot.

For chemical STAT3 inhibition, memory $\mathrm{CD}^{+} \mathrm{T}$ cells were sorted and rested overnight. Then, $2 \times 10^{6}$ cells were activated as before with VitD or carrier and additionally with $150 \mathrm{nM}$ curcubitacin I, Cucumis sativus L. (Calbiochem) or carrier (ethanol) for $72 \mathrm{~h}$

For VDR, CYP27B1 and CTSL siRNA, total $\mathrm{CD}^{+} \mathrm{T}$ cells were plate-activated with CD3/CD46 antibodies as described above. Dharmacon Accell SMARTPool siRNAs against CYP27B1 (E-009757-00-0005), VDR (E-003448-00-0005), CTSL (E-005841-00-0005), Non-targeting Control (D-001910-01-05) or Green Non-targeting Control (D-001950-01-05) were added to cultures at $1 \mu \mathrm{M}$ for $72 \mathrm{~h}$ with knockdown assessed by qPCR.

H3K27Ac and c-JUN CUT\&RUN. CUT\&RUN sequencing was performed on memory $\mathrm{CD}^{+} \mathrm{T}$ cells activated as above in the presence of carrier or VitD for $0.75 \mathrm{~h}, 48 \mathrm{~h}$ and $72 \mathrm{~h}$ using the published protocol of Skene et al..$^{55}$. Antibodies against H3K27Ac (ab4729, Abcam), c-JUN (60AB, Cell Signaling Technologies) and nonspecific IgG (31235, Thermo Fisher Scientific) were used with pAG-MNase (123461, Addgene) on $5 \times 10^{4}$ cells per target. CUT\&RUN (and CUT\&Tag below) buffer components were purchased from Sigma-Aldrich and Thermo Fisher Scientific. Post-CUT\&RUN, short DNA fragments were prepared for paired-end sequencing (NEB Ultra II, New England Biolabs) with NovaSeq (Illumina). Amplified libraries were quantified by high sensitivity fluorometry (DeNovix) and sized via the 4200 TapeStation (Agilent Technologies).

$\mathrm{H} 3 \mathrm{~K} 27 \mathrm{Ac}$ reads were aligned to the human reference genome (GRCh37; hg19) using Bowtie2 (ref. ${ }^{56}$ ) with parameters '-local -maxins 250' with sorting and indexing of aligned reads by SAMtools v.1.10 (ref. ${ }^{57}$ ); reads with mapping quality $<30$ were removed. H3K27Ac peaks were called by MACS2 (ref. ${ }^{58}$ ) with parameter '-f BAMPE -nomodel'. JUN reads were mapped without '-local' parameter. The $<120$-bp fragments were further sorted and indexed by SAMtools v.1.10 (ref. ${ }^{57}$ ). JUN-induced peaks were called by MACS2 parameters '-nomodel -g hs -f BAMPE -q 0.01 -SPMR -keep-dup all'. IgG BAM files were included as controls for MACS2. Peaks for H3K27Ac and JUN experiments were screened against previously characterized hypersequencable-regions (Hg19 Blacklist, CutRunTools $^{59}$ ). H3K27Ac and JUN mean binding intensities in peak regions were calculated by University of California Santa Cruz bigWigAverageOverBed. Peaks, combined from two conditions, with mean binding intensity $>0.2$ in at least one condition and FC of signal $>1.5$ were differential. Known motifs $\pm 100 \mathrm{bp}$ from differential peak summits were identified (HOMER, findMotifsGenome. $\mathrm{pl}$, parameter '-size given'). De novo motif footprinting for c-JUN was performed (CutRunTools ${ }^{74}$ ). Average cuts per bp proximal to JUN peaks was plotted as a histogram relative to IgG. CUT\&RUN tracks and heat maps were visualized using IGV (Broad Institute) and deepTools ${ }^{60}$, respectively.

De novo super-enhancer calling. SEs were called using ROSE (Rank Ordering of Super-Enhancers) ${ }^{37}$. H3K27Ac CUT\&RUN VitD-induced peaks were sorted/ indexed using SAMtools v.1.10 (ref. ${ }^{57}$ ) and executed with ROSE_main.py with carrier control. SE annotations were screened and corrected if needed. Hockey-stick plots of rank-ordered stitched-enhancers plotted against VitD minus carrier H3K27Ac signal were generated (RStudio v.1.2.5033)

VDR, STAT3 and BACH2 CUT\&Tag. VDR, STAT3 and BACH2 genome-wide binding in memory $\mathrm{CD} 4{ }^{+} \mathrm{T}$ cells activated in the presence of $10 \mathrm{nM}$ VitD or carrier for $96 \mathrm{~h}$ was detected by CUT\&Tag as per the published protocol (CUT\&Tag v.3, https://doi.org/10.17504/protocols.io.bcuhiwt6) ${ }^{61}$ using fixed nuclei ( 2 min fixation of isolated nuclei at room temperature) with 75,000 nuclei per target and antibodies targeting either VDR (D2K6W, Cell Signaling Technologies), STAT3 (D3Z2G, Cell Signaling Technologies), BACH2 (D3T3G) or nonspecific IgG (31235) diluted 1:50 accordingly and incubated with fixed nuclei for $1 \mathrm{~h}$ at room temperature. Secondary antibody (1:100 dilution) (ABIN101961, antibodies-online) was bound for $0.5 \mathrm{~h}$ at room temperature. Preloaded pA-Tn5 (C01070001, Diagenode) was tethered to antibodies for $1 \mathrm{~h}$ at room temperature. Libraries were sequenced as paired-ends by NovaSeq. Resulting reads were processed and mapped to $\mathrm{Hg} 19$ (CutRunTools), with peaks called (SEACR v.1.2, stringent, all mapped fragments at https://github.com/FredHutch/SEACR) and hypersequencable-regions subtracted. Known motif enrichment was determined $\pm 200 \mathrm{bp}$ from peak summits and data were visualized as in CUT\&RUN.

VDR ChIP-PCR. ChIP-PCR detection of VDR binding at the promoters of CYP24A1, STAT3 and IL6 was determined from $2 \times 10^{5} \mathrm{CD} 4^{+}$cells activated with VitD or carrier for $48 \mathrm{~h}$ followed by True MicroChIP (C01010132, Diagenode; three bioruptions per sample). Then, $4 \mu \mathrm{g}$ of anti-VDR (D-6) or IgG2a isotype control antibody (E5Y6Q, Cell Signaling Technologies) was used during overnight chromatin precipitation at $4^{\circ} \mathrm{C}$ before reverse-crosslinking and purification (MicroChIP DiaPure, Diagenode). qPCR primers were designed to amplify regions within $500 \mathrm{bp}$ upstream of the transcriptional start site for each respective gene: CYP24A1 (F: TGACCGGGGCTATGTTCG, R: GGCTTCGCA TGACTTCCTG); STAT3 (F: CTGTTCCGACAGTTCGGTGC, R: GCAGGACA TTCCGGTCATCTTC); and IL6 (F: GTAAAACTTCGTGCATGACTTCAGC, R: GGGGGAAAAGTGCAGCTTAGG). Triplicate qPCR using POWRUP SYBR Green (Thermo Fisher) was quantified via CFX384 (Bio-Rad) and results calculated as $\%$ Input $=2^{(-\Delta \mathrm{Ct}(\text { normalized ChIP) })}$.

RNA sequencing and analysis. Memory $\mathrm{CD} 4^{+} \mathrm{T}$ cells, including from $\mathrm{BACH} 2^{\mathrm{WT}}$ / ${ }_{\mathrm{L} 24 \mathrm{P}}$ patients, were activated with VitD or carrier. Then, $6 \times 10^{5}$ cells were pelleted at $300 \mathrm{~g}$ for $5 \mathrm{~min}$ and RNA was extracted by RNAqeous Micro (Thermo Fisher Scientific). RNA at $1 \mu \mathrm{g}$ total for each sample was subjected to NEBNext Poly(A) mRNA Magnetic Isolation (E7490) and resulting mRNA was prepared for RNA-seq by NEB Ultra II (New England Biolabs) and sequenced by HiSeq (Illumina).

The expression levels of all genes in RNA-seq libraries were quantified by 'rsem-calculate-expression' in RSEM v.1.3.1 (ref. ${ }^{62}$ ) with parameters '-bowtie-n 1 -bowtie-m 100 -seed-length 28 -bowtie-chunkmbs 1,000'. The bowtie index for RSEM alignment was generated by 'rsem-prepare-reference' on all RefSeq genes, downloaded from University of California Santa Cruz Table Browser in April 2017. EdgeR v.3.26.8 (ref. ${ }^{63}$ ) was used to normalize gene expression among all libraries and identify DEGs among samples. Microarray analysis sourced from GSE119416 was carried out using Partek Genomics Suite (Partek). DEGs were defined using the following criteria: at least $1.5 \times \mathrm{FC}$ in either direction at $P<0.05$ for microarray; at least $1.75 \times \mathrm{FC}$ in either direction at $\mathrm{FDR}<0.05$ for RNA-seq.

Single-cell RNA sequencing and analysis. COVID-19 bronchoalveolar lavage dataset. The pre-processed h5 matrix files for nine bronchoalveolar lavage (BAL) samples from patients with COVID-19 and four healthy control BAL samples were from GSE145926 (ref. ${ }^{64}$ ), GSE122960 (ref. ${ }^{65}$ ) and GSM3660650, respectively. Read mapping and basic filtering performed with Cell Ranger (10X Genomics). Further processing using Seurat v.3 (ref. ${ }^{66}$ ) was performed as follows: only genes found to be expressed in $>3$ cells and cells with gene number between 200 and 6,000 (unique molecular identifier (UMI) count $>1,000$ ) were retained. Cells with $>10 \%$ of their UMIs mapping to mitochondrial genes or cells with $<300$ features were discarded to eliminate low-quality cells. Filtering yielded 66,453 cells across 12 samples. Filtered count matrices were normalized by total UMI counts, multiplied by 10,000 and transformed to natural log space. Top 2,000 variable features determined by the variance stabilizing transformation function (FindVariableFeatures) using default parameters. Data were integrated using canonical correlation analysis (FindIntegrationAnchors/IntegrateData) with parameter k.filter $=140\left(\right.$ ref. $\left.{ }^{67}\right)$. Variants arising from library size and percentage of mitochondrial genes were regressed out (ScaleData, Seurat). Principal-component analysis (PCA) was performed and the top 50 principal components (PCs) were included in a UMAP. Clusters identified on a shared nearest neighbor (SNN) modularity graph using the top 50 PCs and the original Louvain algorithm. Cluster annotations are based on canonical marker genes.

Cells identified as ' $\mathrm{T}$ ' and 'cytotoxic T' were subsetted and re-processed. Samples C146 and C52 were removed due to low T cells (19 and 56 cells, respectively). Integration was performed using FindIntegrationAnchors/ IntegrateData with parameters: 'dims $=1: 30 \mathrm{k}$.filter $=124$ '. Normalization, variable feature detection, scaling, dimensionality reduction and clustering were performed using the top $30 \mathrm{PCs}$ and clustering resolution of 0.3 . Annotation was guided by marker genes (Extended Data Fig. 1c). The contaminating macrophage cluster annotated by canonical marker genes (for example CD14) was removed. Statistical differences were assessed by two-tailed Wilcoxon test. DEGs were defined by FC $>1.5$, adjusted $P$ value $<0.05$ and expression in at least $10 \%$ of cells in either cluster comparison.

Two clusters were annotated as $\mathrm{CD} 4^{+} \mathrm{T}$ cells, one with few cells but expressed similar markers to the main $\mathrm{CD} 4^{+}$cluster. The two were combined and considered together for analysis. DEGs between COVID-19 and healthy $\mathrm{CD}^{+} \mathrm{T}$ cells were visualized using Morpheus (https://software.broadinstitute.org/morpheus/). DEGs upregulated in COVID-19 CD4 ${ }^{+} \mathrm{T}$ cells were subjected to pathway analysis (Fig. 1c) using the Hallmark gene set collection from MSigDB (v.7.1) or drug prediction from the National Toxicology Program's DrugMatrix gene sets $(n=7,876)$ using EnrichR (Fig. 4f). Gene list scores were calculated by the AddModuleScore function in Seurat with a control gene set ( $n$ of 100 ). Correlations and ROC analyses on module scores comparing patients and healthy controls were performed in R or Prism v.8.4.0.

COVID-19 PBMC dataset. The pre-processed R objects for six PBMC samples from patients with COVID-19 and six healthy control PBMC samples were from GSE150728 (ref. ${ }^{68}$ ) with read mapping and filtering performed using Cell Ranger. The exon count matrices were further processed by Seurat as follows: only genes found to be expressed in more than ten cells were retained. Quality control steps for filtering the samples were performed as described previously ${ }^{68}$. Cells with $1,000-15,000$ UMIs and $<20 \%$ of reads from mitochondrial genes were retained. Cells with $>20 \%$ of reads mapped to RNA18S5 or RNA28S5 and/or expressed $>75$ genes per 100 UMIs were excluded. The SCTransform function was used to normalize the dataset and to identify variable genes ${ }^{68}$. PCA was performed and 
the top 50 PCs were included in UMAP dimensionality reduction. Clusters were identified on an SNN modularity graph using the top 50 PCs and the original Louvain algorithm. Cluster annotations were based on canonical marker genes. Gene list module scores were calculated using the AddModuleScore function in Seurat ${ }^{66}$. AUCs for module scores of every gene set in Hallmark and canonical pathways were curated by MSigDB ( $n=2,279$ gene sets $)^{69-71}$.

Gene set enrichment analysis and gene lists. GSEA was carried out as published ${ }^{69}$ $\operatorname{Tr} 1$ gene lists were obtained from GSE139990. Data were filtered to include only genes expressed at $>0.25$ counts per million in at least two samples. Trimmed mean of $\mathrm{M}$ values (TMM) were normalized within edgeR v.3.28.1 and differential expression was performed using glmQLFit and glmQLFTest edgeR functions. $\operatorname{Tr} 1$ signatures were defined as DEGs (at least fourfold change in either direction at FDR $<0.05)$ between $\operatorname{Tr} 1$ and $\mathrm{T}_{\mathrm{H}} 0$ cells. $\mathrm{T}_{\mathrm{H}} 1 / \mathrm{T}_{\mathrm{H}} 2 / \mathrm{T}_{\mathrm{H}} 17$ gene lists were obtained from transcriptomes of sorted mouse $\mathrm{CD} 4^{+} \mathrm{T}$ cell subsets ${ }^{72}$. VitD-regulated genes were defined as described in RNA-seq methods. BACH2-bound and regulated genes were obtained from GSE45975 (ref. ${ }^{38}$ ). All gene lists used in this manuscript are in Supplementary Table 8.

Psoriasis lesional skin staining, imaging and analysis. Informed consent for all patients with psoriasis and data were obtained under the Psoriasis, Atherosclerosis and Cardiometabolic Disease Initiative (PACI, 13-H-0065). Samples included three patients with psoriasis not on VitD and two patients with psoriasis on VitD supplementation. Three-millimeter punch-biopsies were collected from psoriasis lesional skin and placed into $10 \%$ formalin. Seven-micrometer skin sections were mounted on glass slides, de-waxed and antigens were retrieved via the citrate buffer method. Sections were washed once in PBS and blocked for $20 \mathrm{~min}$ at room temperature in 10\% normal goat serum then stained with anti-CD3 (1:50 dilution, F7.2.38, Thermo Fisher Scientific) and anti-BACH2 (1:40 dilution, D3TG) primary antibodies overnight at $4{ }^{\circ} \mathrm{C}$. The next day sections were washed in $0.01 \mathrm{M}$ PBS and Alexa Fluor 488 and Alexa Fluor 594 (1:100 dilution, Jackson Immuno Research Laboratories, 115-546-062 and 111-586-047), secondary antibodies were added for $1 \mathrm{~h}$ at room temperature and then sections were washed in $0.01 \mathrm{M}$ PBS. Hoechst was added to the samples for $10 \mathrm{~min}$, then samples were washed and cover-slipped with Fluoromount G. Images were collected on a Zeiss 780 inverted confocal microscope at $\times 40$ with oil immersion and analyzed (ImageJ). Representative images were prepared in Zen Blue v.3.1 (Zeiss). For each sample, 5-6 images were acquired. To quantify the frequency of immune cells in the dermis, images were cropped to $1,200 \times 1,200$ pixels to exclude the epidermis and nuclei numbers in the $408 \mathrm{~nm}$ channel were determined. The number of BACH2-positive cells in the $594 \mathrm{~nm}$ channel were calculated per image and the number of $\mathrm{BACH} 2$ foci per $\mathrm{BACH} 2$-positive cell were recorded.

Data presentation and statistical analysis. Figures were prepared using Adobe Illustrator (Adobe). Statistical analysis and graphical visualizations were carried out in GraphPad Prism (v.8.4.0), XLstat Biomed (v.2017.4), DataGraph v.4.5.1 (Visual Data Tools), Cytoscape 3 (ref. ${ }^{73}$ ) and Circos Table Viewer v.90.63.9 (ref. ${ }^{74}$ ). Statistical analyses were performed using appropriate paired or unpaired parametric and nonparametric tests, as required. Multiple comparisons were performed using ANOVA. $P$ values $<0.05$ were considered statistically significant throughout.

Reporting Summary. Further information on research design is available in the Nature Research Reporting Summary linked to this article.

\section{Data availability}

Data generated for this study are deposited at the Gene Expression Omnibus under GSE154741. Source data are provided with this paper.

\section{References}

55. Skene, P. J., Henikoff, J. G. \& Henikoff, S. Targeted in situ genome-wide profiling with high efficiency for low cell numbers. Nat. Protoc. 13, 1006-1019 (2018).

56. Langmead, B. \& Salzberg, S. L. Fast gapped-read alignment with Bowtie 2. Nat. Methods 9, 357-359 (2012).

57. Li, H. et al. The Sequence Alignment/Map format and SAMtools. Bioinformatics 25, 2078-2079 (2009).

58. Zhang, Y. et al. Model-based analysis of ChIP-seq (MACS). Genome Biol. 9 , R137 (2008)

59. Zhu, Q., Liu, N., Orkin, S. H. \& Yuan, G.-C. CUT\&RUNTools: a flexible pipeline for CUT\&RUN processing and footprint analysis. Genome Biol. 20, 192 (2019).

60. Ramírez, F. et al. deepTools2: a next generation web server for deep-sequencing data analysis. Nucleic Acids Res. 44, W160-W165 (2016).

61. Kaya-Okur, H. S. et al. CUT\&Tag for efficient epigenomic profiling of small samples and single cells. Nat. Commun. 10, 1930 (2019).

62. Li, B. \& Dewey, C. N. RSEM: accurate transcript quantification from RNA-seq data with or without a reference genome. BMC Bioinf. 12, 323 (2011).
63. Robinson, M. D., McCarthy, D. J. \& Smyth, G. K. edgeR: a Bioconductor package for differential expression analysis of digital gene expression data. Bioinformatics 26, 139-140 (2010).

64. Liao, M. et al. Single-cell landscape of bronchoalveolar immune cells in patients with COVID-19. Nat. Med. 26, 842-844 (2020).

65. Reyfman, P. A. et al. Single-cell transcriptomic analysis of human lung provides insights into the pathobiology of pulmonary fibrosis. Am. J. Resp. Crit. Care 199, 1517-1536 (2019).

66. Tirosh, I. et al. Dissecting the multicellular ecosystem of metastatic melanoma by single-cell RNA-seq. Science 352, 189-196 (2016)

67. Stuart, T. et al. Comprehensive Integration of single-cell data. Cell 177, 1888-1902 (2019).

68. Wilk, A. J. et al. A single-cell atlas of the peripheral immune response in patients with severe COVID-19. Nat. Med. 26, 1070-1076 (2020)

69. Subramanian, A. et al. Gene set enrichment analysis: a knowledge-based approach for interpreting genome-wide expression profiles. Proc. Natl Acad. Sci. USA 102, 15545-15550 (2005).

70. Liberzon, A. et al. Molecular signatures database (MSigDB) 3.0. Bioinformatics 27, 1739-1740 (2011).

71. Liberzon, A. et al. The molecular signatures database hallmark gene set collection. Cell Syst. 1, 417-425 (2015).

72. Stubbington, M. J. et al. An atlas of mouse $\mathrm{CD} 4^{+} \mathrm{T}$ cell transcriptomes. Biol. Direct 10, 14 (2015).

73. Shannon, P. et al. Cytoscape: a software environment for integrated models of biomolecular interaction networks. Genome Res. 13, 2498-2504 (2003).

74. Krzywinski, M. et al. Circos: an information aesthetic for comparative genomics. Genome Res. 19, 1639-1645 (2009).

\section{Acknowledgements}

The authors thank patients who contributed samples for this study. We acknowledge G. Denisov and the NIH HPC (Biowulf) for their efforts in maintaining essential bioinformatic programs. We thank Q. Zhu, N. Liu, D. Janssens and S. Henikoff for critical discussions related to CUT\&RUN experimentation and data analysis. This work was supported by the Wellcome Trust (grant 097261/Z/11/Z to B.A.), the Crohn's and Colitis Foundation of America (grant CCFA no. 3765 - CCFA genetics initiative to A.L.), British Heart Foundation (grant RG/13/12/30395 to G.L.), the National Institute of General Medical Sciences (R35GM138283 to M.K.), the Showalter Trust (research award to M.K.), German Research Foundation (DFG scholarship to T.F.; FR 3851/2-1), the NIDDK (DK12262401A1 to D.P.) and the National Agency of Research and Development of Chile (grant PAI79170073 to E.N.L.). Research was also supported by the National Institute for Health Research (NIHR) Biomedical Research Centre based at Guy's and St Thomas' NHS Foundation Trust and King's College London and/or the NIHR Clinical Research Facility. The views expressed are those of the authors and not necessarily those of the NHS, the NIHR or the Department of Health. This research was supported (in part) by the Intramural Research Programs of the NIDDK (project no. ZIA/DK075149 to B.A), the National Heart, Lung and Blood Institute (project nos. ZIA/Hl006223 to C.K. and ZIA/HL006193 to N.M.), the NIAID (project no. ZIA/AI001175 to M.S.L.) of the NIH. D.C. is supported by an NIH Office of Dietary Supplements research scholar award.

\section{Author contributions}

Single-cell data analysis was performed by T.F., B.Y., Z.Z. and J.B. Bulk RNA experiments and analyses were carried out by T.F, D.C., L.W. and N.C. CUT \& RUN and analysis was carried out by D.C., L.W., P.L. and A.K. CUT \& Tag and analysis was carried out by D.C. Other wet-laboratory experiments were conducted by R.M., T.F., E.N.-L., H.T., E.E.W. C.K., D.K., B.A. and S.J. Patient samples were provided by A.F.F., A.M., D.M.S., N.M., M.J.R.-B., K.N.V., D.S.C. and N.C., who also analyzed data. D.P., S.J., P.L., M.S.L., N.M., C.K., N.C., G.L. and A.L. provided intellectual input, interpreted data, provided supervision of wet-laboratory work and helped write the paper. R.M., T.F., D.C., M.S.L., A.L., M.K. and B.A. wrote the manuscript. M.K. and B.A. conceived and supervised the project.

\section{Competing interests}

The authors declare no competing interests.

\section{Additional information}

Extended data is available for this paper at https://doi.org/10.1038/s41590-021-01080-3. Supplementary information The online version contains supplementary material available at https://doi.org/10.1038/s41590-021-01080-3.

Correspondence and requests for materials should be addressed to Majid Kazemian or Behdad Afzali.

Peer review information Nature Immunology thanks Kazuhiko Igarashi and the other, anonymous, reviewer(s) for their contribution to the peer review of this work. Zoltan Fehervari was the primary editor on this article and managed its editorial process and peer review in collaboration with the rest of the editorial team.

Reprints and permissions information is available at www.nature.com/reprints. 
a

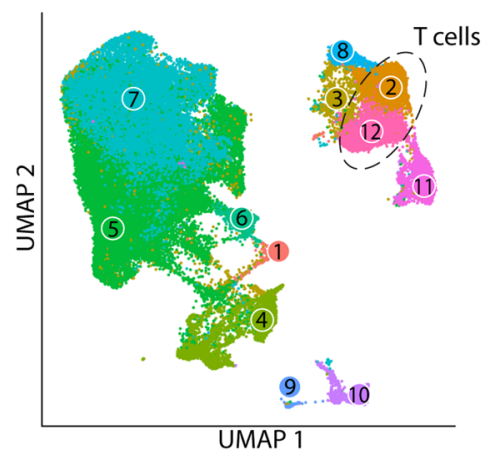

(1) B cells
(2) T cells (cytotoxic)
(3) Doublets
(4) Epithelial
(5) Macrophages
(6) $\mathrm{mDC}$
(7) Monocytes
(8) NK cells
(9) pDC
(1) Plasma cells
(11) Mixed proliferating lymphocytes
(12) T cells

b

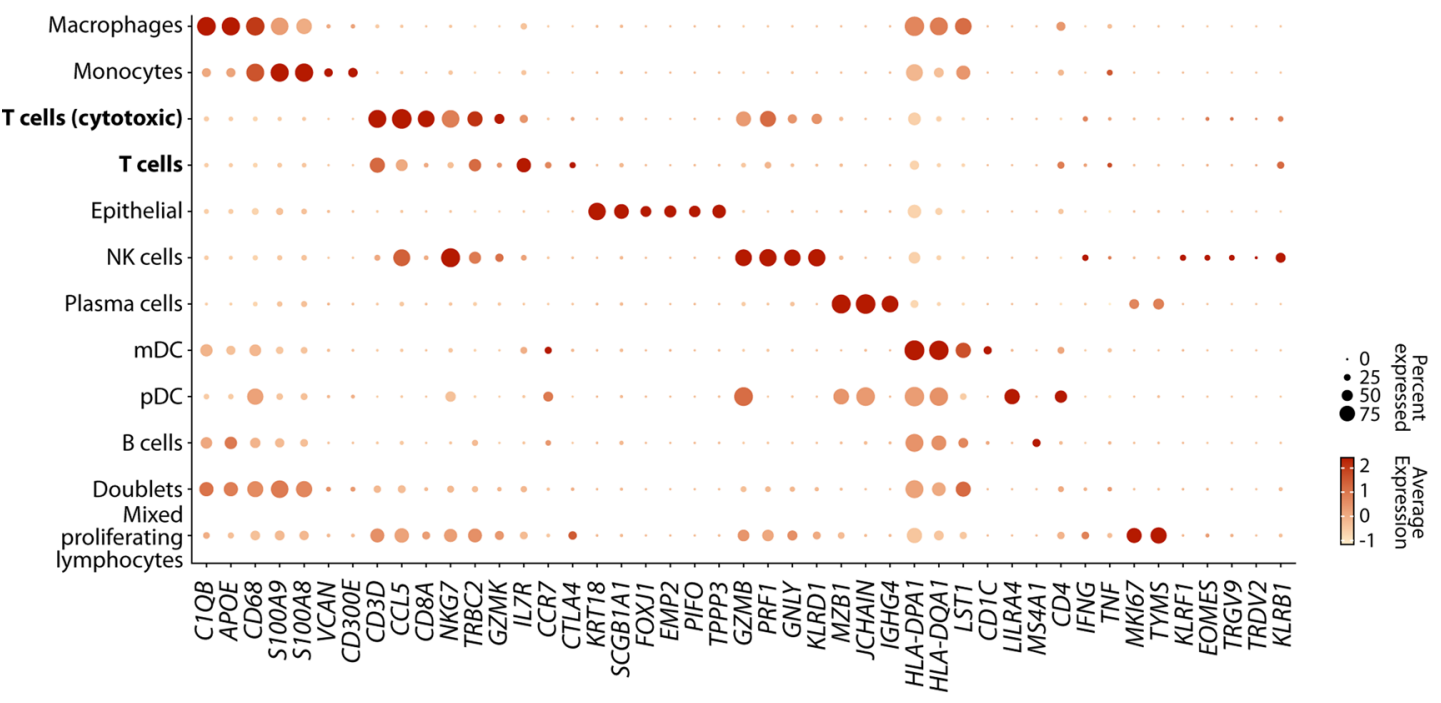

c

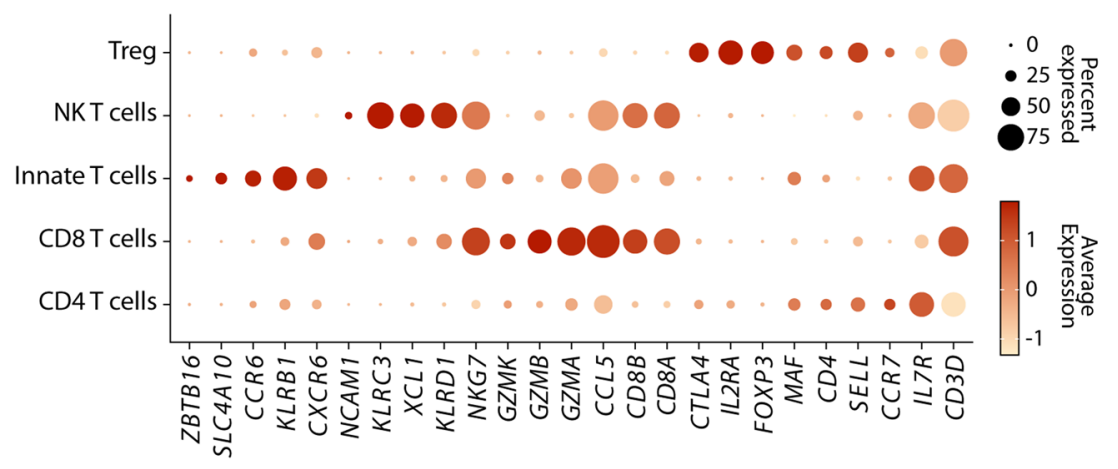

e

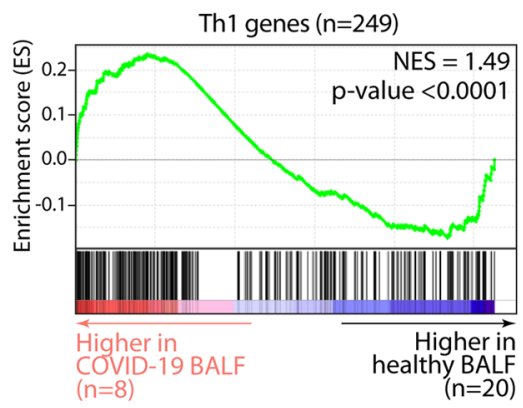

d

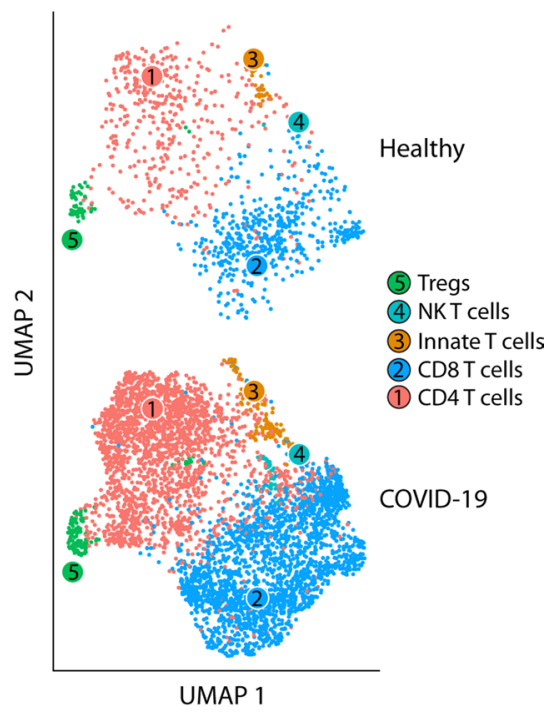

Extended Data Fig. 1 | See next page for caption. 


\section{NATURE IMMUNOLOGY}

Extended Data Fig. 1 | Cellular phenotypes of CD4 ${ }^{+}$T cells in BALF of patients with COVID-19. a-b, UMAP representation of scRNAseq showing main clusters of cells from bronchoalveolar lavage fluid (BALF) of patients with COVID-19 and healthy controls (a) and dot plot depicting expression of select marker genes for each cluster (b). Highlighted in both $\mathbf{a}$ and $\mathbf{b}$ are clusters 2 and 12, which represent T lymphocytes. c, Dot plot showing expression of select marker genes for clusters of cells depicted in Fig. 1a. d, UMAP projection of scRNAseq showing sub-clustering of T cells from bronchoalveolar lavage fluid (BALF) of healthy controls (above) and patients with COVID-19 (below). e, GSEA showing genes more highly expressed in bulk RNA-seq of BALF cells obtained from patients $(n=8)$ with COVID-19 compared to healthy controls $(n=20)$ are enriched in Th1 genes. Box and whisker plots (right) shows the expression of $1 L 10$ mRNA in these samples, indicating the median and extending to the minimum, maximum, 25\% and $75 \%$ quartiles. Data in a-b are from $n=9$ patients with COVID-19 and $n=4$ healthy controls, sourced from GSE145926 and GSE122960. Data in c-d are from the same sources but with $n=8$ patients with COVID-19 and $n=3$ healthy controls (one sample from each was removed due to too low CD4 ${ }^{+}$T cell numbers). Data in e are from $n=8$ patients with COVID-19 and $n=20$ healthy subjects, obtained from HRA000143. ${ }^{\star \star \star \star} p<0.0001$ by two-sided Mann-Whitney U-test. 
a

b
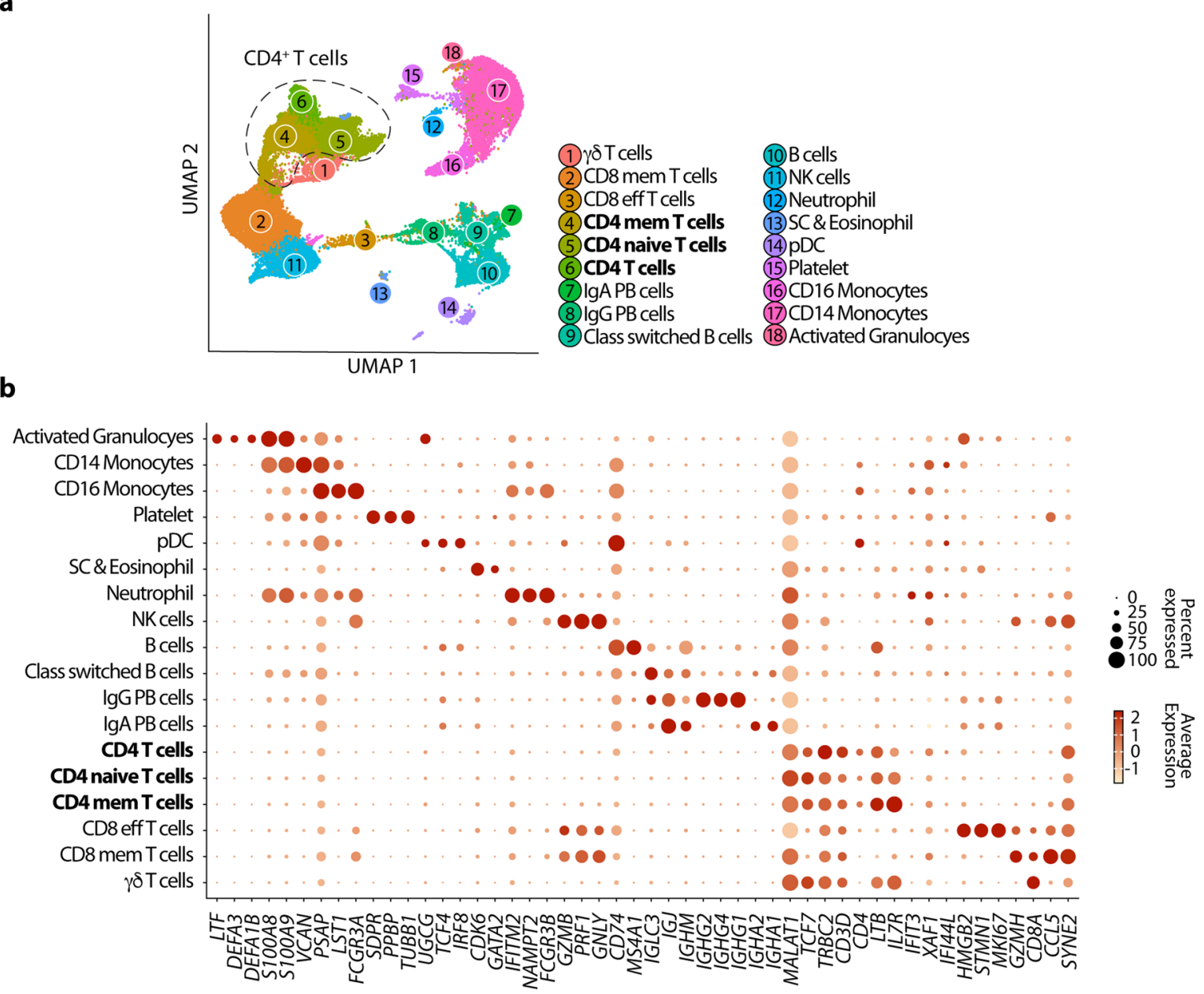

C

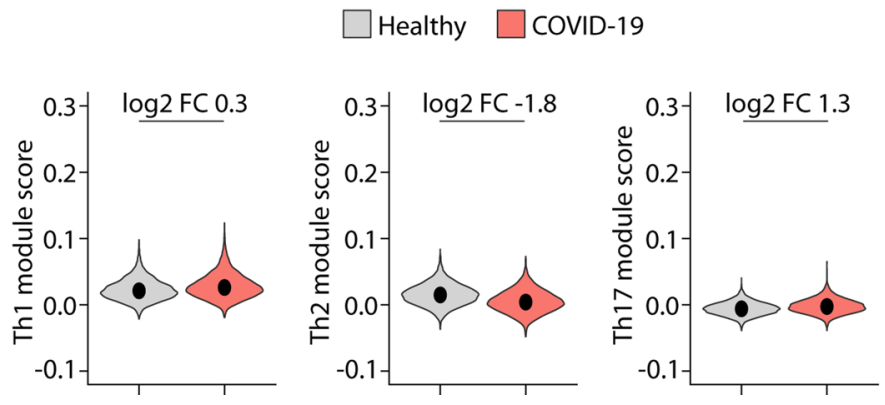

Extended Data Fig. 2 | Circulating CD4+ $\mathbf{T}$ cells of patients with COVID-19 are not Th1 biased. a-b, UMAP representation of scRNAseq showing main clusters of cells from peripheral blood mononuclear cells (PBMC) of patients with COVID-19 and healthy controls (a) and dot plot depicting expression of select marker genes for each cluster (b). Highlighted in both $\mathbf{a}$ and $\mathbf{b}$ are clusters 4,5 and 6 , which represent CD4+ T lymphocytes. c, Violin plots showing expressions of Th1, Th2 and Th17 genes, respectively, summarized as module scores, in PBMC CD4+ ${ }^{+}$cells of patients with COVID-19 and healthy controls. Data in a-c are from $n=6$ patients with COVID-19 and $n=6$ healthy subjects, obtained from GSE150728. 
a

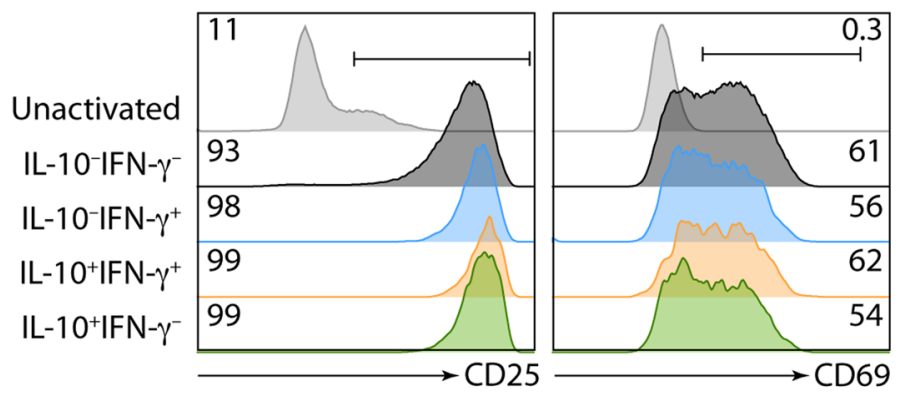

C

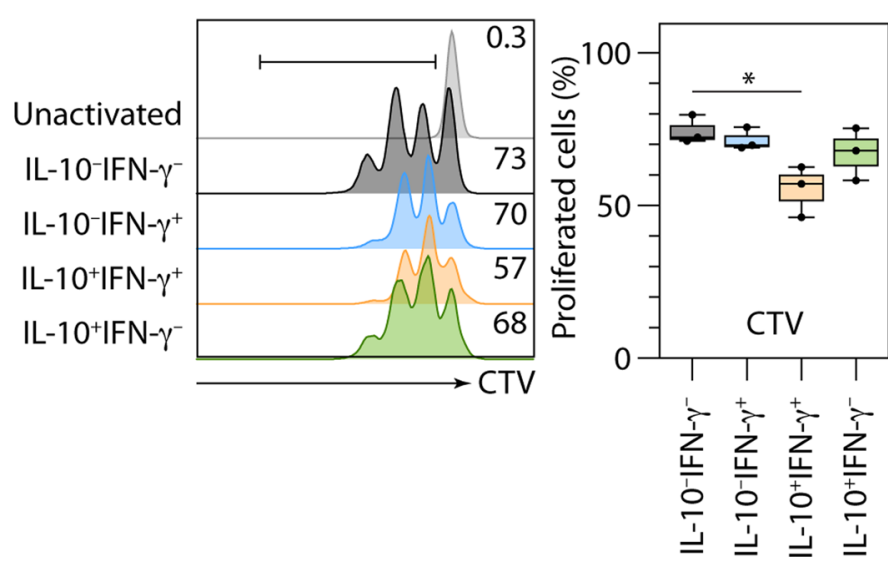

b

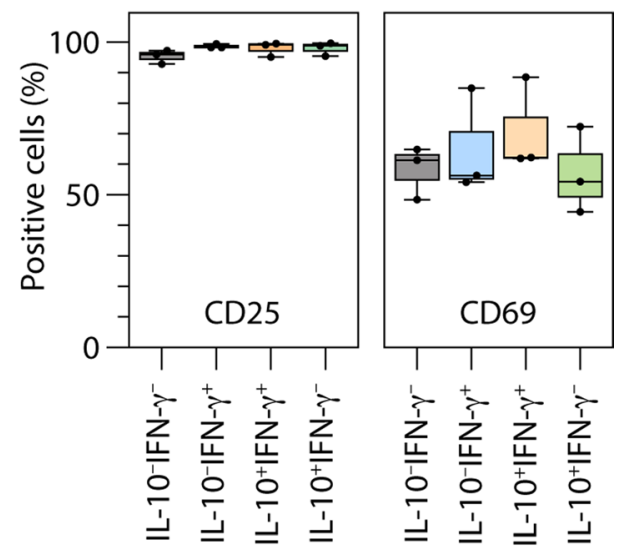

Extended Data Fig. 3 | Expression of activation markers and proliferation of anti-CD3 + anti-CD46-activated cells. CD4 ${ }^{+} T$ cells were activated as before using anti-CD3 + anti-CD46 in culture plates. After $48 \mathrm{~h}$ cells were stained for IFN- $\gamma$ and IL-10 and co-stained with CD25 or CD69. Separately, CTV-labeled CD4+ T cells were activated in the same manner. $\mathbf{a}-\mathbf{b}$, representative flow cytometry histograms (a) and cumulative data from $n=3$ independent experiments (b) of CD25 and CD69 expression in cells at each stage of cytokine secretion. $\mathbf{c}-\mathbf{d}$, representative CTV dilution representing cells that had undergone proliferation (c) and cumulative data from $n=3$ independent experiments (d). Box and whisker plots in $\mathbf{b}$ and $\mathbf{d}$ show the medians and extend to the minimum, maximum, $25 \%$ and $75 \%$ quartiles. ${ }^{\star} p<0.05$ by two-sided ANOVA. All other comparisons were non-significant. 


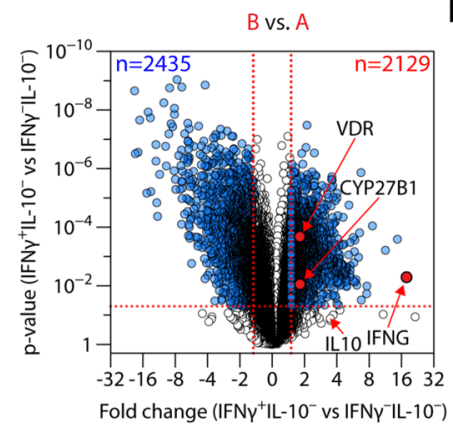

b

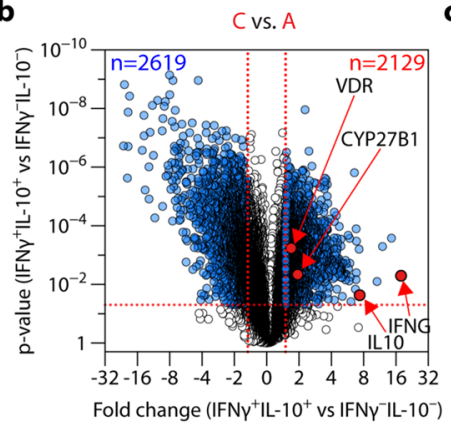

$c$

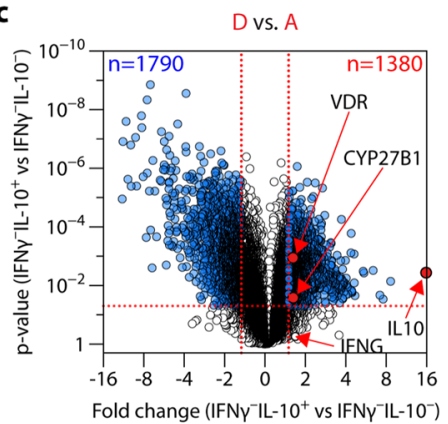

d

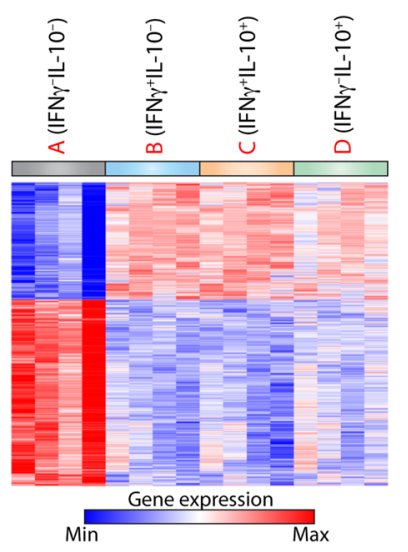

f

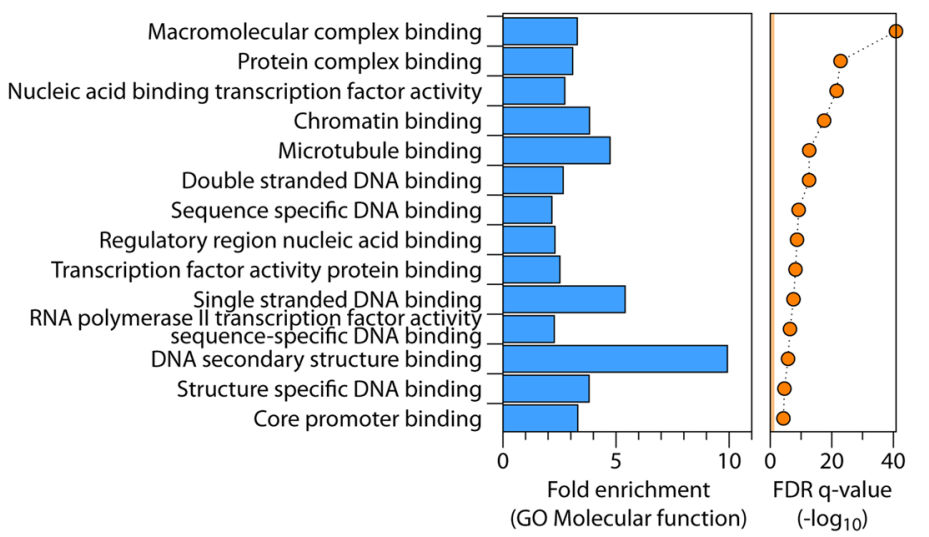

e

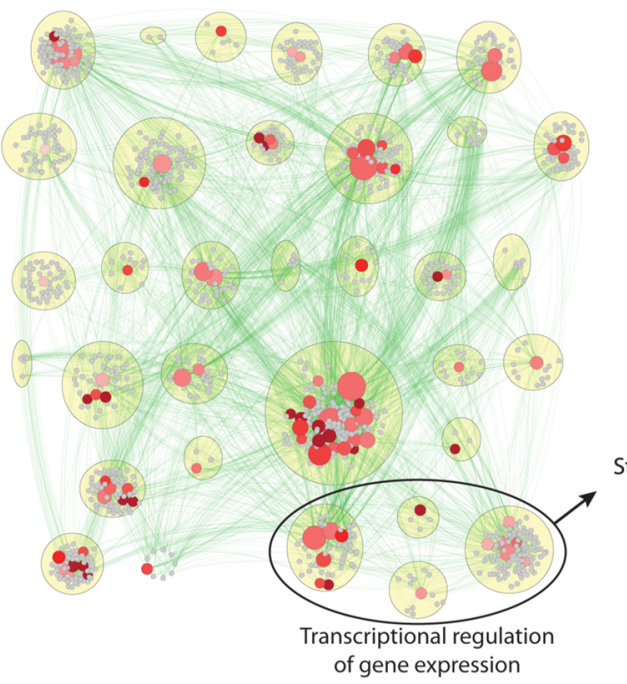

Red-filled nodes: pathways Gray nodes: genes

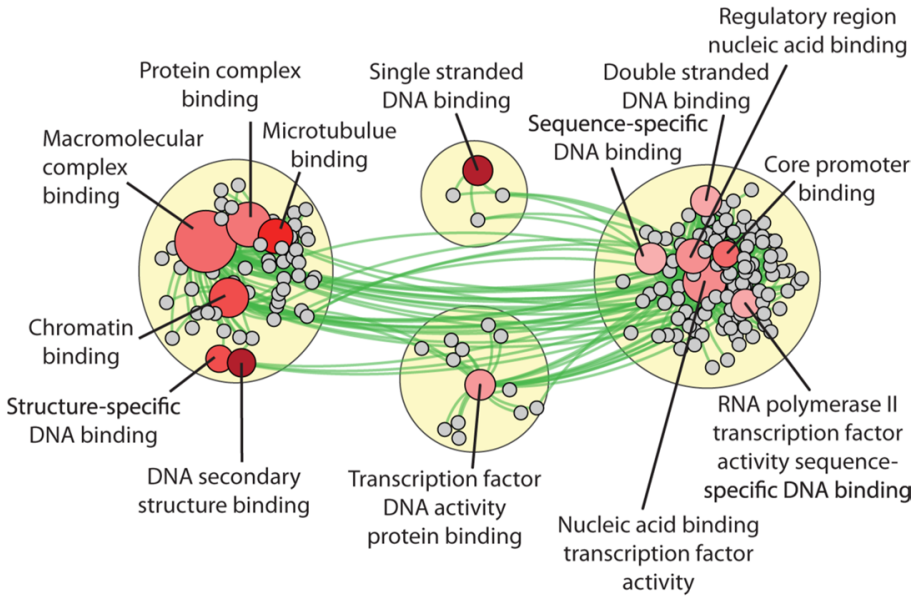

Pathway node fill: $1 \longdiv { 5 }$
Pathway node size: $40 \bigcirc 53$ $-\log 10$ FDR

Extended Data Fig. 4 | Complement-activated CD4 ${ }^{+}$T cells are enriched in transcription factors. a-c, Volcano plots showing differentially expressed genes (DEGs) following activation of CD4+ $4^{+}$cells with $\alpha-C D 3+\alpha-C D 46$, comparing IFN- $\gamma^{+}$IL-10- cells (a), IFN- $\gamma^{+} I L-10^{+}$cells $(\mathbf{b})$ and IFN- $\gamma^{-I L-10^{+}}$cells (c) to IFN- $\gamma^{-I L 10^{-}}$cells, respectively. DEGs are defined as at least 1.5-fold change in either direction at unadjusted p-value $<0.05$ using two-sided ANOVA. Marked in a-c are the IFNG, IL10, VDR and CYP27B1 genes. d, Heatmap showing expression of the 2023 shared DEGs in a-c. e, ClueGo analysis for molecular function terms in the 2023 DEGs shown in $\mathbf{d}$ represented as a Cytoscape visualization. Genes are shown in gray, enriched molecular function terms are in red scaled to reflect fold enrichment and edges link genes to molecular function terms. Node sizes reflect enrichment significance. Related terms grouped as families within yellow circles. Four such families represent transcriptional regulation of gene expression and are shown in the inset on the right. $\mathbf{f}$, The top 14 transcription factor molecular function terms are shown, with associated fold enrichments and FDR q-values. Data in a-f are from $n=4$ experiments. 


\section{$\begin{array}{lll}\text { CTSL } & \text { CYP27B1 } & \text { VDR }\end{array}$}
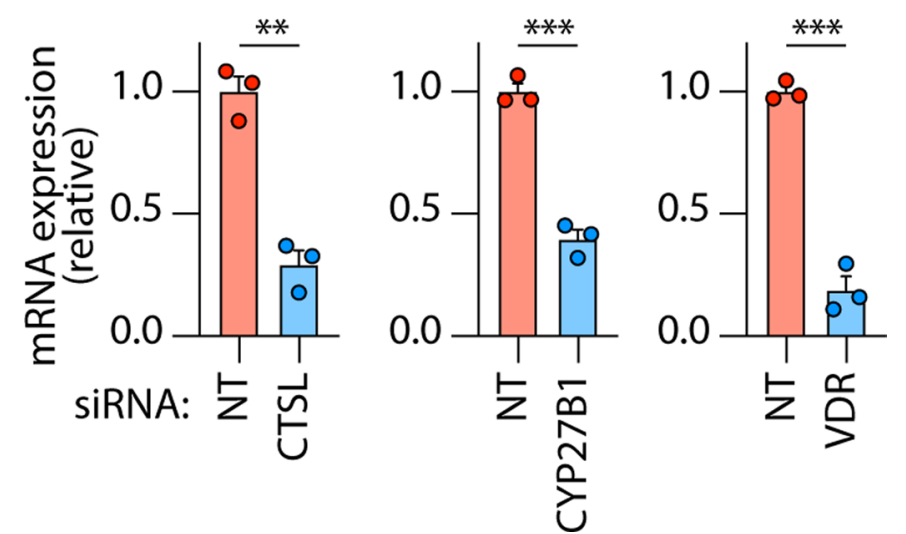

Extended Data Fig. 5 | Efficiency of silencing of indicated targets by siRNA from Fig. 2j. Shown are cumulative data from $n=3$ independent experiments. Bars show mean + sem. NT, non-targeting. ${ }^{\star \star} p<0.01^{\star \star \star} p<0.001$ by unpaired two-sided t-test. 
a

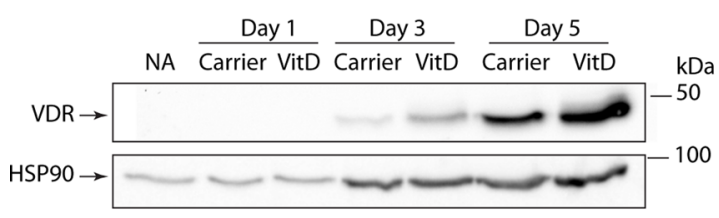

C
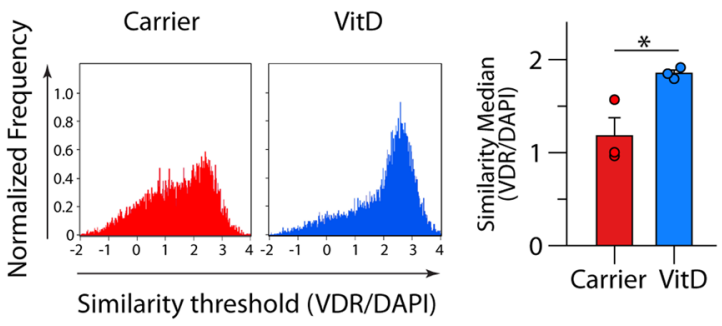

e

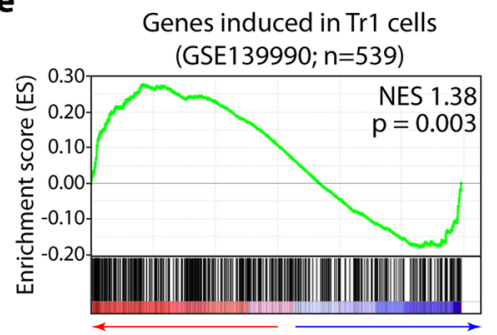

Higher in Carrier Higher in VitD

\section{f}

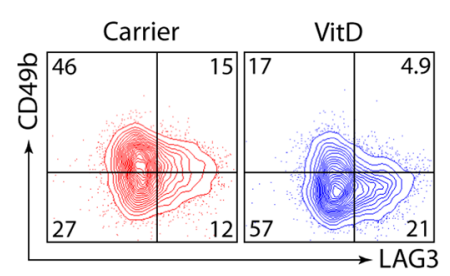

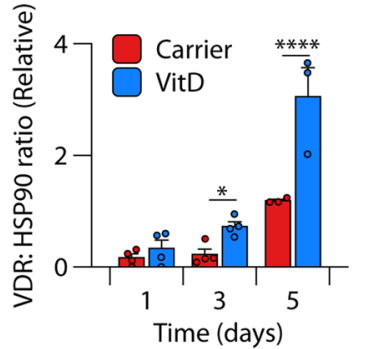

b

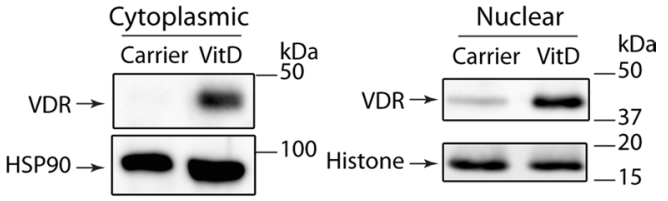

d

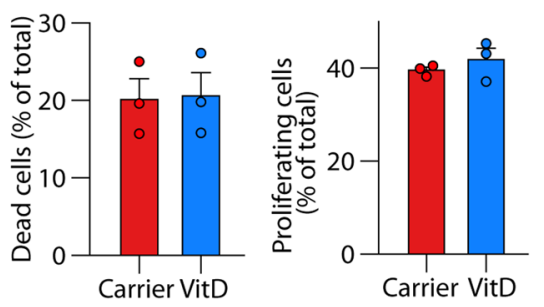

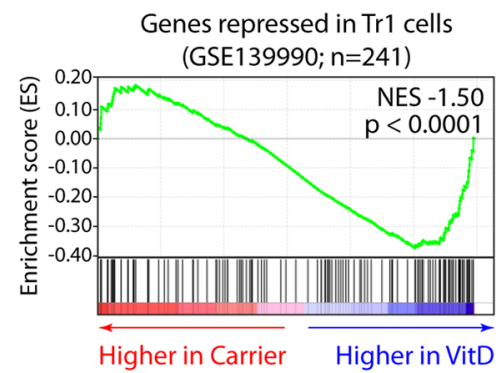
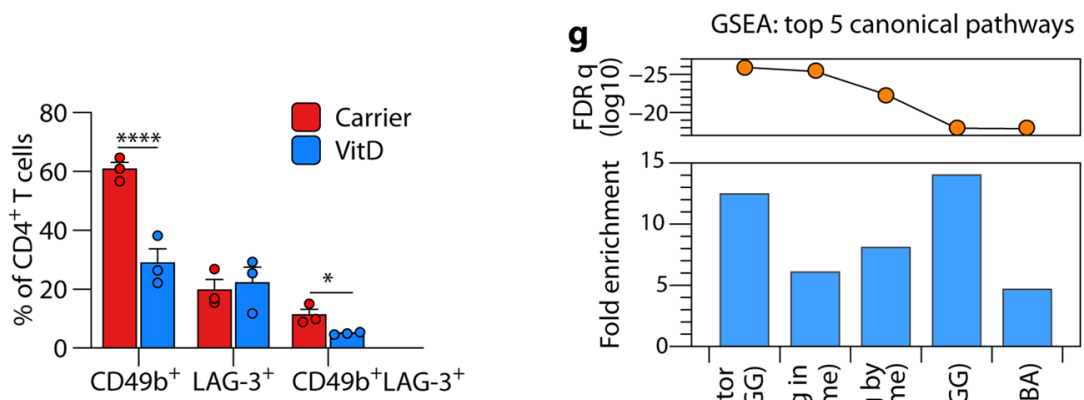

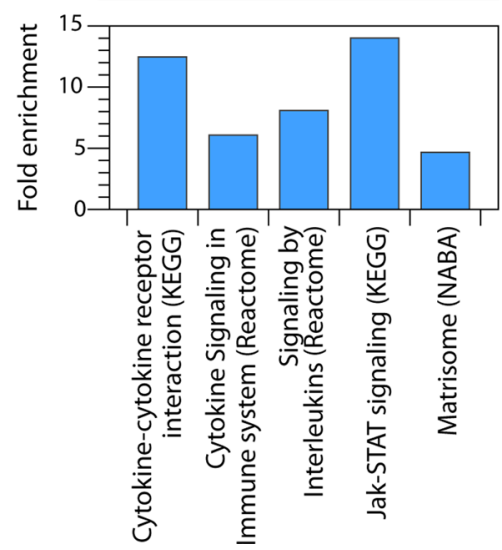

Extended Data Fig. 6 | Phenotype of Vitamin D treated cells. a, Western blot (left) and cumulative data (right) for VDR at days 1, day 3 and day 5, with Hsp90 as loading control, in both carrier and VitD treated CD4+ $\mathrm{T}$ cells. b. Representative immunoblots for VDR and indicated housekeeping proteins in nuclear and cytoplasmic extracts of carrier and VitD treated CD4 ${ }^{+} T$ cells. c, Co-localization of VDR and DAPI in carrier and VitD treated CD4 ${ }^{+}$ $T$ cells, measured on day 2 using ImageStream. Shown are representative frequency histograms indicating overlap between VDR and DAPI in the entire population (left) and cumulative data from $n=3$ independent experiments (right). d, Cell death assessed by live/dead stain and proliferation assessed by CFSE dilution in CD4 ${ }^{+} T$ cells treated with carrier or VitD after 3 days of culture. e, GSEA showing genes more highly expressed in CD4 ${ }^{+} T$ cells treated with carrier compared to VitD are enriched in Tr1-induced genes (left panel) and genes more highly expressed in CD4 ${ }^{+} \mathrm{T}$ cells treated with VitD compared to carrier are enriched in Tr1-repressed genes (right panel; curated from GSE139990). f, Representative flow cytometry plot showing CD49b and LAG-3 expression in CD4 ${ }^{+}$T cells, with carrier and VitD treatment (left) and quantification of cumulative data (right). $\mathbf{g}$, Top 5 MSigDB canonical pathways enriched in DEGs of VitD vs carrier treated CD4 ${ }^{+} T$ cells (see Fig. 3a). Unless indicated, all cells in Supplementary Fig. 6 have been activated with $\alpha-C D 3+\alpha-C D 28$. Bars represent mean + sem throughout. All experiments have been carried out $n=3$ times. Shown in e are unadjusted empirical p-values; NES $=$ normalized enrichment score. ${ }^{\star} \mathrm{p}<0.05,{ }^{\star \star \star \star} \mathrm{p}<0.0001$ by 2 -way ANOVA $(\mathbf{a}, \mathbf{f})$ and paired t-test (c). All statistical analyses are two-sided. 
a

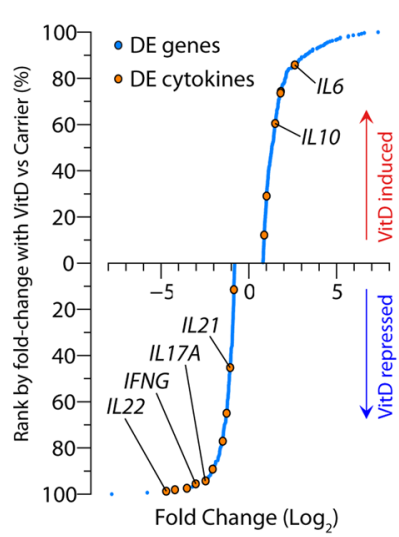

e
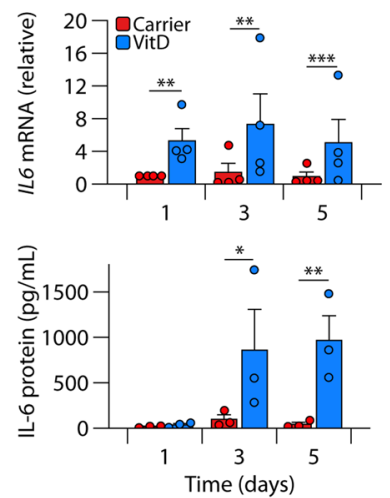

g

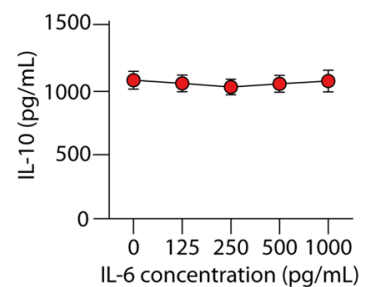

i

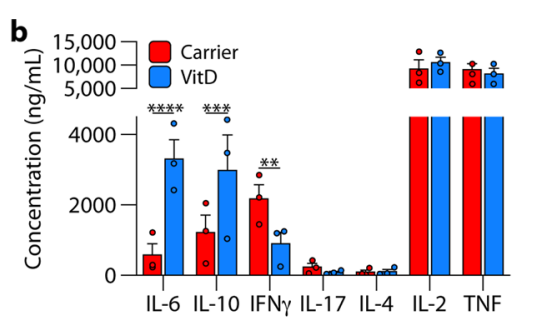

c

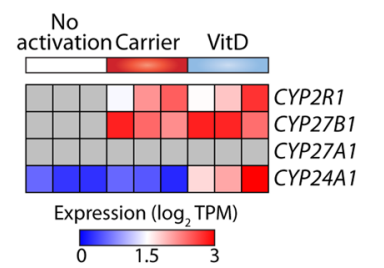

f

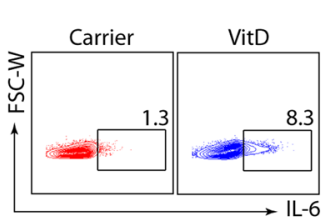

h

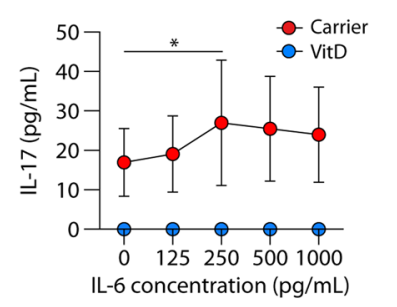

j

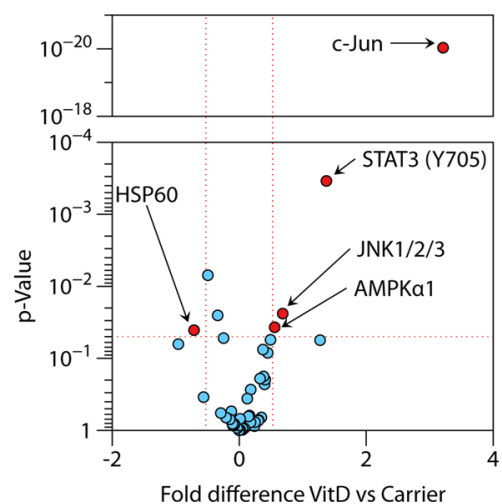

d
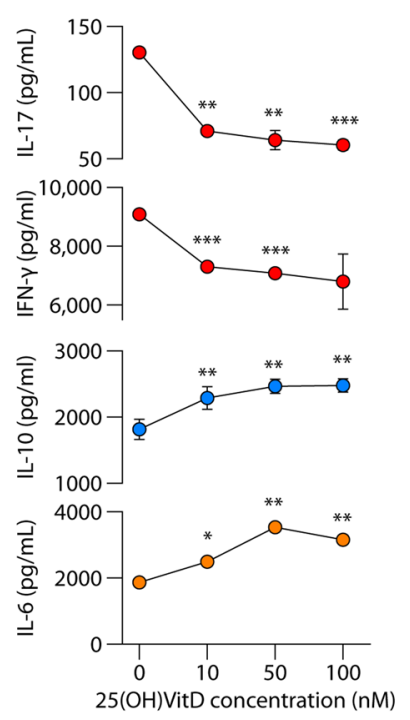

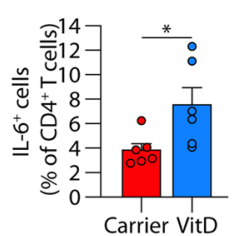

k

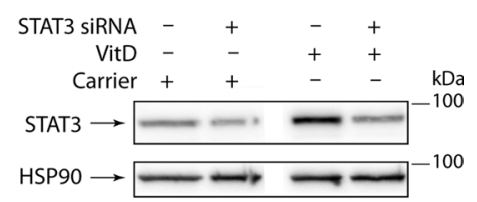

I

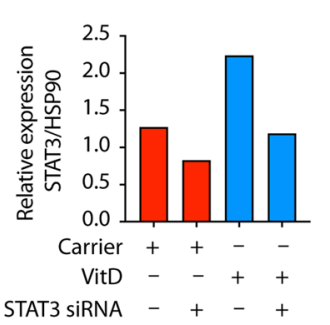

\section{Extended Data Fig. 7 | See next page for caption.}


Extended Data Fig. 7 | Hierarchy of cytokines induced by Vitamin D and modulation of IL-6 effects and the phosphor-kinase proteome by Vitamin D. a, Differentially expressed genes (DEGs) between VitD and carrier treated CD4+ T cells (see Fig. 3a,b) ranked by fold change. Each DEG is marked by a blue dot; each differentially expressed cytokine is marked by an orange dot. Select cytokines have been labelled. $\mathbf{b}$, Cytokine concentrations in supernatants of $\mathrm{CD} 44^{+} \mathrm{T}$ cell cultures after 5 days of treatment with carrier or VitD. c, Heatmap showing mRNA expressions (log2 TPM) of the 25-hydroxylase enzymes (CYP2R1 and CYP27A1), the 1 $\alpha$-hydroxylase enzyme (CYP27B1) and the 24-hydroxylase enzyme (CYP24A1), responsible for the two steps of Vitamin D activation and its subsequent inactivation, respectively. Data are from CD4 ${ }^{+} T$ cells activated with $\alpha-C D 3+\alpha-C D 28$ and cultured with either carrier or VitD, or left unactivated. d. Concentrations of indicated cytokines in culture supernatants of CD4 ${ }^{+} \mathrm{T}$ cells treated with escalating doses of $25(\mathrm{OH})$ VitD for $72 \mathrm{~h}$. e, IL6 mRNA, fold change compared to day 1 carrier (above), and IL-6 protein concentration in matched supernatants (below), at days 1, 3 and 5 in carrier and VitD-treated CD4 ${ }^{+}$T cell cultures. f, Representative flow cytometry plot (left) and cumulative data (right) of intracellular IL-6 expression in T cells treated with carrier or VitD (assay carried out on day 3). Cells gated based on lymphocyte gate (forward scatter, side scatter), singlets, live cells and CD4+ cells. $\mathbf{g}$, IL-10 concentrations in supernatants of CD4+ ${ }^{+}$cells cultured in the presence of increasing concentrations of IL- 6 for 72 hours. h, IL-17 concentrations in supernatants of CD4 ${ }^{+}$T cells cultured in the presence of increasing concentrations of IL-6, with and without VitD for 72 hours. i, Volcano plot representing changes in protein phosphorylation on phospho-kinase array comparing VitD-treated versus carrier treated cells. Data are from $n=2$ independent experiments. Thresholds for significance have been set at 1.2 fold change in phosphorylation in either direction at p-value $<0.05$. Please also see Fig. 3f,g. Marked are phosphoproteins that show significant changes in phosphorylation on VitD treatment. j, Quantification of pY-STAT3, STAT3, p-c-JUN, c-JUN and Hsp90 from immunoblots of lysates of CD4 ${ }^{+} T$ cells treated with carrier or VitD with, and without, Tocilizumab (Toc) at the concentrations shown. Bars show mean $+\operatorname{sem}$ from $n=3$ independent experiments. Please also see Fig. 3h. k-I, Shown are representative Western blots ( $\mathbf{k}$ ) and quantifications (I) of STAT3 and HSP90 in Carrier- and VitD-treated CD4+ $\mathrm{T}$ cells in the presence and absence of STAT3 siRNA. Data are representative of $n=2$ experiments carried out. Unless indicated, all cells in Supplementary Fig. 7 have been activated with $\alpha$-CD3 $+\alpha-C D 28$. Cumulative data in $\mathbf{b}, \mathbf{d}-\mathbf{h}, \mathbf{j}$ depict mean + sem. Unless indicated, all experiments have been carried out $n=3$ times. All statistical tests are two-sided. ${ }^{\star} p<0.05,{ }^{\star \star} p<0.01,{ }^{\star \star \star} p<0.001,{ }^{\star \star * \star} p<0.0001$ by one-way ANOVA $(\mathbf{d}, \mathbf{j})$, two-way ANOVA $(\mathbf{b}, \mathbf{e}, \mathbf{h})$ and paired t-test (f). Statistical comparisons in $\mathbf{d}$ and $\mathbf{h}$ compare VitD-treated (d) or IL-6-treated (h) cells against untreated cells. 
a
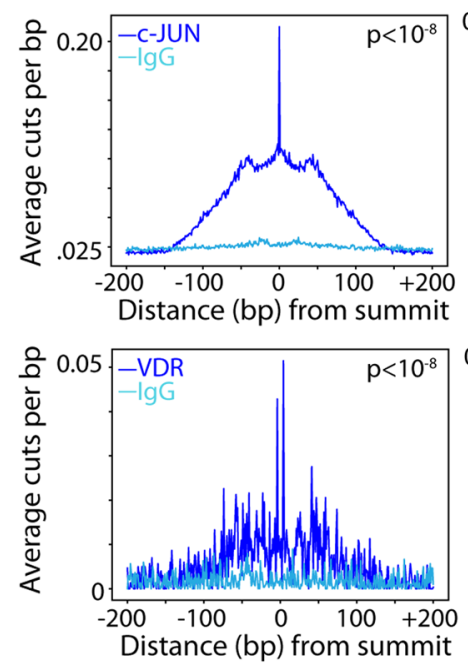

0.50
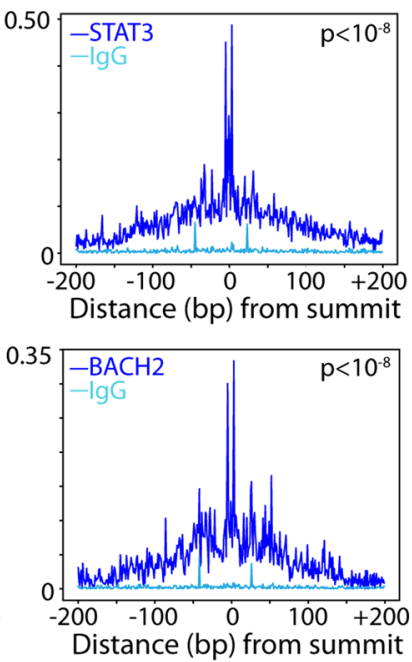

b

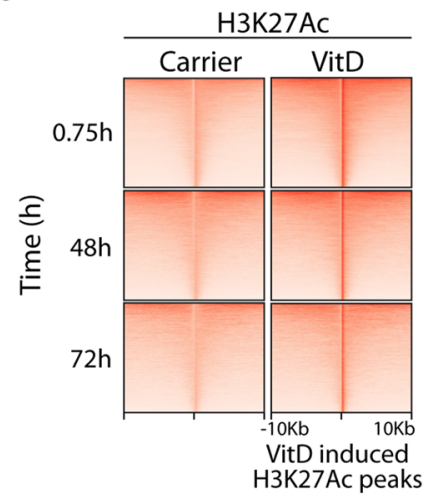

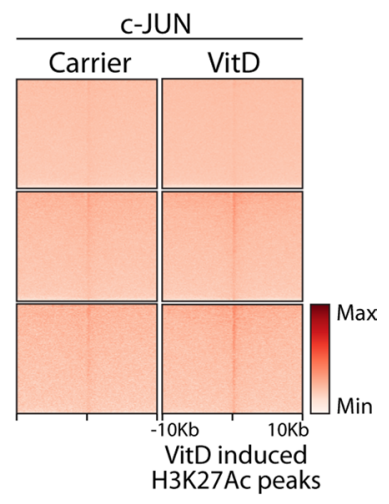

C

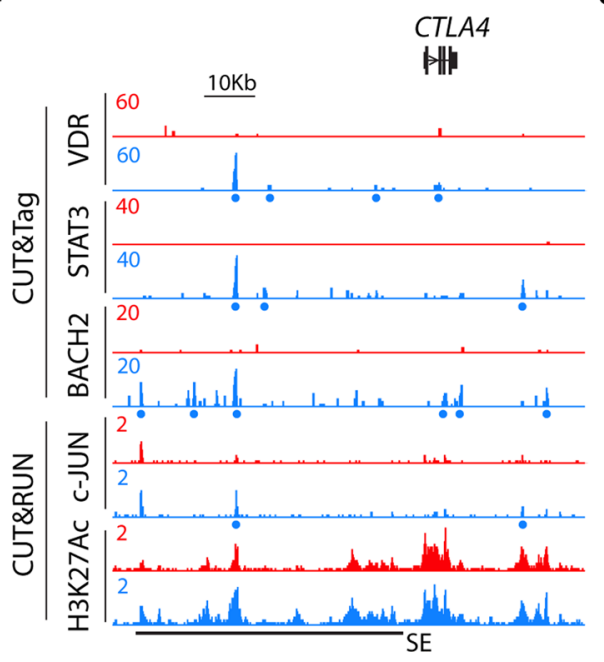

d

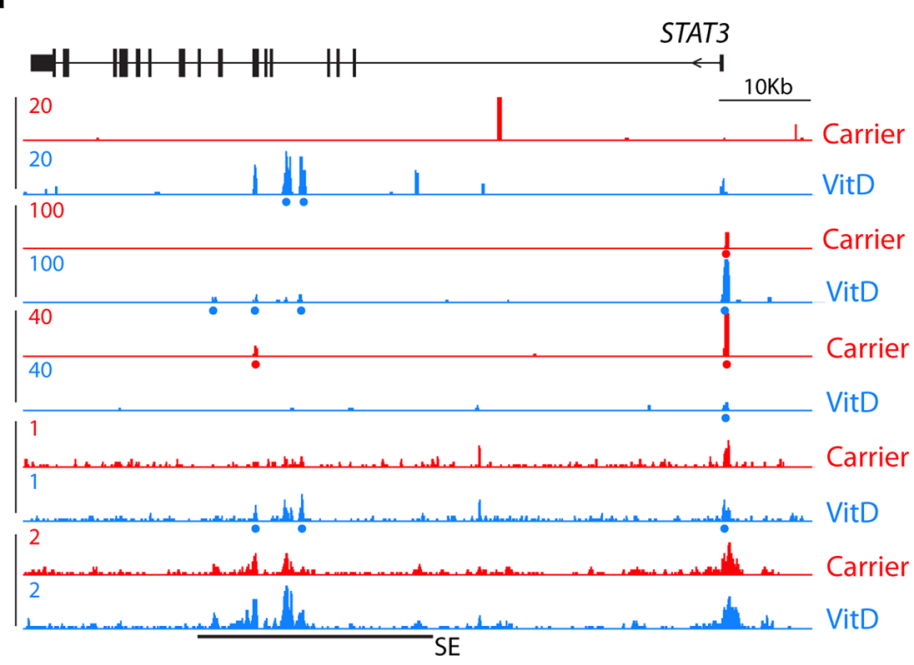

Extended Data Fig. 8 | Vitamin D recruits key transcription factors. a, histograms showing average cuts per bp in relation to the summits of c-JUN, STAT3, VDR and BACH2 peaks in VitD-treated CD4 ${ }^{+}$T cells. Shown are data from CUT\&RUN (c-JUN) and CUT\&Tag (VDR, STAT3 and BACH2) carried out with IgG (cyan) or antibodies specific to each TF (dark blue). p-values from K-S tests are indicated. b, heatmaps showing H3K27Ac and c-JUN signals at VitD-repressed and VitD-induced peaks over time. Time points are indicated. c-d, genome browser tracks at the CTLA4 (c) and STAT3 (d) loci showing H3K27Ac, c-JUN, BACH2, STAT3 and VDR binding in Carrier and VitD-treated cells. Red and blue dots represent peaks in Carrier and VitD-treated cells, respectively. SE denotes super-enhancer regions. Track heights are indicated on the left corner for each track. All cells in Supplementary Fig. 8 have been activated with $\alpha-C D 3+\alpha-C D 28$. 
a

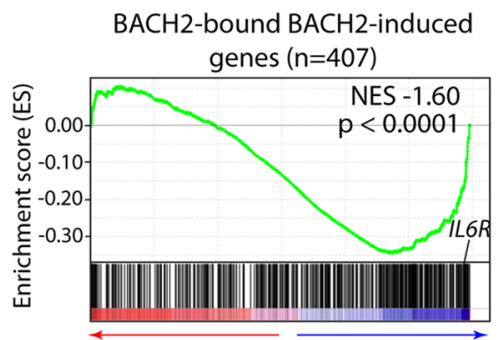

Higher in Carrier Higher in VitD

d

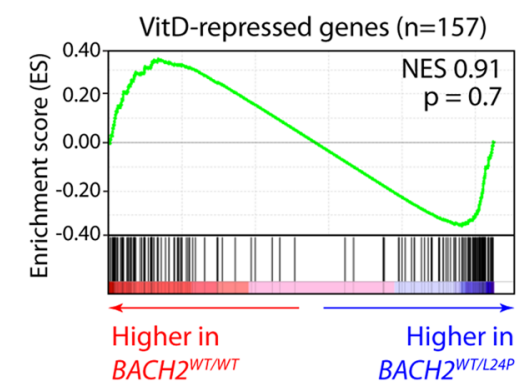

f

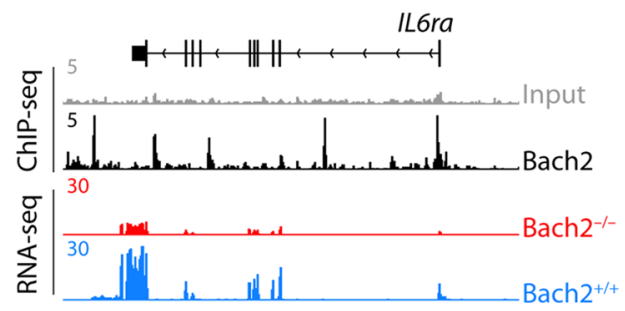

b

BACH2-bound BACH2-repressed genes $(n=691)$

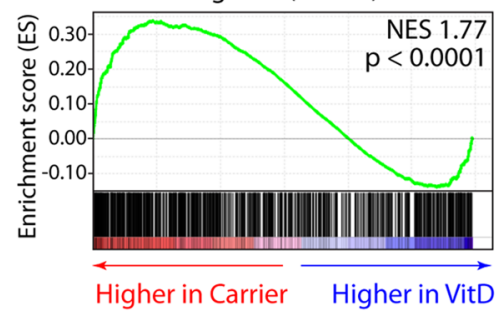

e

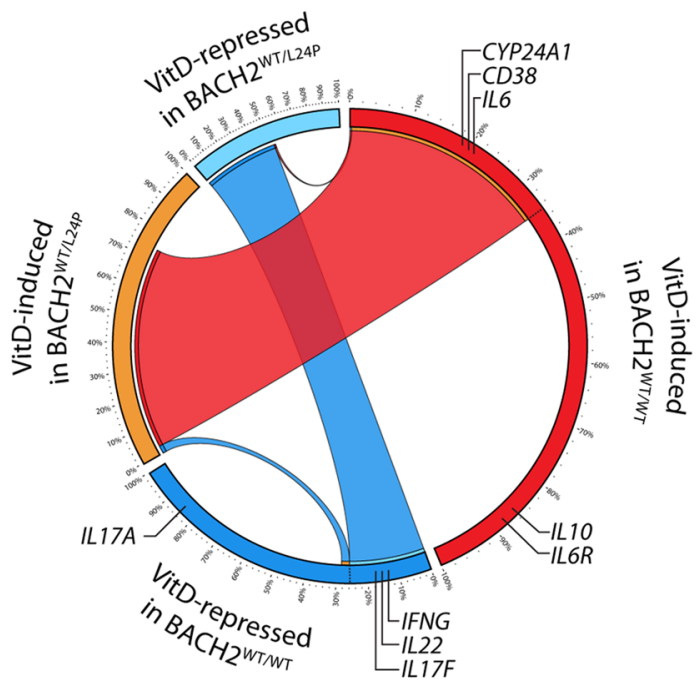

C

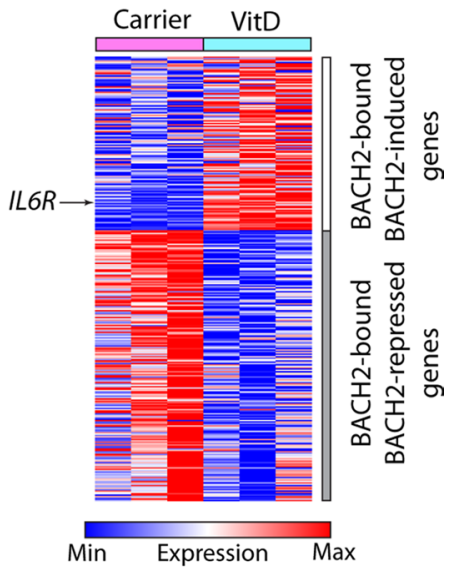

Extended Data Fig. 9 | A subset of Vitamin D-regulated genes are dependent on BACH2. a-c, GSEA comparing the transcriptomes of carrier and VitD-treated $\mathrm{CD}^{+} \mathrm{T}$ cells against BACH2-bound BACH2-induced genes (a) and -repressed genes $(\mathbf{b})$. Shown in $\mathbf{c}$ are the leading edges of the two GSEA enrichment plots in $\mathbf{a}-\mathbf{b}$. Marked in $\mathbf{a}$ and $\mathbf{c}$ is the IL-6 receptor (IL6R). d, GSEA comparing enrichment in VitD-repressed genes of the transcriptomes of VitD-treated $B A C H 2^{W T / W T}$ haplo-sufficient and BACH2 ${ }^{\text {WT/L24P }}$ haplo-insufficient $C D 4^{+} T$ cells. e, Circos diagram showing VitD-induced and repressed genes in VitD-treated BACH2 ${ }^{\text {WT/WT }}$ haplo-sufficient and BACH2 ${ }^{\text {WT/L24P }}$ haplo-insufficient $\mathrm{CD} 4^{+} \mathrm{T}$ cells. Cords join shared genes in patient and control. Indicated are the shared VitD-induced genes (CYP24A1, CD38 and IL6) and genes only induced by VitD in the presence of two wild-type copies of BACH2 (IL10 and IL6R).f, Genome browser tracks showing Bach2 ChIP-seq at the IL6ra locus and expression of IL6ra mRNA in CD4+ T cells of Bach2 wild-type (Bach2 ${ }^{+/+}$) and knock-out (Bach2-1-) mice. Track heights are indicated on the left corner for each track. Source data are from GSE45975. Empirical p-values are shown in $\mathbf{a}-\mathbf{b}$ and $\mathbf{d}$. NES $=$ normalized enrichment score. 
a

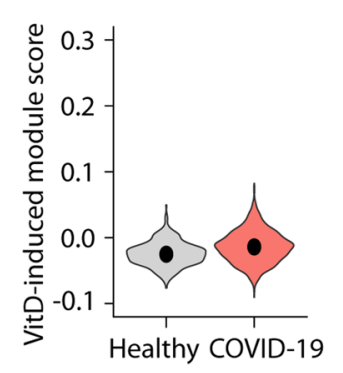

b

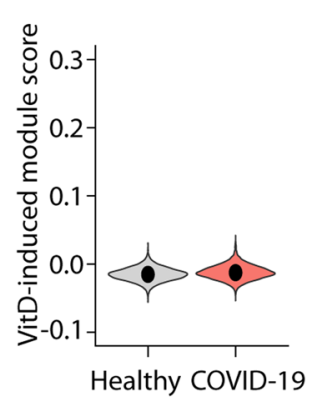

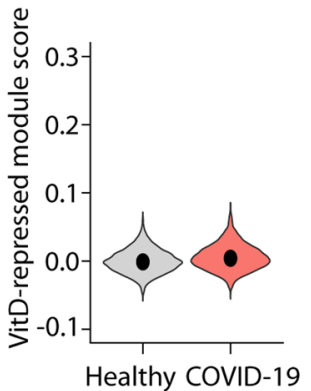

C

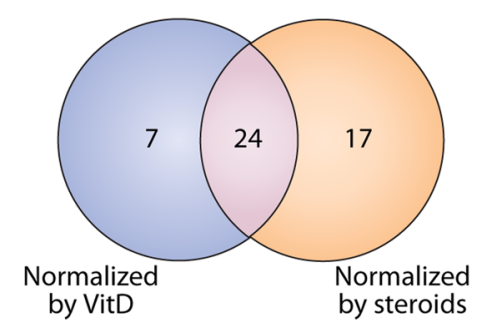

d

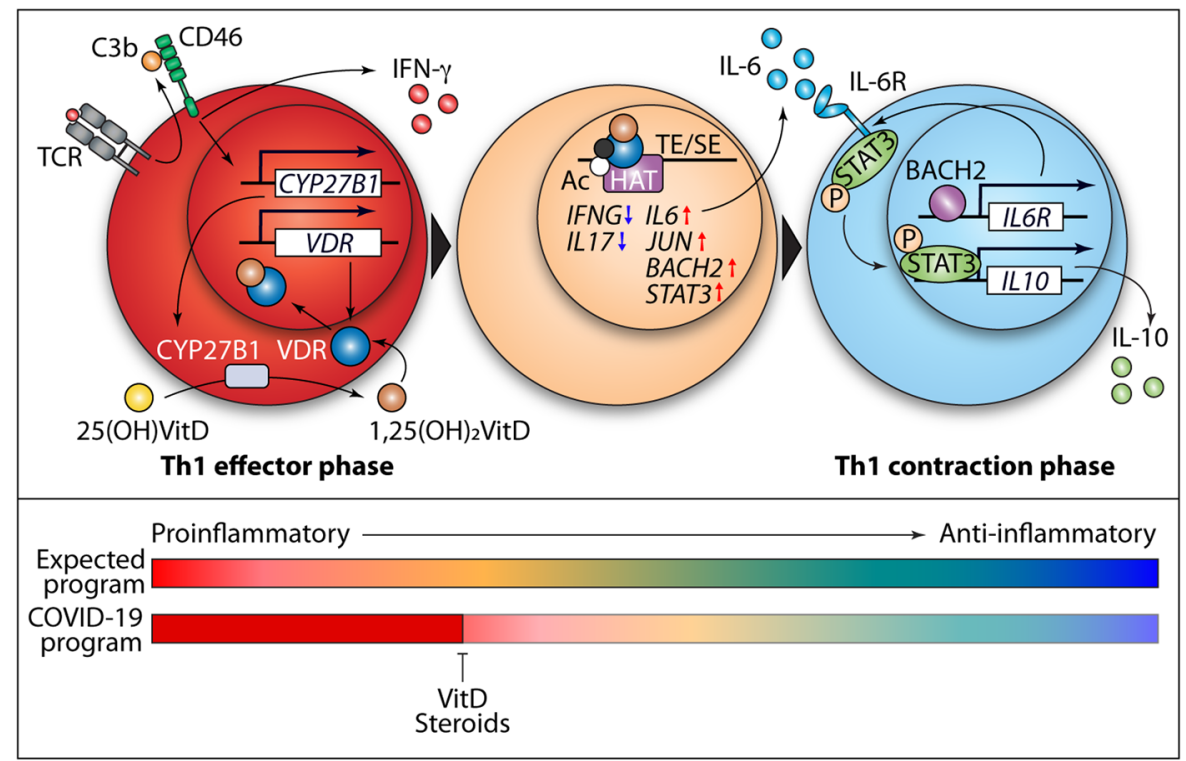

e

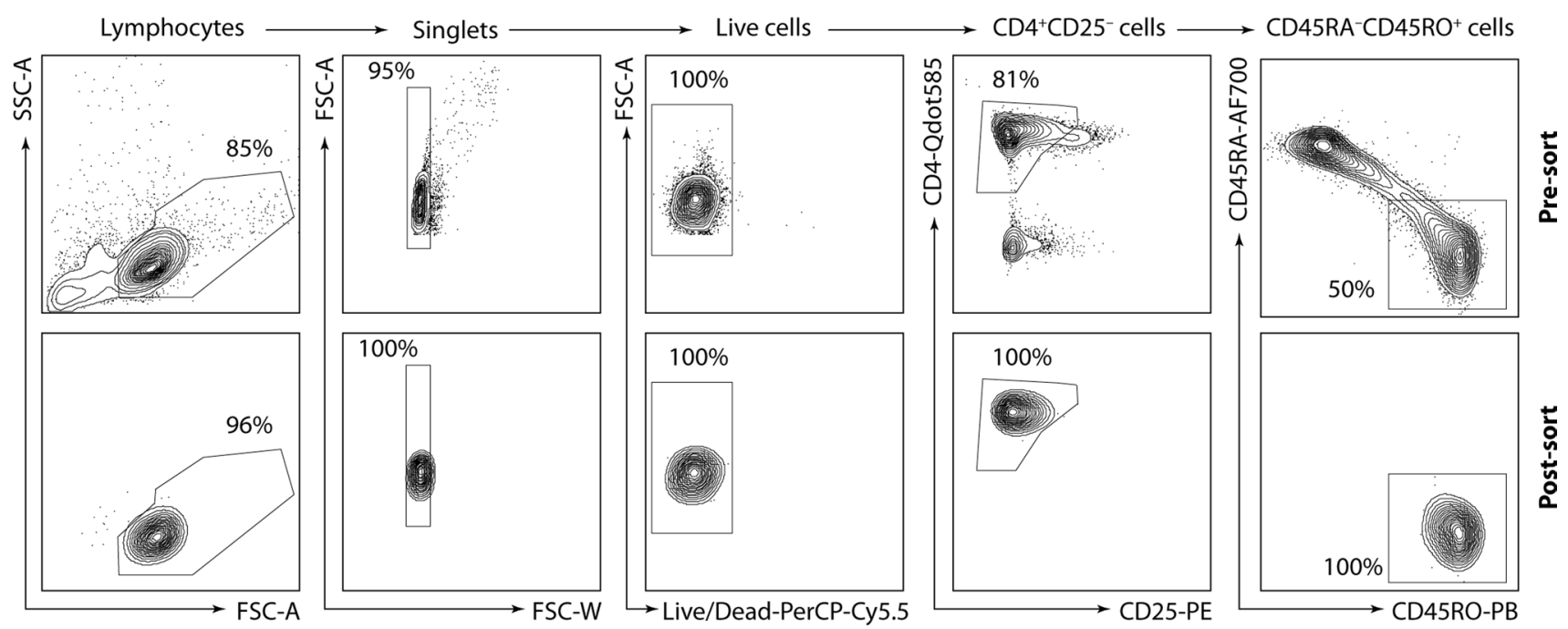

Extended Data Fig. 10 | See next page for caption. 
Extended Data Fig. 10 | Vitamin D-induced genes do not distinguish CD4+ BALF T cells of patients with COVID-19 from healthy controls. a, Violin plots showing expressions of VitD-induced genes, summarized as module scores, of BALF CD4+ ${ }^{+}$cells of patients with COVID-19 and healthy controls. Data are from $n=8$ patients with COVID-19 and $n=3$ healthy controls, sourced from GSE145926 and GSE122960. b, Violin plots showing expressions of VitD-induced and VitD-repressed genes, summarized as module scores, of PBMC CD4 ${ }^{+} T$ cells of patients with COVID-19 and healthy controls. Data are from $n=6$ patients with COVID-19 and $n=6$ healthy subjects, obtained from GSE150728. c, Venn diagram showing overlap between COVID-induced genes in CD4+ BALF T cells that are predicted to be normalized by VitD treatment versus those that are predicted to be normalized by steroid drugs. Please also see Supplementary Table 7. d. Schematic model of autocrine VitD-driven Th1 contraction program and potential intervention of impaired COVID-19 program with VitD and cortico-steroids. e, representative flow sorting strategy and example of post-sort purity obtained for isolation of memory CD4 ${ }^{+} \mathrm{T}$ cells in this paper. 


\section{nature portfolio}

Corresponding author(s): Behdad Afzali, Majid Kazemian

Last updated by author(s): Oct 17, 2021

\section{Reporting Summary}

Nature Portfolio wishes to improve the reproducibility of the work that we publish. This form provides structure for consistency and transparency in reporting. For further information on Nature Portfolio policies, see our Editorial Policies and the Editorial Policy Checklist.

\section{Statistics}

For all statistical analyses, confirm that the following items are present in the figure legend, table legend, main text, or Methods section.

n/a Confirmed

$\square$ The exact sample size $(n)$ for each experimental group/condition, given as a discrete number and unit of measurement

$\square$ \ A statement on whether measurements were taken from distinct samples or whether the same sample was measured repeatedly

$\square$ The statistical test(s) used AND whether they are one- or two-sided

Only common tests should be described solely by name; describe more complex techniques in the Methods section.

$\bigotimes$ A description of all covariates tested

$\square \bigotimes$ A description of any assumptions or corrections, such as tests of normality and adjustment for multiple comparisons

$\triangle$ A full description of the statistical parameters including central tendency (e.g. means) or other basic estimates (e.g. regression coefficient)

$\bigotimes$ AND variation (e.g. standard deviation) or associated estimates of uncertainty (e.g. confidence intervals)

$\varnothing$ For null hypothesis testing, the test statistic (e.g. $F, t, r$ ) with confidence intervals, effect sizes, degrees of freedom and $P$ value noted

Give $P$ values as exact values whenever suitable.

Х $\square$ For Bayesian analysis, information on the choice of priors and Markov chain Monte Carlo settings

Х $\square$ For hierarchical and complex designs, identification of the appropriate level for tests and full reporting of outcomes

$\bigotimes$ Estimates of effect sizes (e.g. Cohen's d, Pearson's $r$ ), indicating how they were calculated

Our web collection on statistics for biologists contains articles on many of the points above.

\section{Software and code}

Policy information about availability of computer code

Data collection Details have been provided in the methods

Data analysis Details have been provided in the methods

For manuscripts utilizing custom algorithms or software that are central to the research but not yet described in published literature, software must be made available to editors and reviewers. We strongly encourage code deposition in a community repository (e.g. GitHub). See the Nature Portfolio guidelines for submitting code \& software for further information.

\section{Data}

Policy information about availability of data

All manuscripts must include a data availability statement. This statement should provide the following information, where applicable:

- Accession codes, unique identifiers, or web links for publicly available datasets

- A description of any restrictions on data availability

- For clinical datasets or third party data, please ensure that the statement adheres to our policy 


\section{Field-specific reporting}

Please select the one below that is the best fit for your research. If you are not sure, read the appropriate sections before making your selection. $\bigotimes$ Life sciences $\quad \square$ Behavioural \& social sciences $\quad \square$ Ecological, evolutionary \& environmental sciences

For a reference copy of the document with all sections, see nature.com/documents/nr-reporting-summary-flat.pdf

\section{Life sciences study design}

All studies must disclose on these points even when the disclosure is negative.

Sample size

No mice were used in this manuscript. Patient samples were limited by availability, so no sample size calculations were performed beforehand. For in vitro experiments, we typically use 3 to 7 samples, which, based on our experience gives enough statistical power for these in vitro experiments.

Data exclusions No data have been excluded

Replication To ensure reproducibility, all experiments have been replicated a minimum of two times. RNA-seq using cells from the BACH2 haploinsufficient patient was done once as the $\mathrm{BACH} 2$ patient is a very rare patient that is currently unwell and unable to donate blood a second time.

Randomization Random allocation was used.

Blinding Investigators were blinded to treatment group when analyzing BACH2 staining in dermal specimens. For other experimental procedures, for example Western blotting, blinding is not practical since the order of data acquisition/sample loading is important for presentation.

\section{Reporting for specific materials, systems and methods}

We require information from authors about some types of materials, experimental systems and methods used in many studies. Here, indicate whether each material, system or method listed is relevant to your study. If you are not sure if a list item applies to your research, read the appropriate section before selecting a response.

Materials \& experimental systems

\begin{tabular}{l|l}
\hline$n / a$ & Involved in the study \\
\hline & $\bigotimes$ Antibodies \\
$\square$ & $\square$ Eukaryotic cell lines \\
$\square$ & $\square$ Aniaeontology and archaeology \\
$\square$ & $\square$ Clinical data \\
$\square$ Dual use research of concern
\end{tabular}

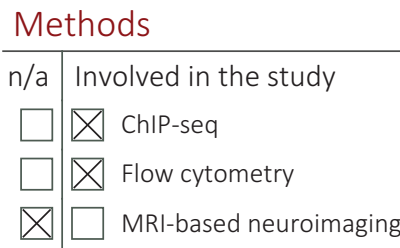

\section{Antibodies}

Antibodies used

All antibodies were validated by the manufacturer. Manufacture details provided on respective company websites include below. No specific lot numbers were noted.

$\operatorname{VDR}(\mathrm{D}-6$, Santa Cruz),

pSTAT3 (D3A7, Cell Signaling Technologies),

STAT3 (124H6, Cell Signaling Technologies),

Phospho-c-Jun (S63, R\&D systems)

c-Jun (L70B11, Cell Signaling Technologies)

H3K27Ac (ab4729, Abcam),

c-JUN (60AB, Cell Signaling Technologies),

$\operatorname{lgG}(31235$, Thermo Fisher Scientific)

VDR (D2K6W, Cell Signaling Technologies)

STAT3 (D3Z2G, Cell Signaling Technologies)

BACH2 (D3T3G, Cell Signaling Technologies)

Validation

VDR (D-6, Santa Cruz),

VDR (D-6) is recommended for detection of VDR of mouse, rat and human origin by Western Blotting (starting dilution 1:100, dilution range 1:100-1:500), immunoprecipitation [1-2 $\mu \mathrm{g}$ per 100-500 $\mu \mathrm{g}$ of total protein (1 ml of cell lysate)], immunofluorescence (starting dilution 1:50, dilution range 1:50-1:500) and immunohistochemistry (including paraffin-embedded sections) (starting dilution 1:50, 
dilution range 1:50-1:500). Suitable for use as control antibody for VDR siRNA (h): sc-106692, VDR siRNA (m): sc-36811, VDR shRNA Plasmid (h): sc-106692-SH, VDR shRNA Plasmid (m): sc-36811-SH, VDR shRNA (h) Lentiviral Particles: sc-106692-V and VDR shRNA (m) Lentiviral Particles: sc-36811-V. VDR (D-6) X TransCruz antibody is recommended for Gel Supershift and ChIP applications. Molecular Weight (predicted) of VDR isoforms: 48/53 kDa. Molecular Weight (observed) of VDR isoforms: 48-60 kDa. Positive Controls: HeLa whole cell lysate: sc-2200, MCF7 whole cell lysate: sc-2206 or SK-BR-3 cell lysate: sc-2218.

VDR (D-6) is a mouse monoclonal antibody raised against amino acids 344-424 of VDR of human origin.

Manufacturer provides validation by Western and immunohistochemistry.

pSTAT3 (D3A7, Cell Signaling Technologies),

Species Reactivity:

Human, Mouse, Rat, Monkey

Monoclonal antibody is produced by immunizing animals with a synthetic phosphopeptide corresponding to residues surrounding Tyr705 of mouse Stat3.

Phospho-Stat3 (Tyr705) (D3A7) XP ${ }^{\circledR}$ Rabbit mAb detects endogenous levels of Stat3 only when phosphorylated at tyrosine 705. This antibody does not cross-react with phospho-EGFR or the corresponding phospho-tyrosines of other Stat proteins.

Manufacturer provides validation by Western, IP, IHC, IF, Fluor, and ChIP

STAT3 (124H6, Cell Signaling Technologies),

Specificity / Sensitivity

Stat3 (124H6) Mouse mAb detects endogenous levels of total Stat3 protein.

Species Reactivity:

Human, Mouse, Rat, Monkey

Source / Purification

Monoclonal antibody is produced by immunizing animals with a synthetic peptide centered around amino acid Gln692 of human Stat3.

Manufacturer provides validation by Western, IP, IHC, IF, FACS, and ChIP.

Phospho-c-Jun (S63, R\&D systems)

Species Reactivity

Human

Specificity

Detects human c-Jun when phosphorylated at S63 in Western blots.

Source

Recombinant Monoclonal Rabbit IgG Clone \# 1018Y

Purification

Protein A or $\mathrm{G}$ purified from cell culture supernatant

Immunogen

Phosphopeptide containing the human c-Jun S63 site

Accession \# P05412

Formulation

Lyophilized from a $0.2 \mu \mathrm{m}$ filtered solution in PBS with Trehalose. *Small pack size (SP) is supplied as a $0.2 \mu \mathrm{m}$ filtered solution in PBS Label

Unconjugated

Manufacturer provides validation by Western and immunofluorescence

c-Jun (L70B11, Cell Signaling Technologies)

Product Usage Information

Specificity / Sensitivity

c-Jun (L70B11) Mouse mAb detects endogenous levels of total c-Jun protein, regardless of phosphorylation state.

Species Reactivity:

Human, Mouse, Rat, Monkey

Source / Purification

Monoclonal antibody is produced by immunizing animals with a recombinant fragment of c-Jun protein.

Manufacturer provides validation by Western blotting.

H3K27Ac (ab4729, Abcam)

Description

Rabbit polyclonal to Histone H3 (acetyl K27) - ChIP Grade

Host species

Rabbit

Tested applications

Suitable for: ICC/IF, WB, IHC-P, ChIP, PepArrmore details

Species reactivity

Reacts with: Mouse, Cow, Human, Recombinant fragment

Predicted to work with: Rat, Chicken, Xenopus laevis, Arabidopsis thaliana, Drosophila melanogaster, Monkey, Zebrafish, Plasmodium falciparum, Rice, Cyanidioschyzon merolae

Immunogen

Synthetic peptide corresponding to Human Histone H3 aa 1-100 (acetyl K27) conjugated to keyhole limpet haemocyanin

(Peptide available as ab24404) 
c-JUN (60AB, Cell Signaling Technologies),

Specificity / Sensitivity

c-Jun (60A8) Rabbit mAb detects endogenous levels of total c-Jun protein, regardless of phosphorylation state.

Species Reactivity:

Human, Mouse, Rat, Monkey

Source / Purification

Monoclonal antibody is produced by immunizing animals with a GST-c-Jun protein corresponding to the amino-terminal sequence of human c-Jun.

Manufacturer provides validation by Western, IP, IHC, IF, Facs, and ChIP.

$\operatorname{lgG}$ (31235, Thermo Fisher Scientific)

Purity: Based on immunoelectrophoresis at an antigen concentration of $20 \mathrm{mg} / \mathrm{mL}$, the pattern of precipitation against goat antirabbit whole serum is the same as that against goat anti-rabbit IgG, Fc fragment specific. This antibody is recommended for use in blocking assays, immunohistochemical, immunocytochemical and immunofluorescent applications. Store product at 2- $8^{\circ} \mathrm{C}$ until opened. After opening dilute only enough product for a single day's use. Store remainder at $2-8^{\circ} \mathrm{C}$ under sterile conditions. Country of Origin: USA

Manufacturer provides validation of isotype by flow cytometry.

VDR (D2K6W, Cell Signaling Technologies)

Specificity / Sensitivity

Vitamin D3 Receptor (D2K6W) Rabbit mAb recognizes endogenous levels of total vitamin D3 receptor protein. This antibody does not cross-react with vitamin D3 receptor-like proteins. Based upon sequence alignment, this antibody is predicted to react with both VDRB1 and VDRB2 isoforms.

Species Reactivity:

Human, Mouse

Species predicted to react based on $100 \%$ sequence homology:

Hamster, Bovine, Pig, Horse

Source / Purification

Monoclonal antibody is produced by immunizing animals with a synthetic peptide corresponding to residues near the amino terminus of human vitamin D3 receptor isoform A protein.

Manufacturer provides validation by Western, IP, IHC, and ChIP.

STAT3 (D3Z2G, Cell Signaling Technologies)

Specificity / Sensitivity

Stat3 (D3Z2G) Rabbit mAb recognizes endogenous levels of total Stat3 protein. Species cross-reactivity for IF-IC and F is human only. Species Reactivity:

Human, Mouse, Rat, Monkey

Species predicted to react based on $100 \%$ sequence homology:

Bovine, Pig

Source / Purification

Monoclonal antibody is produced by immunizing animals with a synthetic peptide corresponding to residues surrounding Gly 700 of human Stat3 protein.

Manufacturer provides validation by Western, IP, IF, FACS, and ChIP

STAT3 (D3Z2G, Cell Signaling Technologies)

Specificity / Sensitivity

Stat3 (D3Z2G) Rabbit mAb recognizes endogenous levels of total Stat3 protein. Species cross-reactivity for IF-IC and F is human only Species Reactivity:

Human, Mouse, Rat, Monkey

Species predicted to react based on $100 \%$ sequence homology:

Bovine, Pig

Source / Purification

Monoclonal antibody is produced by immunizing animals with a synthetic peptide corresponding to residues surrounding Gly 700 of human Stat3 protein.

Manufacturer provides validation by western, IP, IF, FACS, and ChIP.

BACH2 (D3T3G, Cell Signaling Technologies)

Specificity / Sensitivity

BACH2 (D3T3G) Rabbit mAb recognizes endogenous levels of total BACH2 protein.

Species Reactivity:

Human

Source / Purification

Monoclonal antibody is produced by immunizing animals with a synthetic peptide corresponding to residues surrounding Ala304 of human $\mathrm{BACH} 2$ protein.

Manufacturer provides validation by Western, IP, and ChIP 
Policy information about studies involving human research participants

Population characteristics

Patients were recruited, together with matched controls, for the following clinical phenotypes: hyperlgE syndrome, CD46 deficiency, psoriasis or BACH2 haploinsufficiency

HyperlgE syndrome patients - 1 female aged 22, 2 males aged 28 and 36

CD46 deficient patients -1 female aged 32, 2 males aged 22 and 29

Psoriasis patients -3 females aged 52, 45 and 56, 2 males aged 32 and 35

BACH2WT/L24P patient - female aged 26

Recruitment

Patients with HyperlgE syndrome were recruited through their physicians or by self-referral on to a natural history of HyperlgE syndrome protocol. The protocol has enrolled a diverse ethnic, racial and socioeconomic cohort in order to avoid bias by offering enrollment to anyone with a dominant negative STAT3 mutation.

BACH2WT/L24 patient - she is the only subject identified in the world with the L24P mutation, which is the most severe form of BACH2 haploinsufficiency we have described in peer-reviewed work so far (Afzali et al., Nat Immunol 2017). She was referred to our service at the age of 18 year with a hyperinflammatory condition for tertiary care management. Due to her early onset of disease (initial symptoms as an infant); the severity of her symptoms; and the particular combinations of symptoms we arranged trio exome sequencing to identify any abnormal de novo genes. Other recessive genes were ruled out; the $\mathrm{BACH} 2$ gene mutation was considered potentially damaging, hence further investigation was pursued and published subsequently

Patients with psoriasis were recruited by self-referral or by referral by their physicians into our natural history study. This protocol enrolls a diverse ethnic, racial and socioeconomic cohort in order to avoid bias by offering enrollment to anyone with lesional psoriasis over the age of 18

Adult patients with CD46 deficiencies were recruited under appropriate institutional guidelines for investigation of the cause of atypical hemolytic uremic syndrome and were found on sequencing to have mutations in CD46. The cases have been previously described (Fremeaux-Bacchi et al, J Am Soc Nephrol 2006; Couzi et al., Am J Kidney Dis 2008). There was no ethnic, racial and socioeconomic bias in recruitment of subjects.

Ethics oversight

Human studies were conducted in accordance with the Declaration of Helsinki and approved by the Institutional Review Board of Guy's Hospital (reference 09/H0707/86), National Institutes of Health (approval numbers 7458, PACl, 13-H-0065 and 00-1-0159) and Imperial College London (approval number 12/WA/0196 ICHTB HTA license number 12275 to project RI4098). All patients provided informed written consent.

Note that full information on the approval of the study protocol must also be provided in the manuscript.

\section{ChIP-seg}

\section{Data deposition}

$\bigotimes$ Confirm that both raw and final processed data have been deposited in a public database such as GEO.

$\bigotimes$ Confirm that you have deposited or provided access to graph files (e.g. BED files) for the called peaks.

Data access links

May remain private before publication.

Files in database submission

Genome browser session

(e.g. $\underline{\operatorname{UCSC}}$

Data generated for this study are deposited at the Gene Expression Omnibus (GEO) under GSE154741.

Please see above

N/A

\section{Methodology}

Replicates

Sequencing depth

CUT\&RUN were repeated once ( $n$ of 1 shown and deposited)

CUT\&Tag were carried out once for each target protein

Antibodies

For CUT\&RUN: 15-25million reads/CUT\&RUN

For CUT\&Tag: 1-3million reads/CUT\&Tag

H3K27Ac (ab4729, Abcam)

c-JUN (60AB, Cell Signaling Technologies),

IgG (31235, Thermo Fisher Scientific)

VDR (D2K6W, Cell Signaling Technologies)

STAT3 (D3Z2G, Cell Signaling Technologies)

BACH2 (D3T3G, Cell Signaling Technologies)

Peak calling parameters H3K27Ac CUT\&RUN: MACS2: with parameter '-f BAMPE --nomodel'.

JUN CUT\&RUN: MACS2: '--nomodel -g hs -f BAMPE -q 0.01 --SPMR --keep-dup all'. 
Data quality

Software

For CUT\&RUN and CUT\&Tag, default HOMER settings were used, which includes a default $\mathrm{p}$-value threshold $<0.05$ for motif enrichment. We then filtered out Hg19 Blacklist regions and performed motifs enrichment analysis on the remaining HOMERidentified peaks (findMotifsGenome.pl). The top motifs identified corresponded to the transcription factors that were assayed.

As detailed in the methods, our data analysis pipeline for CUT\&RUN and Tag data utilized the following software:

Trimmomatic: Trimmomatic is a fast, multithreaded command line tool that can be used to trim and crop Illumna (FASTQ) data as well as to remove adapters. These adapters can pose a real problem depending on the library preparation and downstream application. There are two major modes of the program: Paired end mode and Single end mode. The paired end mode will maintain correspondence of read pairs and also use the additional information contained in paired reads to better find adapter or PCR primer fragments introduced by the library preparation process. Trimmomatic works with FASTQ files (using phred +33 or phred +64 quality scores, depending on the Illumina pipeline used). Files compressed using either "gzip " or „bzip2" are supported, and are identified by use of „.gz" or „..bz2" file extensions.

Bowtie2: is an ultrafast and memory-efficient tool for aligning sequencing reads to long reference sequences. It is particularly good at aligning reads of about 50 up to 100s or 1,000s of characters, and particularly good at aligning to relatively long (e.g. mammalian) genomes. Bowtie 2 indexes the genome with an FM Index to keep its memory footprint small: for the human genome, its memory footprint is typically around 3.2 GB. Bowtie 2 supports gapped, local, and paired-end alignment modes.

MACS2: Captures the influence of genome complexity to evaluate the significance of enriched ChIP regions and MACS2 improves the spatial resolution of binding sites through combining the information of both sequencing tag position and orientation. MACS2 can be easily used for ChIP-Seq data alone, or with a control sample with the increase of specificity. Moreover, as a general peak-caller, MACS can also be applied to any "DNA enrichment assays" if the question to be asked is simply: where we can find significant reads coverage than the random background.

SEACR: SEACR is intended to call peaks and enriched regions from sparse CUT\&RUN or chromatin profiling data in which background is dominated by "zeroes" (i.e. regions with no read coverage). It requires R (https://www.r-project.org) and Bedtools (https:// bedtools.readthedocs.io/en/latest/) to be available in your path, and it requires bedgraphs from paired-end sequencing as input, which can be generated from read pair BED files (i.e. BED coordinates reflecting the $5^{\prime}$ and 3 ' termini of each read pair) using bedtools genomecov with the "-bg" flag, or alternatively from name-sorted paired-end BAM files. A description of the method can be found in the following manuscript. Meers MP, Tenenbaum D, Henikoff S. (2019). Peak calling by Sparse Enrichment Analysis for CUT\&RUN chromatin profiling. Epigenetics and Chromatin 12(1):42.

HOMER: HOMER (Hypergeometric Optimization of Motif EnRichment) is a suite of tools for Motif Discovery and next-gen sequencing analysis. It is a collection of command line programs for UNIX-style operating systems written in Perl and C++. HOMER was primarily written as a de novo motif discovery algorithm and is well suited for finding 8-20 bp motifs in large scale genomics data. HOMER contains many useful tools for analyzing ChIP-Seq, GRO-Seq, RNA-Seq, DNase-Seq, Hi-C and numerous other types of functional genomics sequencing data sets.

Integrative Genomics Viewer (IGV): The Integrative Genomics Viewer (IGV) is a high-performance, easy-to-use, interactive tool for the visual exploration of genomic data. It supports flexible integration of all the common types of genomic data and metadata, investigator-generated or publicly available, loaded from local or cloud sources.

deepTools is a suite of python tools particularly developed for the efficient analysis of high-throughput sequencing data, such as ChIP-seq, RNA-seq or MNase-seq. This software is primarly utilized to normalize signal data and to produce comparative histograms and heatmaps.

\section{Flow Cytometry}

Plots

Confirm that:

$\bigotimes$ The axis labels state the marker and fluorochrome used (e.g. CD4-FITC).

$\bigotimes$ The axis scales are clearly visible. Include numbers along axes only for bottom left plot of group (a 'group' is an analysis of identical markers).

$\bigotimes$ All plots are contour plots with outliers or pseudocolor plots.

$\triangle$ A numerical value for number of cells or percentage (with statistics) is provided.

\section{Methodology}

Sample preparation

Cells were stained $30 \mathrm{~min}$ at $4 \mathrm{C}$ in a final volume of $100 \mathrm{mcL}$ staining buffer with antibodies against CD4 (OKT4, Thermo Fisher Scientific), IL-6 (MQ2-13A5, BioLegend), LAG-3 (3DS223H, Thermo Fisher Scientific), CD49b (P1H5, Thermo Fisher Scientific) with DAPI or LIVE/DEAD Fixable Aqua/Violet (Invitrogen) to exclude dead cells. For intracellular staining, cells were treated with the spiked addition of Phorbol 12-myristate 13-acetate (PMA) $(50 \mathrm{ng} / \mathrm{mL}$, Sigma-Aldrich), ionomycin $(1 \mathrm{mcg} / \mathrm{mL}$, SigmaAldrich), GolgiStop (1X, BD Biosciences) and Brefeldin A (1X, BD Biosciences) to media then cultured 5h, subsequently washed in PBS, surface stained, treated with Cytofix/Cytoperm (eBioscience) and subsequent Perm/Wash buffer (eBioscience) steps before incubation with intracellular antibodies for $30 \mathrm{~min}$ at $4 \mathrm{C}$. Samples were washed and acquired on a LSRFortessa (BD Biosciences) or the Attune NxT Flow Cytometer (Invitrogen) within 24h and analyzed using FlowJo/v9.9.6. 


\section{Software}

Cell population abundance

Gating strategy
Please see above

Post-sort purities were routinely over $99 \%$ and were tested by flow cytometry on a small number of sorted cells

Gating strategies are shown in the supplementary information.

Wick this box to confirm that a figure exemplifying the gating strategy is provided in the Supplementary Information. 